\title{
Explosive basaltic volcanism of the Chikurachki Volcano (Kurile arc, Russia): Insights on pre-eruptive magmatic conditions and volatile budget revealed from phenocryst-hosted melt inclusions and groundmass glasses
}

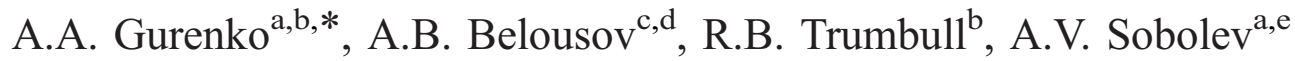 \\ ${ }^{a}$ Max Planck Institute for Chemistry, Geochemistry, P.O. Box 3060, 55020 Mainz, Germany \\ ${ }^{\mathrm{b}}$ GeoForschungsZentrum Potsdam, Section 4.2, Telegrafenberg, 14473 Potsdam, Germany \\ ${ }^{\mathrm{c} I n s t i t u t e}$ of Marine Geology and Geophysics, 693022 Yuzno-Sakhalinsk, Russia \\ ${ }^{\mathrm{d}}$ Institute of Volcanic Geology and Geochemistry, 683006 Petropavlovsk-Kamchatsky, Russia \\ ${ }^{\mathrm{e}}$ Vernadsky Institute of Geochemistry and Analytical Chemistry, 117975 Moscow, Russia
}

Received 13 December 2004; accepted 6 April 2005

\begin{abstract}
Highly explosive eruptions of basaltic composition are relatively rare and poorly understood, yet they may be quite important in terms of atmospheric impact because of the generally much higher concentrations of $\mathrm{S}$ in basaltic systems compared with the typical explosive felsic eruptions. We have determined concentrations of $\mathrm{H}_{2} \mathrm{O}$, major, trace and volatile $(\mathrm{S}, \mathrm{Cl})$ elements by EPMA and SIMS from melt inclusions and groundmass glasses of the 1986, 1853 and prehistoric explosive eruptions of basaltic magmas from the Chikurachki volcano, northern Kurile arc. Melt inclusions are hosted by olivine $\left(\mathrm{Fo}_{72-78}\right)$, orthopyroxene (mg\#=72-75), clinopyroxene $(\mathrm{mg} \#=71-77)$ and plagioclase $\left(\mathrm{An}_{74-96}\right)$ phenocrysts. Estimated crystallization conditions were in the range from 910 to $1180{ }^{\circ} \mathrm{C}$ at less than $400 \mathrm{MPa}$ total pressure and oxygen fugacity of NNO +1 to $+2 \log$ units. Inclusion glasses are basaltic to andesitic in composition. Relative to N-MORB, the melts are enriched in LREE ([La/ $\left.\mathrm{Sm}]_{n}=1.8-2.6\right)$ and $\mathrm{Sr}\left([\mathrm{Sr} / \mathrm{Ce}]_{n}=1.7-2.4\right)$, and show $\mathrm{Nb}, \mathrm{Zr}$ and Ti depletions typical for arc magmas $\left([\mathrm{Nb} / \mathrm{La}]_{n}=0.2-0.3\right.$, $[\mathrm{Zr} / \mathrm{Sm}]_{n}=0.6-0.9,[\mathrm{Ti} / \mathrm{Gd}]_{n}=0.4-1.1$ ). The pre-erupted melts were volatile rich (up to $6.4 \mathrm{wt} . \% \mathrm{H}_{2} \mathrm{O}, 2900 \mathrm{ppm} \mathrm{S}$ and 1400 $\mathrm{ppm} \mathrm{Cl}$ ), which may explain the strongly explosive character of volcanic activity. Much lower volatile contents in the groundmass glasses compared to inclusions reflect the extent of eruptive magma degassing. The wide range of volatile contents in glass inclusions is interpreted as a result of complex patterns of magma ascent, phenocryst growth and partial degassing at high-level reservoirs before eruption. The most $\mathrm{H}_{2} \mathrm{O}$ - and S-poor inclusions are close to groundmass values and may relate to magmas degassed in the summit reservoir, which drained back and mixed with less degassed magmas at depth. Estimations of total volatile release to the atmosphere, based on the difference between maximum melt inclusion contents and matrix glass, suggest that the 1986 and 1853 explosive eruptions released 3400 and 2500 metric tons $\mathrm{H}_{2} \mathrm{O}, 300$ and 200 tons $\mathrm{SO}_{2}$ and 8 and
\end{abstract}

\footnotetext{
* Corresponding author. Present address: Max-Planck-Institut für Chemie, Abteilung Geochemie, Postfach 3060, D-55020 Mainz, Germany. Tel.: +49 6131305 304; fax: +496131371051.

E-mail address: agurenko@mpch-mainz.mpg.de (A.A. Gurenko).
} 
12 tons $\mathrm{Cl}$, respectively. This is about ten times higher for $\mathrm{SO}_{2}$ and ten times lower for $\mathrm{Cl}$ than for the 1989-1990 eruptions of the Redoubt volcano.

(C) 2005 Elsevier B.V. All rights reserved.

Keywords: subduction zone; basaltic plinian eruptions; melt inclusions; degassing; Kurile arc; Chikurachki volcano

\section{Introduction}

Highly explosive volcanic (plinian) eruptions are characterized by very large eruption rates, high eruption columns and widely dispersed fallout deposits (Walker, 1973, 1980; Wilson and Walker, 1987). They therefore represent efficient mechanisms to transport volatiles and other material from Earth's interior to the upper atmosphere, where global dispersion can take place. Injection of water, halogens and sulfur into the atmosphere leads to formation of solid and liquid aerosols which can significantly affect global climate and the stability of the ozone layer (Brasseur and Granier, 1992). A number of factors influence the character of volcanic eruptions, including conduit geometry, magma viscosity and degassing history (e.g. Carey and Sparks, 1986). Typically, plinian eruptions are associated with water-rich intermediate to silicic magmas (andesitic to dacitic) and these have been the most thoroughly studied. Less common and much less well understood, are the highly explosive eruptions of basaltic magmas. Only a limited number of these are presently known in the literature and most of them are from subduction settings where water can be expected to play a key role. These are the plinian and subplinian eruptions of Tolbachik, Kamchatka (Fedotov and Markhinin, 1983); Masaya and Cerro Negro, Nicaragua (Williams, 1983; Roggensack et al., 1997); Sumisu Rift, Japan (Gill et al., 1990); Ambrym, Vanuatu (Robin et al., 1993); Tarawera 1886 eruption, New Zealand and Etna 122 BC, Italy (Houghton et al., 2004); and Chikurachki, Kurile Islands (Ovsyannikov and Muravyev, 1992; Belousov et al., 2003), which is the subject of our study.

The Chikurachki volcano is one of the three most active volcanoes of the Kurile arc, and it has a history of explosive eruptions of basaltic magma (Belousov et al., 2003). We present here new petrologic and geochemical data for phenocryst-hosted melt inclusions and groundmass glasses collected from plinian eruptive events of Chikurachki with the aim of determin- ing the pre-eruptive physical conditions of magma storage and the composition of the melt phase, particularly its water and volatile concentrations $(\mathrm{S}, \mathrm{Cl})$. From these data, we constrain the processes of magma evolution (crystallization, mixing and degassing) in the Chikurachki system, discuss the degassing behavior of the different volatile components, and estimate the release of water, sulfur dioxide and chlorine to the atmosphere from the eruptive events.

\section{Geological setting and eruptive styles}

Chikurachki is a young, very active stratovolcano located in the southern part of Paramushir Island in the northern Kurile island arc just south of Kamchatka (Fig. 1). Volcanism results from westward subduction of the Pacific plate under the Eurasian plate. The Late Holocene stratocone of the volcano is built on a thick

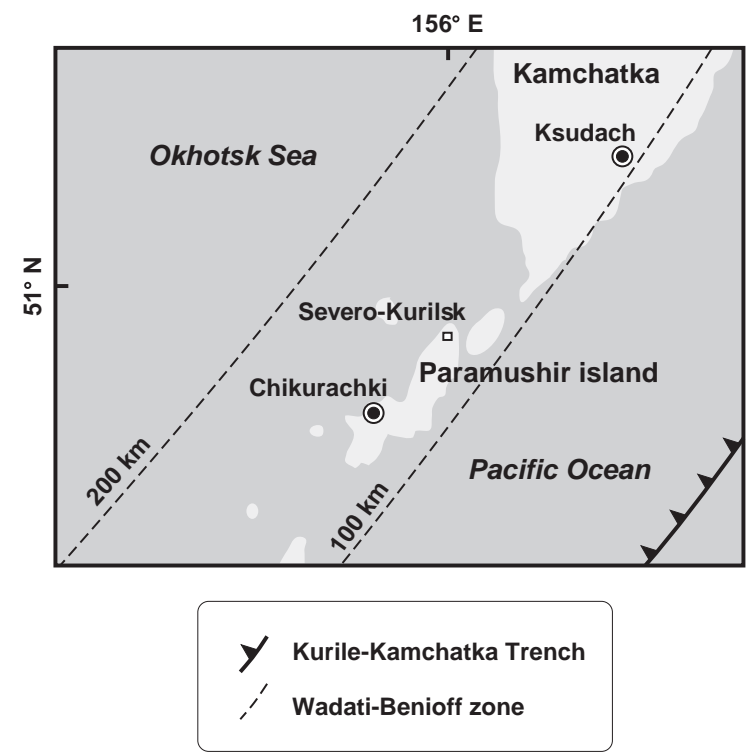

Fig. 1. Schematic map of the northern Kurile Islands with the locality of Chikurachki volcano. Contours show isobaths for the top of the Wadati-Benioff zone from Tatsumi et al. (1994). 
sequence of Pleistocene lava flows (Gorshkov, 1967). The cone has steep, smooth slopes, which are virtually uneroded. A shallow crater of approximately $450 \mathrm{~m}$ in diameter is located at the top of the volcano.

Since the beginning of historical records (1711 for this region), Chikurachki erupted in 1853-1859, 1958, 1961, 1964, 1973, 1986, 2002 and 2003. Belousov et al. (2003) distinguished two dominant eruptive styles of the volcano based on descriptions of the historical eruptions and investigation of the deposits: strombolian (1958, 1961, 1964, 1973, 2002, 2003) and plinian (1853 and 1986). The strombolian type eruptions typically lasted days to weeks. Heights of the eruption columns were commonly less than $500 \mathrm{~m}$ above the crater, and in exceptional cases the columns reached $3 \mathrm{~km}$ height. The pyroclastic deposits of the strombolian-type eruptions consist of basaltic ash and small bombs. The 1986 plinian eruption was described in detail by Ovsyannikov and Muravyev (1992). No direct observational data exist about the 1853 eruption, and its assignment as a plinian event is based on extent and nature of the tephra deposits in comparison with the 1986 eruption.

\subsection{Plinian deposits and eruption parameters}

\subsubsection{Fallout deposits of the 1853 and 1986 plinian eruptions}

The fallout deposits cover elliptical areas strongly elongated from the crater: the 1853 towards NE, and the 1986 towards SE (Fig. 8 in Belousov et al., 2003). The deposits are very similar. Each comprises a single, massive layer without notable grading. This indicates that the eruptions commenced and ceased abruptly, with no significant pulsations of discharge rate in the course of the eruptions. The deposits consist exclusively of juvenile material represented by well-sorted, coarse-grained ash and lapilli of basaltic scoria. The density of pyroclastic particles ranges from 1.1 to $1.9 \mathrm{~g} / \mathrm{cm}^{3}$ (average $1.5-1.6 \mathrm{~g} / \mathrm{cm}^{3}$ ) and vesicularity is $30-60 \%$ (Belousov et al., 2003). The high vesicularity and good sorting of the deposits suggest a purely magmatic mechanism for the eruptions. Belousov et al. (2003) estimated the erupted volumes of the 1853 and 1986 fallout deposits to be 0.09 and $0.12 \mathrm{~km}^{3}$, respectively, corresponding to 0.03 and $0.04 \mathrm{~km}^{3}$ dense rock equivalent (DRE).

\subsubsection{Fallout deposits of prehistoric plinian eruptions}

Soil sections around the volcano contain multiple layers of black to dark gray basaltic scoria $5-20 \mathrm{~cm}$ thick, locally as thick as $2.5 \mathrm{~m}$. These deposits contain well-sorted, coarse-grained ash and lapilli very similar to those of the 1853 and 1986 fallout. The deposits are interpreted by Belousov et al. (2003) as fallout deposits of prehistoric plinian eruptions of Chikurachki volcano, which is the only active basaltic volcano in the region. Based on the thickness of paleosols separating the fallout layers as well as several $\mathrm{C}^{14}$ ages obtained, Belousov et al. (2003) concluded that the intervals between plinian eruptions of Chikurachki volcano comprised 100-200 years during the last several thousands years.

\subsection{Samples studied}

\subsubsection{Whole rocks}

This study is based on analyses of glassy lapilli from the 1853 (sample CHK3/4), 1986 (sample CHK16) and two prehistoric fallout deposits (samples CHK13/1 and $13 / 2$ ). The lapilli are porphyritic. The groundmass is vitreous to devitrified, strongly vesiculated (20-30 vol.\% vesicles) and has variable contents of microphenocrysts and microlites (up to $200 \mu \mathrm{m}$ ) consisting of needle-like plagioclase, rounded olivine, prismatic to elongated augite and isometric opaque grains. Phenocrysts are mostly $0.2-3.0 \mathrm{~mm}$ in size and dominated by plagioclase (Pl, 70-90\% of all phenocrysts), which forms euhedral, elongated, twinned grains occurring as single crystals or as clusters of grains. Other phenocryst phases include rare, subhedral to rounded olivine $(\mathrm{Ol} ; 0.2-0.5 \mathrm{~mm})$, and euhedral, prismatic to broken, resorbed clinopyroxene (Cpx; 0.3-1.0 mm) (Fig. 2a). Orthopyroxene (Opx) phenocrysts occur as isolated, subhedral to rounded grains in samples CHK3/4, 13/1 and $13 / 2$ but they are very abundant in sample CHK 16 (up to $20 \%$ of all phenocrysts). Fe-Ti oxides also occur as phenocrysts (up to $0.3 \mathrm{~mm}$ ) in amounts ranging from a few to a few tens of grains per thin section.

\subsubsection{Inclusions in minerals}

We studied exclusively primary inclusions of melt and trapped crystals which were found in all major phenocryst phases (Fig. 2b-d). Due to rapid cooling of the magma on eruption, nearly all melt inclusions are naturally quenched to glass. However, some lapilli 

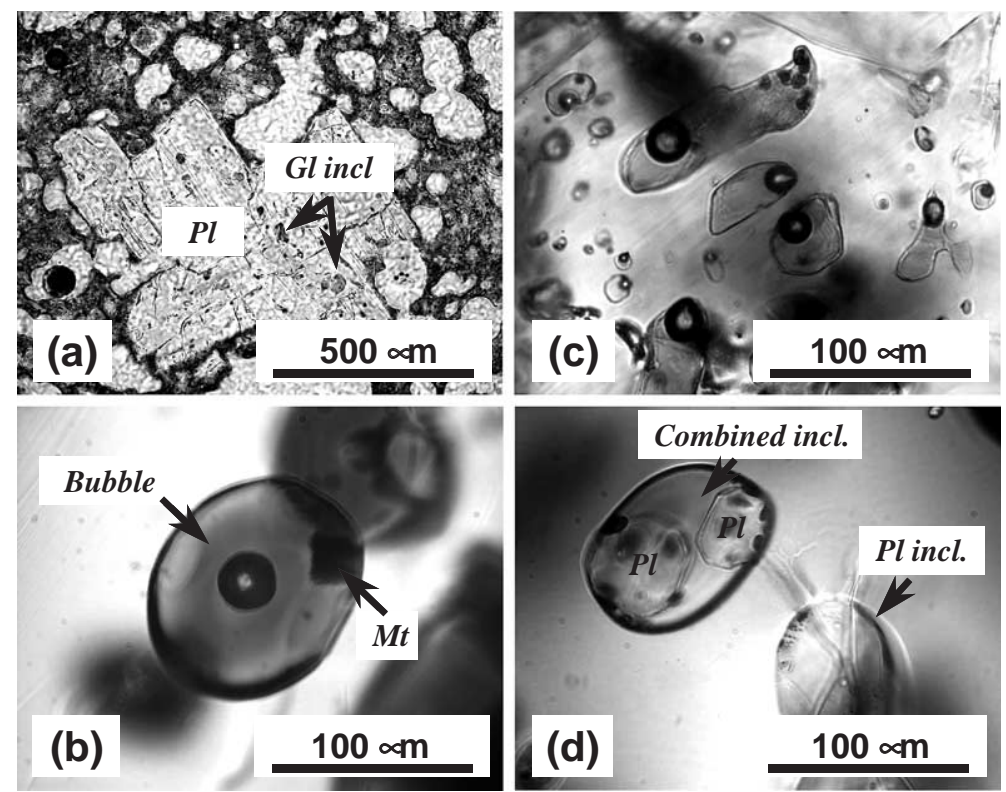

Fig. 2. Photomicrographs of typical inclusions of melt (glass) and crystals in olivine and plagioclase (Pl) phenocrysts from glassy lapilli. (a) Plagioclase phenocrysts containing glass inclusions in strongly vesiculated glassy lapilli; (b) Glass inclusion in olivine containing a gas bubble and a small crystal of magnetite (Mt); (c) Series of primary glass inclusions in plagioclase; (d) Olivine host with a glass inclusion containing two plagioclase crystals (accidentally trapped, not daughter phases) and a separate inclusion of Pl crystal.

from the same tephra samples contain multiphase inclusions composed of glass, daughter crystals of sub-micrometer size and one or more shrinkage bubbles, along with some completely recrystallized melt inclusions. This difference in inclusion appearance may reflect local differences in the cooling rate within tephra fallout. Melt inclusions in O1, Cpx and Opx hosts are commonly rounded and elliptical (Fig. 2b); those in $\mathrm{Pl}$ and $\mathrm{Cpx}$ phenocrysts tend to have more irregular, elongate shapes (Fig. 2c). The size of inclusions ranges from a few microns to about $150 \mu \mathrm{m}$. They are present as single individuals and also as groups of inclusions. For this study, we analyzed only well-quenched glassy inclusions lacking visible silicate daughter crystals, although some contained very small grains of $\mathrm{Fe}-\mathrm{Ti}$ oxides ( 1 to $10 \mu \mathrm{m}$ in size) whose formation is attributed to oxidation due to post-entrapment H-loss (discussed below).

Trapped crystalline inclusions occur either individually or in combination with a melt phase (Fig. 2d). Particularly common are inclusions of $\mathrm{Fe}-\mathrm{Ti}$ oxides, which are present in all phenocryst types, whereas the more scarce inclusions of $\mathrm{Pl}, \mathrm{Cpx}$ and $\mathrm{Opx}$ occur in $\mathrm{Ol}$ and Cpx hosts. Low-density gas inclusions (spherical or irregular shapes up to $15 \mu \mathrm{m}$ in size), and glass inclusions having anomalously large gas bubbles occur in some $\mathrm{Ol}$ and $\mathrm{Pl}$ phenocrysts. Their presence suggests oversaturation of magma with a fluid phase and partial degassing at the stage of host mineral crystallization.

\section{Analytical techniques}

\subsection{Electron probe microanalysis}

Major elements in minerals and glasses were analyzed using the Cameca SX-100 electron microprobe at the GeoForschungsZentrum Potsdam (GFZ) and the Jeol Superprobe JXA-8200 microprobe at the Max Planck Institute for Chemistry (MPI) in Mainz. Accelerating voltage was $15 \mathrm{kV}$, and the beam current and beam size were $20 \mathrm{nA}$ and $1-2 \mu \mathrm{m}$ for minerals, 10-12 nA and 10-15 $\mu \mathrm{m}$ for glass analyses at GFZ. The conditions at MPI were the same for all phases except olivine and spinel, which were analyzed at 20 $\mathrm{kV}$ and $20 \mathrm{nA}$. Counting times were $20-60 \mathrm{~s}$ depending on element concentration. Standards used were the 
Cameca and Jeol sets (mostly synthetic and natural minerals and oxides), as well as the Smithsonian set of natural glasses and minerals (Jarosewich et al., 1980). Typical analytical uncertainties were better than 1-2\% for $\mathrm{SiO}_{2}, \mathrm{Al}_{2} \mathrm{O}_{3}, \mathrm{MgO}, \mathrm{CaO}, 2-4 \%$ for $\mathrm{TiO}_{2}, \mathrm{FeO}$ and $\mathrm{Na}_{2} \mathrm{O}$, approximately $5-10 \%$ for $\mathrm{K}_{2} \mathrm{O}$ and $\mathrm{P}_{2} \mathrm{O}_{5}$, and approximately $20 \%$ for $\mathrm{MnO}$.

Sulfur and chlorine in inclusion and groundmass glasses were analyzed in the same block with major elements, using $60 \mathrm{~s}$ counting times on peaks and $30 \mathrm{~s}$ on background. Under the conditions applied, the detection limit was around 250-300 ppm for both $\mathrm{S}$ and $\mathrm{Cl}$. As reference materials to control precision and accuracy of the $\mathrm{S}$ and $\mathrm{Cl}$ measurements, we used the
USNM 111240/52 VG-2 basaltic glass (Dixon et al., 1991; Thordarson et al., 1996) and KE12 pantellerite (Mosbah et al., 1991). Our results show good agreement between the GFZ and MPI microprobes, and with published values for these glasses. For VG-2, we obtained $0.148 \pm 0.011$ wt. $\% \mathrm{~S}$ and $0.028 \pm 0.008$ wt. $\% \mathrm{Cl}(n=49)$ with the GFZ microprobe, and $0.139 \pm 0.010$ wt. $\% \mathrm{~S}$ and $0.031 \pm 0.004$ wt. $\% \mathrm{Cl}$ $(n=30)$ at MPI. Published values for VG-2 (Dixon et al., 1991; Thordarson et al., 1996) are 0.134-0.137 wt.\% $\mathrm{S}$ and $0.029-0.032$ wt.\% Cl. The pantellerite KE12 reference glass $(0.323$ wt.\% Cl; Mosbah et al., 1991 ) yielded values of $0.324 \pm 0.004$ wt. $\% \mathrm{Cl}$ $(n=13)$ with the MPI microprobe, and was not ana-
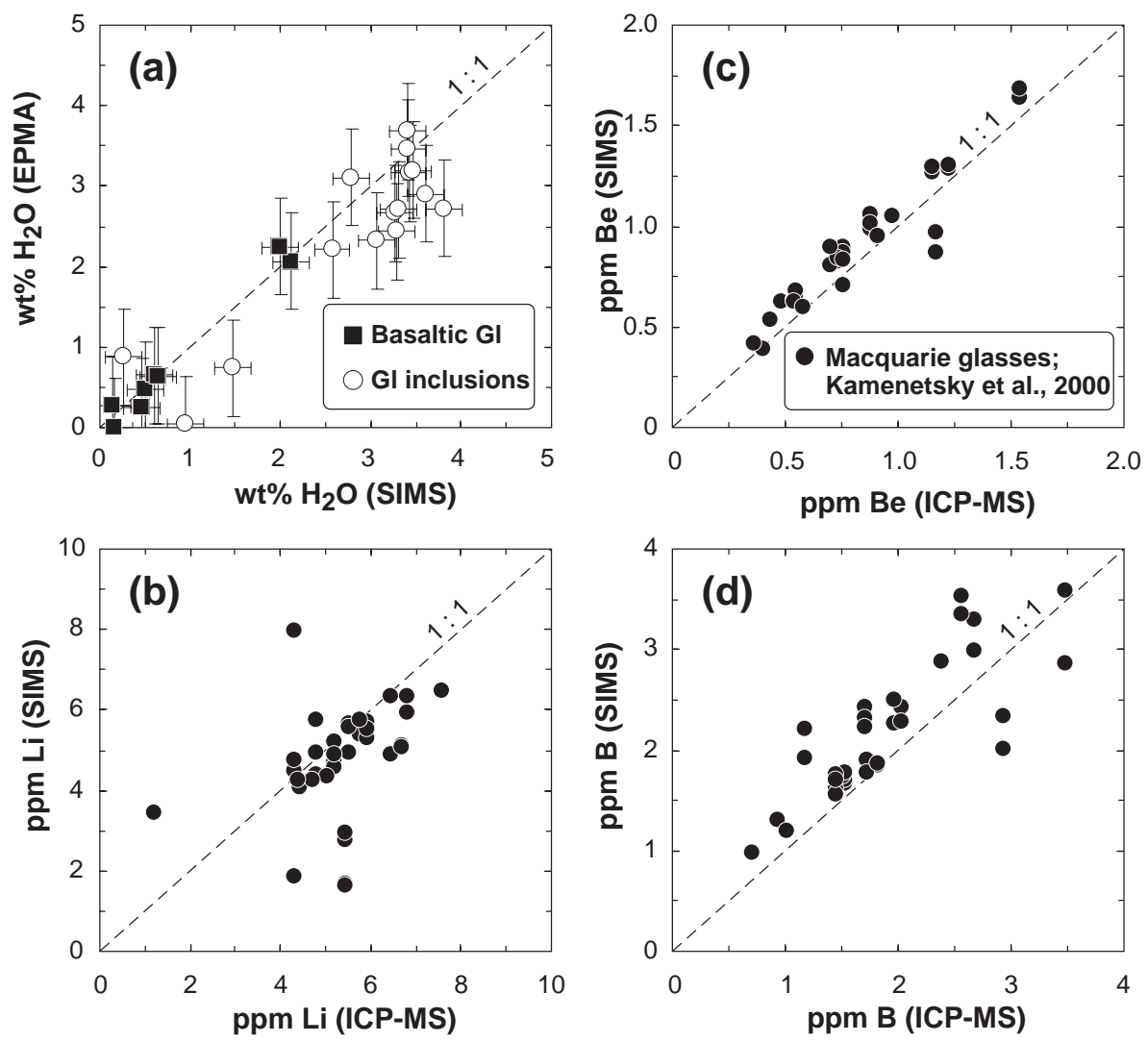

Fig. 3. (a) Comparison of $\mathrm{H}_{2} \mathrm{O}$ determinations by secondary ion mass spectrometry (SIMS) and electron microprobe (EPMA) using "by difference method". Basaltic Gl=basaltic glasses with known $\mathrm{H}_{2} \mathrm{O}$ contents (some were used for SIMS calibration; Table 2). Gl inclusions=Chikurachki glass inclusions (Table 8). Error bars represent average cumulative $2 \sigma$-error of $\pm 1.2 \mathrm{wt} . \% \mathrm{H}_{2} \mathrm{O}$ for EPMA "by difference" and \pm 0.2 wt.\% $\mathrm{H}_{2} \mathrm{O}$ for SIMS. (b-d) The comparison of whole-rock, ICP-MS and single grain, SIMS determinations of Li, Be and B concentrations in Macquarie basaltic glasses with 48-51 wt.\% $\mathrm{SiO}_{2}$ (ICP-MS data from Kamenetsky et al., 2000 and V. Kamenetsky, personal communication, 2001). The good consistency of results demonstrates no significant matrix effect (exceeding $\pm 2 \sigma$ cumulative analytical error of both methods) for the calibration curves obtained from analyses of reference glasses with 72-73 wt.\% $\mathrm{SiO}_{2}$ (Table 1). The data points deviating from the 1:1line in panel (b) are glasses exhibiting signs of secondary, low-temperature alteration. 
lyzed at GFZ. The total analytical error of $\mathrm{S}$ and $\mathrm{Cl}$, including both precision and accuracy of measurements, was better than $10 \%$ relative, or up to $20 \%$ relative for lower concentrations (i.e., $<500 \mathrm{ppm}$ ).

Concentrations of $\mathrm{H}_{2} \mathrm{O}$ were determined by SIMS on several inclusions (see below) and were also estimated by the "difference" method from EPMA analyses (e.g., Devine et al., 1995). Based on the EPMA analyses of standard glasses used for $\mathrm{H}_{2} \mathrm{O}$ calibration by SIMS and comparisons of glass inclusions analyzed by both EPMA and SIMS, we estimate that the uncertainty of the "difference" method in our case was less than 1.2 wt. $\% \mathrm{H}_{2} \mathrm{O}$ ( $2 \sigma$-error Fig. $\left.3 \mathrm{a}\right)$, about the same as reported by Devine et al. (1995) and Straub and Layne (2003).

\subsection{Secondary ion mass spectrometry}

\subsubsection{Li, Be and B concentrations}

Glass inclusions were analyzed for $\mathrm{Li}, \mathrm{Be}$ and $\mathrm{B}$ concentrations using the Cameca ims $6 \mathrm{f}$ instrument at GFZ following the technique described by Chaussidon and Libourel (1993). Standards and unknown samples were sputtered with a nominal $12.5 \mathrm{kV}^{16} \mathrm{O}^{-}$primary beam of $\sim 2-10 \mathrm{nA}$ current and $\sim 15-20 \mu \mathrm{m}$ in diameter. The energy slit was centered and opened at 25 to $50 \mathrm{~V}$.
A $150 \mu \mathrm{m}$ contrast aperture and a $750 \mu \mathrm{m}$ field aperture were used, giving an approximately $60 \mu \mathrm{m}$ field of view. Secondary, high-energy $(>80 \mathrm{eV}){ }^{7} \mathrm{Li}^{+},{ }^{9} \mathrm{Be}^{+}$, ${ }^{11} \mathrm{~B}^{+}$and ${ }^{30} \mathrm{Si}^{+}$ions were accelerated by $10 \mathrm{kV}$ and analyzed at a mass resolving power $M / \Delta M \sim 1650$ 1750 with counting times of $2,4,4$ and $2 \mathrm{~s}$, respectively. Ion intensities were corrected for the electron multiplier dead time (14 ns). Typical ion intensities observed under these conditions were 200-300 cps for ${ }^{7} \mathrm{Li}^{+}$and ${ }^{11} \mathrm{~B}^{+}, 10 \mathrm{cps}$ for ${ }^{9} \mathrm{Be}^{+}$and $3 \times 10^{4}$ cps for ${ }^{30} \mathrm{Si}^{+}$. Measurements were preceded by a $300 \mathrm{~s}$ unrastered preburn to remove gold coating and any surface contamination. Each measurement comprised 70 to 100 mass cycles. Element concentrations relative to $\mathrm{SiO}_{2}$ were calculated using calibration curves obtained by linear regressions from a set of 6 reference glasses (Table 1). The observed internal precision and external reproducibility of $\mathrm{Li} / \mathrm{Si}, \mathrm{Be} / \mathrm{Si}$ and $\mathrm{B} / \mathrm{Si}$ ratio measurements was better than $5 \%$. Variations of primary beam intensity, between 2 and $10 \mathrm{nA}$ depending on element concentrations in a given reference material, were found to have no systematic influence on the relative sensitivity factors (RSF, defined as the ratio of total ionic intensity for a given element to the ionic intensity of $\mathrm{Si}$ divided by the respective element ratios in atomic concentration for the material; i.e.,

Table 1

SIMS reference materials used for calibration of $\mathrm{Li}, \mathrm{Be}$ and $\mathrm{B}$

\begin{tabular}{|c|c|c|c|c|c|c|c|c|c|c|c|c|c|c|}
\hline \multirow{3}{*}{$\begin{array}{l}\text { Reference } \\
\text { material }^{\text {a }}\end{array}$} & \multirow[t]{3}{*}{$N^{\mathrm{b}}$} & \multirow{2}{*}{$\begin{array}{l}\mathrm{SiO}_{2} \\
\text { (wt.\%) }\end{array}$} & \multirow{2}{*}{$\begin{array}{l}\mathrm{Li} \\
\mathrm{ppm}\end{array}$} & \multirow{2}{*}{$\begin{array}{l}\mathrm{Be} \\
\mathrm{ppm}\end{array}$} & \multirow{2}{*}{$\begin{array}{l}\text { B } \\
\text { ppm }\end{array}$} & \multicolumn{2}{|l|}{${ }^{7} \mathrm{Li}^{+} /{ }^{30} \mathrm{Si}^{+}$} & \multicolumn{2}{|l|}{${ }^{9} \mathrm{Be}^{+} /{ }^{30} \mathrm{Si}^{+}$} & \multicolumn{2}{|l|}{${ }^{11} \mathrm{~B}^{+} /{ }^{30} \mathrm{Si}^{+}$} & \multirow[t]{2}{*}{$\mathrm{Li}$} & \multirow[t]{2}{*}{$\mathrm{Be}$} & \multirow[t]{2}{*}{ B } \\
\hline & & & & & & ion ratio & RSD & ion ratio & RSD & ion ratio & RSD & & & \\
\hline & & \multicolumn{4}{|c|}{ Reference values } & \multicolumn{6}{|c|}{ Measured values $^{\mathrm{c}}$} & \multicolumn{3}{|c|}{ Relative sensitivity factors ${ }^{\mathrm{d}}$} \\
\hline JV1 & 2 & 72.26 & 1719 & 41.1 & 1920 & $9.20 \mathrm{E}-01$ & 0.3 & $9.82 \mathrm{E}-03$ & 0.3 & $1.28 \mathrm{E}-01$ & 0.0 & 1.491 & 0.800 & 0.334 \\
\hline DK89 & 4 & 73.04 & 171 & 7.8 & 14 & $4.28 \mathrm{E}-02$ & 1.4 & $1.94 \mathrm{E}-03$ & 1.3 & $1.09 \mathrm{E}-03$ & 3.2 & 0.705 & 0.842 & 0.395 \\
\hline GB4 & 4 & 72.94 & 384 & 11.3 & 970 & $9.64 \mathrm{E}-02$ & 4.6 & $2.75 \mathrm{E}-03$ & 2.2 & $6.83 \mathrm{E}-02$ & 7.1 & 0.706 & 0.823 & 0.357 \\
\hline NBS 610 & 2 & 72.00 & 487.9 & 452 & 351 & $1.28 \mathrm{E}-01$ & 2.0 & $1.06 \mathrm{E}-01$ & 0.6 & $2.84 \mathrm{E}-02$ & 0.1 & 0.728 & 0.783 & 0.404 \\
\hline NBS 612 & 2 & 72.00 & 41.8 & 38.3 & 32 & $1.02 \mathrm{E}-02$ & 0.1 & $8.58 \mathrm{E}-03$ & 0.3 & $2.77 \mathrm{E}-03$ & 0.3 & 0.677 & 0.748 & 0.433 \\
\hline
\end{tabular}

${ }^{a}$ Natural and synthetic glasses used for calibration: JV1 is an obsidian glass (Pichavant, 1987); GB4 and DK89 are CRPG internal reference glasses (Chaussidon and Libourel, 1993; Chaussidon and Jambon, 1994; M. Chaussidon, pers. communication, 1998), SRM 610 and SRM 612 are NIST certified glass reference materials (Gladney and Roelandts, 1987; Pearce et al., 1997). The nominal $\mathrm{SiO}_{2}$ content of 72 wt.\% in SRM 610 and 612 glasses is that of the matrix doped by trace elements $(50-500 \mathrm{ppm})$.

${ }^{\mathrm{b}}$ In this table and Table 2, $N=$ number of replicates over the entire analytical session.

${ }^{c}$ Ion ratio represents uncorrected ion isotopic ratios measured with ion probe, RSD (given in percent)=relative standard deviation obtained during replicate analyses of reference materials; it represents 1 sigma standard deviation $[1 \sigma \mathrm{SD}]$ calculated for three measurements and indicates a total range in case of two measurements divided over the average ion ratio of a given element.

${ }^{d}$ In this table and Table 2, relative sensitivity factors are defined as the ratios of element total ionic intensity normalized to ionic intensity of Si over element atomic concentration normalized to $\mathrm{Si}$ atomic concentration [i.e., $\left(\mathrm{Li}^{+} / \mathrm{Si}^{+}\right) /(\mathrm{Li} / \mathrm{Si})$, etc.]. The value given in italic was not considered during calculation of analytical precision. The following calibration curves were obtained: $\log (\mathrm{ppm} \mathrm{Li} / \mathrm{wt} . \%$ $\left.\mathrm{SiO}_{2}\right)=0.86216 \times \log \left({ }^{7} \mathrm{Li}^{+} /{ }^{30} \mathrm{Si}^{+}\right)+1.53457, R=0.996 ; \log \left(\mathrm{ppm} \mathrm{Be} / \mathrm{wt} . \% \mathrm{SiO}_{2}\right)=0.97842 \times \log \left({ }^{9} \mathrm{Be}^{+} /{ }^{30} \mathrm{Si}^{+}\right)+1.71894, R=0.999 ; \log (\mathrm{ppm}$ $\left.\mathrm{B} / \mathrm{wt} . \% \mathrm{SiO}_{2}\right)=0.94018 \times \log \left({ }^{11} \mathrm{~B}^{+} /{ }^{30} \mathrm{Si}^{+}\right)+2.17711, R=0.999$. 
$\left(\mathrm{Li}^{+} / \mathrm{Si}^{+}\right) /(\mathrm{Li} / \mathrm{Si})$. Possible chemical matrix effects due to the difference between relatively low-silica (50.4-58.5 wt.\% $\mathrm{SiO}_{2}$ ) glass inclusions and the highsilica reference glasses used for calibration (72-73 wt.\% $\mathrm{SiO}_{2}$ ) were assessed and are not believed to exceed the analytical uncertainty of $\pm 10 \%$ relative $(2 \sigma)$. This follows from SIMS analyses of secondary

Table 2

$\mathrm{H}_{2} \mathrm{O}$ calibration by SIMS

\begin{tabular}{|c|c|c|c|c|c|c|}
\hline \multirow{3}{*}{$\begin{array}{l}\text { Reference } \\
\text { material }^{\mathrm{a}}\end{array}$} & \multirow[t]{3}{*}{$N$} & \multirow{2}{*}{$\begin{array}{l}\mathrm{SiO}_{2} \\
\text { wt. } \%\end{array}$} & \multirow{2}{*}{$\begin{array}{l}\mathrm{H}_{2} \mathrm{O} \\
\text { wt. } \%\end{array}$} & \multicolumn{2}{|l|}{${ }^{1} \mathrm{H}^{+} /{ }^{30} \mathrm{Si}^{+\mathrm{b}}$} & \multirow[t]{3}{*}{$\mathrm{H} \mathrm{RSF}^{\mathrm{d}}$} \\
\hline & & & & ion ratio & $\operatorname{RSD}^{c}$ & \\
\hline & & \multicolumn{2}{|c|}{$\begin{array}{l}\text { Reference } \\
\text { values }\end{array}$} & \multicolumn{2}{|l|}{$\begin{array}{l}\text { Measured } \\
\text { values }\end{array}$} & \\
\hline \multicolumn{7}{|c|}{24 October, 2002} \\
\hline Olivine & 1 & 38.06 & - & $1.470 \mathrm{E}-02$ & 3.4 & - \\
\hline R316 & 3 & 52.88 & 2.11 & $7.689 \mathrm{E}-01$ & 4.8 & $9.991 \mathrm{E}-03$ \\
\hline TRDS-27 & 4 & 53.87 & 2.10 & $8.219 \mathrm{E}-01$ & 4.8 & $1.093 \mathrm{E}-02$ \\
\hline $29-3$ & 2 & 52.39 & 0.50 & $1.831 \mathrm{E}-01$ & 3.9 & $9.947 \mathrm{E}-03$ \\
\hline $20-1$ & 2 & 49.71 & 0.11 & $5.027 \mathrm{E}-02$ & 2.5 & $1.178 \mathrm{E}-02$ \\
\hline
\end{tabular}

26 October, 2002

$\begin{array}{lllllll}\text { Olivine } & 1 & 38.61 & - & 1.413 \mathrm{E}-02 & 3.3 & - \\ \text { TRDS-27 } & 2 & 53.87 & 2.10 & 8.188 \mathrm{E}-01 & 1.3 & 1.089 \mathrm{E}-02 \\ \text { TRDS-15 } & 2 & 54.72 & 2.10 & 7.787 \mathrm{E}-01 & 1.6 & 1.052 \mathrm{E}-02 \\ 30-2 & 2 & 51.71 & 0.60 & 2.046 \mathrm{E}-01 & 3.1 & 9.143 \mathrm{E}-03 \\ 40-2 & 3 & 50.38 & 0.15 & 3.978 \mathrm{E}-02 & 6.7 & 6.927 \mathrm{E}-03\end{array}$

9 December, 2002

$\begin{array}{lllllrl}\text { Olivine } & 1 & 40.16 & - & 5.211 \mathrm{E}-03 & 12.7 & - \\ 30-2 & 2 & 51.71 & 0.60 & 1.976 \mathrm{E}-01 & 1.7 & 8.830 \mathrm{E}-03 \\ 40-2 & 2 & 50.38 & 0.15 & 4.404 \mathrm{E}-02 & 2.6 & 7.669 \mathrm{E}-03 \\ \text { TRDS-27 } & 2 & 53.87 & 2.10 & 7.508 \mathrm{E}-01 & 1.3 & 9.986 \mathrm{E}-03 \\ \text { TRDS-15 } & 2 & 54.72 & 2.10 & 7.654 \mathrm{E}-01 & 2.4 & 1.034 \mathrm{E}-02\end{array}$

10 December, 2002

\begin{tabular}{lllllll} 
Ab10 & 3 & 59.54 & 5.50 & $1.478 \mathrm{E}-00$ & 2.9 & $8.296 \mathrm{E}-03$ \\
Ab25 & 3 & 63.96 & 6.72 & $1.693 \mathrm{E}-00$ & 2.9 & $8.355 \mathrm{E}-03$ \\
Ab100 & 3 & 66.28 & 8.48 & $2.260 \mathrm{E}-00$ & 1.8 & $9.159 \mathrm{E}-03$ \\
\multicolumn{7}{l}{ 12 December, 2002} \\
Olivine & 1 & 40.00 & - & $1.229 \mathrm{E}-02$ & 3.8 & - \\
AOQ & 3 & 75.07 & 1.86 & $5.257 \mathrm{E}-01$ & 2.6 & $1.100 \mathrm{E}-02$ \\
P1 & 3 & 68.72 & 2.09 & $5.974 \mathrm{E}-01$ & 1.2 & $1.018 \mathrm{E}-02$ \\
P2 & 3 & 68.10 & 4.00 & $9.912 \mathrm{E}-01$ & 1.4 & $8.750 \mathrm{E}-03$ \\
HPG6 & 3 & 73.94 & 6.57 & $1.699 \mathrm{E}-00$ & 4.3 & $9.914 \mathrm{E}-03$ \\
HPG12 & 3 & 73.89 & 6.13 & $1.520 \mathrm{E}-00$ & 1.8 & $9.500 \mathrm{E}-03$
\end{tabular}

14 December, 2002

\begin{tabular}{lllllll} 
Olivine & 1 & 40.16 & - & $7.582 \mathrm{E}-03$ & 5.2 & - \\
$30-2$ & 2 & 51.71 & 0.60 & $1.713 \mathrm{E}-01$ & 0.9 & $7.655 \mathrm{E}-03$ \\
$20-1$ & 1 & 49.71 & 0.11 & $2.153 \mathrm{E}-02$ & 1.8 & $5.045 \mathrm{E}-03$ \\
$40-2$ & 1 & 50.38 & 0.15 & $4.063 \mathrm{E}-02$ & 4.3 & $7.075 \mathrm{E}-03$ \\
\hline
\end{tabular}

reference glasses and Macquarie tholeiitic glasses with 48-51 wt.\% $\mathrm{SiO}_{2}, 4.3-7.6$ ppm Li, 0.4-1.5 ppm Be, and $0.7-3.5$ ppm B (ICP-MS data, Kamenetsky et al., 2000; V. Kamenetsky, personal communication, 2001), for which the same set of high-Si glass standards was employed (Fig. 3b-d). Chaussidon and Libourel (1993), Chaussidon and Jambon (1994) and Straub and Layne (2003) also concluded that chemical matrix effect on SIMS analyses of Be and B for basaltic to rhyolitic glasses was less than the external precision.

\subsection{2. $\mathrm{H}_{2} \mathrm{O}$ and trace elements}

The concentrations of $\mathrm{H}_{2} \mathrm{O}$ and trace elements (REE, Nb, Th, Sr, Y, Zr, V and Cr) were determined using the Cameca ims $3 f$ instrument at the MPI in Mainz. For $\mathrm{H}_{2} \mathrm{O}$ analysis, conditions were similar to those described by Sobolev and Chaussidon (1996), with $12.5 \mathrm{kV}$ accelerating voltage for the ${ }^{16} \mathrm{O}^{-}$primary beam, $4.5 \mathrm{kV}$ secondary accelerating voltage, -80 $\mathrm{V}$ offset and $M / \Delta M \approx 300$. The energy slit was centered and opened to $25 \mathrm{~V}$. A $150 \mu \mathrm{m}$ contrast aperture and a $750 \mu \mathrm{m}$ field aperture were used. Analyses were performed in three blocks of 6 cycles over the masses ${ }^{1} \mathrm{H},{ }^{30} \mathrm{Si}$ and ${ }^{47} \mathrm{Ti}$, each counted for $2 \mathrm{~s}$. Titanium was monitored to detect and, if necessary, correct for

Notes to Table 2:

${ }^{a}$ Natural glasses used for calibration: R316, TRDS-27 and TRDS-15 are pillow rim glasses from Troodos ophiolite, Cyprus (Rautenschlein et al., 1985; Sobolev et al., 1993); 20-1, 29-3, 30-2 and 40-2 are MORB glasses (Danyushevsky et al., 1993; A.V. Sobolev, personal communication, 1994); Ab10, Ab25, $\mathrm{Ab} 100=$ synthetic quartz-albite glasses, $\mathrm{AOQ}=$ synthetic quartzalbite-orthoclase glass; P1, P2=synthetic peraluminous glasses, HPG6, HPG12=synthetic haplogranitic glasses provided by R. Thomas (personal communication, 2002) and F. Holtz (personal communication, 2003); for background information on experimental and analytical conditions see Holtz et al. (1992, 2001), and references therein). The $\mathrm{SiO}_{2}$ contents in the individual glass chips used for calibration were re-analyzed by electron microprobe.

${ }^{\mathrm{b}}{ }^{1} \mathrm{H}^{+} /{ }^{30} \mathrm{Si}^{+}=$ion ratio measured and then corrected for $\mathrm{H}_{2} \mathrm{O}$ background on olivine.

${ }^{c} \mathrm{RSD}=$ relative standard deviation obtained during replicate analyses of reference materials used for calibration; it represents $1 \sigma \mathrm{SD}$ calculated for three measurements, indicates a total range in case of two measurements and represents internal analytical precision in case of one measurement, normalized to the average ${ }^{1} \mathrm{H}^{+} /{ }^{30} \mathrm{Si}^{+}$ion ratio.

${ }^{\mathrm{d}} \mathrm{RSF}=$ relative sensitivity factors of $\mathrm{H}$ normalized to $\mathrm{Si}$ are expressed as in Table 1. The following calibration curve was obtained: wt. $\% \mathrm{H}_{2} \mathrm{O} /$ wt. $\% \mathrm{SiO}_{2}=0.05698 \times{ }^{1} \mathrm{H}^{+} /{ }^{30} \mathrm{Si}^{+}, R=0.994$. 
overlap with host minerals in the case of small $(<40$ $\mu \mathrm{m})$ inclusions. A set of natural and synthetic glasses with water concentrations ranging from 0.1 to 8.5 wt. $\% \mathrm{H}_{2} \mathrm{O}$ (Table 2) was used for calibration. The olivine host grains were repeatedly analyzed throughout each session to monitor the $\mathrm{H}_{2} \mathrm{O}$ background level, and inclusion analyses were started when $\mathrm{H}_{2} \mathrm{O}$ concentration measured on olivine was equivalent to, or lower than, 0.03 wt.\%. Typical count rates were $3-5 \times 10^{4}$ cps on the ${ }^{1} \mathrm{H}$ peak, $2-4 \times 10^{2}$ on the ${ }^{47} \mathrm{Ti}$ peak and $2-3 \times 10^{4}$ on the ${ }^{30} \mathrm{Si}$ peak. The external precision, assessed from multiple measurements of reference glasses, was better then $7 \%$ relative (Table 2 ). We found no significant chemical matrix effect for $\mathrm{H}_{2} \mathrm{O}$ due to the difference in $\mathrm{SiO}_{2}$ content between unknown and reference glasses, based on the excellent linear correlation $(R=0.994)$ between $\mathrm{H}_{2} \mathrm{O} / \mathrm{SiO}_{2}$ and ${ }^{1} \mathrm{H}^{+} /{ }^{30} \mathrm{Si}^{+}$ratios and similarity of slopes of the calibration line between out study (0.05698; Table 2) and those obtained in the CRNS-Nancy SIMS laboratory using different reference glasses $(0.03327$ by Sobolev and Chaussidon, 1996; 0.04447 by Gurenko et al., 1998; 0.0649 by Gurenko and Schmincke, 2002).

The analysis of trace elements employed similar instrument settings as for $\mathrm{H}_{2} \mathrm{O}$, except that a larger field aperture $(1800 \mu \mathrm{m})$ was used. Each analysis consisted of 5 sequential scans of the masses ${ }^{16} \mathrm{O}$, ${ }^{30} \mathrm{Si},{ }^{35} \mathrm{Cl},{ }^{39} \mathrm{~K},{ }^{44} \mathrm{Ca},{ }^{47} \mathrm{Ti},{ }^{51} \mathrm{~V},{ }^{52} \mathrm{Cr},{ }^{88} \mathrm{Sr},{ }^{89} \mathrm{Y},{ }^{90} \mathrm{Zr}$, ${ }^{93} \mathrm{Nb}$, then the REE masses from 133 to 180 and finally mass ${ }^{232} \mathrm{Th}$. The remaining oxide interferences, e.g., light rare earth element (LREE) oxides interfering with heavy rare earth elements (HREE) were

Table 3

Precision and accuracy of trace element analyses by SIMS

\begin{tabular}{|c|c|c|c|c|c|c|c|c|c|c|c|c|c|}
\hline \multirow{2}{*}{$\begin{array}{l}\text { Elements } \\
(\mathrm{ppm})\end{array}$} & \multirow{2}{*}{$\begin{array}{l}\text { KL2G } \\
\text { REF }\end{array}$} & \multicolumn{4}{|c|}{$21-24$ October $2002, N=5$} & \multicolumn{4}{|c|}{ 6-8 December 2002, $N=5$} & \multicolumn{4}{|c|}{ 16-17 December 2002, $N=3$} \\
\hline & & Mean & $\pm 1 \sigma \mathrm{SD}$ & RSD & $\Delta \mathrm{RV}$ & Mean & $\pm 1 \sigma \mathrm{SD}$ & RSD & $\Delta \mathrm{RV}$ & Mean & $\pm 1 \sigma \mathrm{SD}$ & RSD & $\Delta \mathrm{RV}$ \\
\hline K & 4000 & 3816 & 91 & 2.4 & -4.6 & 3624 & 70 & 1.9 & -9.4 & 3830 & 200 & 5.2 & -4.3 \\
\hline $\mathrm{Ti}$ & 15,600 & 15,540 & 261 & 1.7 & -0.4 & 15,400 & 100 & 0.6 & -1.3 & 16,067 & 321 & 2.0 & 3.0 \\
\hline V & 370 & 262 & 7 & 2.7 & -29.2 & 249 & 4 & 1.6 & -32.7 & 263 & 7 & 2.7 & -28.9 \\
\hline $\mathrm{Cr}$ & 310 & 298 & 6 & 2.0 & -3.9 & 290 & 5 & 1.7 & -6.5 & 296 & 3 & 1.0 & -4.5 \\
\hline $\mathrm{Sr}$ & 364 & 398 & 4 & 1.0 & 9.3 & 379 & 2 & 0.5 & 4.1 & 377 & 6 & 1.6 & 3.6 \\
\hline $\mathrm{Ba}$ & 123 & 137 & 5 & 3.6 & 11.4 & 124 & 2 & 1.6 & 0.8 & 122 & 3 & 2.5 & -0.8 \\
\hline Y & 26.8 & 24.9 & 0.5 & 2.0 & -7.1 & 24.1 & 0.2 & 0.8 & -10.1 & 24.4 & 0.6 & 2.5 & -9.0 \\
\hline $\mathrm{Zr}$ & 159 & 156 & 5 & 3.2 & -1.9 & 150 & 2 & 1.3 & -5.7 & 156 & 4 & 2.6 & -1.9 \\
\hline $\mathrm{Nb}$ & 15.8 & 17.7 & 0.4 & 2.3 & 12.0 & 16.2 & 0.2 & 1.2 & 2.5 & 17.2 & 0.6 & 3.5 & 8.9 \\
\hline Hf & 4.14 & 4.03 & 0.61 & 15.1 & -2.7 & 3.78 & 1.15 & 30.4 & -8.7 & 4.03 & 0.66 & 16.4 & -2.7 \\
\hline Th & 1.03 & 1.08 & 0.12 & 11.1 & 4.9 & 0.8 & 0.12 & 15.0 & -22.3 & 0.91 & 0.21 & 23.1 & -11.7 \\
\hline $\mathrm{La}$ & 13.2 & 13.5 & 0.4 & 3.0 & 2.3 & 12.8 & 0.2 & 1.6 & -3.0 & 12.7 & 0.2 & 1.6 & -3.8 \\
\hline $\mathrm{Ce}$ & 32.9 & 34.7 & 1.2 & 3.5 & 5.5 & 32.2 & 0.7 & 2.2 & -2.1 & 32.3 & 0.3 & 0.9 & -1.8 \\
\hline $\operatorname{Pr}$ & 4.71 & 5.18 & 0.22 & 4.2 & 10.0 & 4.85 & 0.14 & 2.9 & 3.0 & 4.79 & 0.11 & 2.3 & 1.7 \\
\hline $\mathrm{Nd}$ & 21.7 & 23.0 & 1.0 & 4.3 & 6.0 & 21.7 & 0.5 & 2.3 & 0.0 & 21.5 & 0.4 & 1.9 & -0.9 \\
\hline $\mathrm{Sm}$ & 5.55 & 6.05 & 0.33 & 5.5 & 9.0 & 5.71 & 0.27 & 4.7 & 2.9 & 5.44 & 0.22 & 4.0 & -2.0 \\
\hline $\mathrm{Eu}$ & 1.95 & 2.17 & 0.09 & 4.1 & 11.3 & 2.06 & 0.09 & 4.4 & 5.6 & 1.91 & 0.07 & 3.7 & -2.1 \\
\hline Gd & 6.10 & 5.93 & 0.74 & 12.5 & -2.8 & 5.58 & 0.52 & 9.3 & -8.5 & 5.3 & 0.2 & 3.8 & -13.1 \\
\hline $\mathrm{Tb}$ & 0.93 & 0.92 & 0.09 & 9.8 & -1.1 & 0.81 & 0.05 & 6.2 & -12.9 & 0.77 & 0.07 & 9.1 & -17.2 \\
\hline Dy & 5.35 & 5.64 & 0.22 & 3.9 & 5.4 & 4.98 & 0.22 & 4.4 & -6.9 & 4.87 & 0.12 & 2.5 & -9.0 \\
\hline Но & 0.99 & 1.03 & 0.04 & 3.9 & 4.0 & 0.95 & 0.06 & 6.3 & -4.0 & 0.89 & 0.06 & 6.7 & -10.1 \\
\hline Er & 2.64 & 2.77 & 0.09 & 3.2 & 4.9 & 2.34 & 0.14 & 6.0 & -11.4 & 2.44 & 0.17 & 7.0 & -7.6 \\
\hline $\mathrm{Tm}$ & 0.34 & 0.38 & 0.02 & 5.3 & 11.8 & 0.31 & 0.08 & 25.8 & -8.8 & 0.31 & 0.04 & 12.9 & -8.8 \\
\hline $\mathrm{Yb}$ & 2.13 & 2.21 & 0.12 & 5.4 & 3.8 & 1.88 & 0.13 & 6.9 & -11.7 & 1.89 & 0.15 & 7.9 & -11.3 \\
\hline $\mathrm{Lu}$ & 0.30 & 0.34 & 0.05 & 14.7 & 13.3 & 0.23 & 0.03 & 13.0 & -23.3 & 0.31 & 0.03 & 9.7 & 3.3 \\
\hline
\end{tabular}

Precision and accuracy of trace element analyses was controlled by multiple analyses of KL2-G reference glass during each analytical session; $N=$ number of replicates; REF=reference values of trace element concentrations (given in parts per million) of KL2-G from Jochum et al. (2000); Mean=average value obtained from replicate analyses of KL2-G; $1 \sigma \mathrm{SD}=1$ sigma standard deviation; RSD (given in percent)=relative standard deviation obtained during a given analytical session and defined as $100 \times 1 \sigma \mathrm{SD} /$ Mean; $\Delta \mathrm{RV}$ (given in percent)=deviation from the reference values (over a given analytical session) that characterizes the accuracy of trace element analyses and defined as $100 \times($ Mean $/$ Ref -1$)$; all analyses of melt inclusions and groundmass glasses (Table 8) were corrected for $\Delta \mathrm{RV}$ values. 
corrected by peak deconvolution (Zinner and Crozaz, 1986; Fahey et al., 1987). Relative sensitivity factors (Table 2) were determined from analyses of basaltic reference glasses (Jochum et al., 2000). Instrument drift was controlled and correction applied using daily replicate analyses of KL2-G reference glass (Table 3). The obtained analytical error was better than $10 \%$ relative for all elements except $\mathrm{Gd}$, Tm, $\mathrm{Lu}$, Hf and Th whose uncertainties range between $11 \%$ and $30 \%$ relative (Table 3 ).

\section{Mineral chemistry}

Compositions of Ol, Cpx, Opx, $\mathrm{Pl}$ and $\mathrm{Fe}-\mathrm{Ti}$ oxides are summarized in Tables $4-6$. Olivine phenocrysts range in composition from $\mathrm{Fo}_{69}$ to $\mathrm{Fo}_{78}$ with the majority (97 of 102 analyses) between $\mathrm{Fo}_{72}$ and $\mathrm{Fo}_{76}$. Olivine in the groundmass has similar forsterite contents as the phenocrysts $\left(\mathrm{Fo}_{69-73}\right.$; Table 4$)$. No systematic intra-sample difference in forsterite contents was found. Calcium contents are $0.11-0.25 \mathrm{wt} . \% \mathrm{CaO}$ in $\mathrm{Ol}$ phenocrysts and $0.18-0.74$ wt. $\% \mathrm{CaO}$ in groundmass grains; nickel concentrations are similar in both $\mathrm{Ol}$ types, ranging from the detection limit (0.01-0.02 wt. $\% \mathrm{NiO}$ ) to $0.09 \mathrm{wt} . \% \mathrm{NiO}$ (Table 4 ). These are fairly low values for $\mathrm{Ni}$ in basaltic olivines, and probably reflect rapid Ni depletion from the primary melt due to abundant crystallization of $\mathrm{Ol}$ and/or Ni-bearing sulfide early in magma evolution.

Orthopyroxenes are present only as phenocrysts in tephra from the 1853 and 1986 eruptions and they correspond chemically to bronzite with $m g$-numbers $\left[\mathrm{mg} \#=100 \times \mathrm{Mg} /\left(\mathrm{Mg}+\mathrm{Fe}_{\text {tot }}\right)\right]$ ranging from 72 to 75 (Table 4). The concentrations of $\mathrm{Ti}, \mathrm{Al}$ and $\mathrm{Ca}$ in Opx are low and slightly variable $\left(0.17-0.26\right.$ wt. $\% \mathrm{TiO}_{2}$, 1.1-1.8 wt.\% $\mathrm{Al}_{2} \mathrm{O}_{3}$ and $1.5-2.0$ wt. $\% \mathrm{CaO}$ ), but show no relation to $m g$-number. Low-Ca pyroxene of the groundmass is pigeonitic (mg\#=65-72, 0.231.0 wt. $\% \mathrm{TiO}_{2}, 1.7-5.3$ wt. $\% \mathrm{Al}_{2} \mathrm{O}_{3}$ and 3.0-9.2 wt.\% $\mathrm{CaO})$. Clinopyroxenes are augite and present as phenocrysts and as groundmass grains; their $m g$-numbers range from 63 to 77 in both cases and minor components include $0.30-1.4$ wt. $\% \mathrm{TiO}_{2}, 2.0-6.5$ wt.\% $\mathrm{Al}_{2} \mathrm{O}_{3}$ and $0.19-0.32$ wt. $\% \mathrm{Na}_{2} \mathrm{O}$ (Table 4).

Plagioclase phenocrysts range from $\mathrm{An}_{74}$ to $\mathrm{An}_{95}$, while groundmass $\mathrm{Pl}$ is considerably less calcic, with $\mathrm{An}_{50-60}$ (Table 4). Plagioclase inclusions in olivine
Table 4

Summary of silicate mineral compositions

\begin{tabular}{|c|c|c|c|c|}
\hline Sample & Chk3/4 & Chk16 & Chk13/1 & Chk13/2 \\
\hline \multicolumn{5}{|l|}{ Olivine } \\
\hline$N_{\text {phen }}$ & 39 & 4 & 35 & 24 \\
\hline Fo & $72.4-75.5$ & $71.1-74.6$ & $68.9-74.4$ & $73.1-78.3$ \\
\hline $\mathrm{CaO}$ & $0.14-0.25$ & $0.15-0.16$ & $0.13-0.23$ & $0.11-0.18$ \\
\hline $\mathrm{NiO}$ & $<0.09$ & $<0.06$ & $<0.05$ & $<0.09$ \\
\hline$N_{\mathrm{grm}}$ & 3 & 1 & - & 7 \\
\hline Fo & $68.7-71.7$ & 70.5 & - & $68.5-72.9$ \\
\hline $\mathrm{CaO}$ & $0.35-0.64$ & 0.25 & - & $0.18-0.74$ \\
\hline $\mathrm{NiO}$ & $<0.03$ & $<0.03$ & - & $<0.05$ \\
\hline \multicolumn{5}{|l|}{ Augite } \\
\hline$N_{\text {phen }}$ & 8 & 26 & - & 5 \\
\hline $\mathrm{mg} \#_{\mathrm{cpx}}$ & $72.0-76.9$ & $73.7-76.7$ & - & $74.3-74.6$ \\
\hline $\mathrm{TiO}_{2}$ & $0.30-0.54$ & $0.36-0.53$ & - & $0.54-0.56$ \\
\hline $\mathrm{Al}_{2} \mathrm{O}_{3}$ & $2.2-4.2$ & $2.0-2.9$ & - & $3.0-3.2$ \\
\hline $\mathrm{CaO}$ & $18.7-21.3$ & 18.9-20.3 & - & $19.3-19.9$ \\
\hline $\mathrm{Na}_{2} \mathrm{O}$ & $0.22-0.31$ & $0.19-0.28$ & - & $0.29-0.30$ \\
\hline$N_{\mathrm{grm}}$ & 1 & 4 & - & - \\
\hline $\mathrm{mg} \#_{\mathrm{cpx}}$ & 66.6 & $63.4-70.0$ & - & - \\
\hline $\mathrm{TiO}_{2}$ & 1.43 & $0.69-1.35$ & - & - \\
\hline $\mathrm{Al}_{2} \mathrm{O}_{3}$ & 6.5 & $4.1-6.0$ & - & - \\
\hline $\mathrm{CaO}$ & 18.0 & $10.4-13.4$ & - & - \\
\hline $\mathrm{Na}_{2} \mathrm{O}$ & $0.22-0.31$ & $0.20-0.32$ & - & - \\
\hline \multicolumn{5}{|l|}{ Pigonite } \\
\hline$N_{\mathrm{grm}}$ & 1 & 6 & - & 2 \\
\hline $\mathrm{mg} \#_{\mathrm{cpx}}$ & 65.1 & $65.2-70.3$ & - & $71.3-71.8$ \\
\hline $\mathrm{TiO}_{2}$ & 1.00 & $0.23-0.80$ & - & $0.26-0.29$ \\
\hline $\mathrm{Al}_{2} \mathrm{O}_{3}$ & 4.5 & $1.7-5.3$ & - & $1.4-1.5$ \\
\hline $\mathrm{CaO}$ & 9.2 & $4.0-9.8$ & - & $3.0-6.0$ \\
\hline $\mathrm{Na}_{2} \mathrm{O}$ & 0.29 & $0.05-0.41$ & - & $0.03-0.08$ \\
\hline
\end{tabular}

Orthopyroxene

\begin{tabular}{|c|c|c|c|c|}
\hline \\
\hline & - & 12 & - & - \\
\hline $\mathrm{mg} \#_{\text {opx }}$ & - & $71.7-75.3$ & - & - \\
\hline $\mathrm{TiO}_{2}$ & - & $0.17-0.26$ & - & - \\
\hline $\mathrm{Al}_{2} \mathrm{O}_{3}$ & - & $1.1-1.8$ & - & - \\
\hline $\mathrm{CaO}$ & - & $1.5-2.0$ & - & - \\
\hline \multicolumn{5}{|c|}{ Plagioclase } \\
\hline$N_{\text {phen }}$ & 18 & 79 & 34 & 21 \\
\hline An & $81.8-94.9$ & $80.4-93.0$ & $73.9-91.6$ & 82.9-91.2 \\
\hline$N_{\mathrm{grm}}$ & 5 & 4 & - & 2 \\
\hline An & $51.5-59.0$ & $49.8-60.8$ & - & $54.6-56.3$ \\
\hline
\end{tabular}

Individual analyses are available on request; $N=$ number of microprobe analyses, phen=phenocrysts, grm $=$ groundmass; $\mathrm{Fo}=\mathrm{mol} \%$ forsterite in olivine, $\mathrm{mg} \#=100 \times \mathrm{Mg} /\left(\mathrm{Mg}+\mathrm{Fe}_{\mathrm{tot}}\right)$, atomic ratio, where $\mathrm{Fe}_{\text {tot }}$ is a total iron as $\mathrm{FeO} ; \mathrm{An}=\mathrm{mol} \%$ anorthite, $\mathrm{TiO}_{2}$, $\mathrm{Al}_{2} \mathrm{O}_{3}, \mathrm{CaO}, \mathrm{NiO}$ and $\mathrm{Na}_{2} \mathrm{O}$ contents are given in wt.\%, - = phase is not present or not analyzed. 
Table 5

Selected compositions of crystal inclusions of spinel-group minerals and their hosts

\begin{tabular}{|c|c|c|c|c|c|c|c|c|c|c|}
\hline \multirow{2}{*}{$\begin{array}{l}\text { Sample } \\
\text { Grain } \\
\end{array}$} & \multicolumn{2}{|l|}{ Chk3/4 } & \multicolumn{4}{|l|}{ Chk16 } & \multicolumn{2}{|c|}{ Chk13/1 } & \multicolumn{2}{|c|}{ Chk13/2 } \\
\hline & $9-1$ & $10-1$ & $2-5$ & $1-1$ & $7-1$ & $12-1$ & $6-1$ & $9-1$ & $2-2$ & $17-2$ \\
\hline \multicolumn{11}{|l|}{ Inclusions $^{\mathrm{a}}$} \\
\hline $\mathrm{SiO}_{2}$ & 0.09 & 0.10 & 0.10 & 0.10 & 0.11 & 0.10 & 0.16 & 0.11 & 0.12 & 0.10 \\
\hline $\mathrm{TiO}_{2}$ & 5.18 & 5.98 & 6.96 & 7.11 & 7.05 & 7.43 & 5.78 & 5.99 & 5.70 & 7.84 \\
\hline $\mathrm{Al}_{2} \mathrm{O}_{3}$ & 7.16 & 5.62 & 4.78 & 4.89 & 4.89 & 4.66 & 6.77 & 6.68 & 6.23 & 4.85 \\
\hline $\mathrm{Cr}_{2} \mathrm{O}_{3}$ & 0.16 & 0.09 & 0.05 & 0.08 & 0.05 & 0.16 & 0.08 & 0.10 & 0.06 & 0.05 \\
\hline $\mathrm{Fe}_{2} \mathrm{O}_{3}$ & 51.42 & 51.82 & 51.41 & 50.93 & 50.99 & 49.75 & 51.67 & 50.90 & 52.57 & 49.95 \\
\hline $\mathrm{FeO}$ & 30.03 & 31.11 & 32.44 & 32.72 & 32.57 & 32.68 & 30.89 & 30.71 & 30.94 & 33.54 \\
\hline $\mathrm{MnO}$ & 0.30 & 0.38 & 0.40 & 0.38 & 0.36 & 0.35 & 0.30 & 0.36 & 0.32 & 0.41 \\
\hline $\mathrm{MgO}$ & 4.17 & 3.76 & 3.60 & 3.50 & 3.57 & 3.47 & 4.26 & 4.25 & 4.10 & 3.49 \\
\hline $\mathrm{NiO}$ & 0.01 & 0.09 & 0.01 & 0.05 & 0.03 & 0.11 & 0.02 & 0.04 & 0.01 & 0.04 \\
\hline Total & 98.52 & 98.95 & 99.75 & 99.76 & 99.62 & 98.71 & 99.93 & 99.14 & 100.05 & 100.27 \\
\hline $\mathrm{mg} \#_{\mathrm{sp}}$ & 19.8 & 17.7 & 16.5 & 16.0 & 16.3 & 15.9 & 19.7 & 19.8 & 19.1 & 15.6 \\
\hline $\mathrm{cr}_{\mathrm{sp}}$ & 1.5 & 1.1 & 0.7 & 1.1 & 0.7 & 2.3 & 0.8 & 1.0 & 0.6 & 0.7 \\
\hline $\mathrm{Sp}$ & 15.3 & 12.1 & 10.3 & 10.5 & 10.5 & 10.1 & 14.3 & 14.2 & 13.2 & 10.4 \\
\hline Crt & 0.2 & 0.1 & 0.1 & 0.1 & 0.1 & 0.2 & 0.1 & 0.1 & 0.1 & 0.1 \\
\hline $\mathrm{Mt}$ & 70.3 & 71.3 & 70.6 & 69.9 & 70.1 & 69.0 & 69.9 & 69.3 & 71.3 & 68.2 \\
\hline Usp & 14.2 & 16.4 & 19.1 & 19.5 & 19.4 & 20.6 & 15.6 & 16.3 & 15.4 & 21.4 \\
\hline $\mathrm{Fe}^{2+} / \mathrm{Fe}^{3+}$ & 0.65 & 0.67 & 0.70 & 0.71 & 0.71 & 0.73 & 0.66 & 0.67 & 0.65 & 0.75 \\
\hline Host mineral $^{\mathrm{b}}$ & $\mathrm{Ol}$ & Cpx & $\mathrm{Ol}$ & Opx & Cpx & Pl & $\mathrm{O} 1$ & $\mathrm{Ol}$ & $\mathrm{Ol}$ & Cpx \\
\hline $\mathrm{SiO}_{2}$ & 38.17 & 50.52 & 37.81 & 53.40 & 51.53 & 44.10 & 37.18 & 37.90 & 37.98 & 50.91 \\
\hline $\mathrm{TiO}_{2}$ & 0.03 & 0.49 & $<0.01$ & 0.25 & 0.40 & 0.01 & 0.02 & 0.04 & 0.05 & 0.54 \\
\hline $\mathrm{Al}_{2} \mathrm{O}_{3}$ & 0.03 & 3.28 & 0.03 & 1.75 & 2.53 & 34.01 & 0.02 & 0.04 & 0.02 & 3.02 \\
\hline $\mathrm{Cr}_{2} \mathrm{O}_{3}$ & 0.02 & $<0.01$ & $<0.01$ & $<0.01$ & $<0.01$ & ND & $<0.01$ & 0.01 & $<0.01$ & $<0.01$ \\
\hline $\mathrm{FeO}$ & 22.83 & 8.69 & 23.04 & 16.43 & 8.70 & 0.74 & 24.63 & 23.99 & 23.81 & 9.41 \\
\hline $\mathrm{MnO}$ & 0.44 & 0.25 & 0.43 & 0.54 & 0.37 & 0.01 & 0.39 & 0.46 & 0.42 & 0.34 \\
\hline $\mathrm{MgO}$ & 39.08 & 15.16 & 37.96 & 26.05 & 15.77 & 0.03 & 37.63 & 37.96 & 37.90 & 15.41 \\
\hline $\mathrm{CaO}$ & 0.16 & 20.69 & 0.15 & 1.79 & 19.81 & 18.87 & 0.17 & 0.19 & 0.16 & 19.90 \\
\hline $\mathrm{Na}_{2} \mathrm{O}$ & ND & 0.26 & ND & 0.01 & 0.26 & 0.82 & ND & ND & ND & 0.29 \\
\hline $\mathrm{K}_{2} \mathrm{O}$ & ND & ND & ND & ND & ND & 0.02 & ND & ND & ND & ND \\
\hline $\mathrm{NiO}$ & 0.03 & $<0.01$ & 0.02 & ND & 0.01 & ND & 0.01 & 0.02 & 0.04 & 0.01 \\
\hline Total & 100.79 & 99.34 & 99.44 & 100.22 & 99.38 & 98.61 & 100.05 & 100.61 & 100.38 & 99.83 \\
\hline mg\#, An & 75.3 & 75.7 & 74.6 & 73.9 & 76.4 & 92.6 & 73.1 & 73.8 & 73.9 & 74.5 \\
\hline
\end{tabular}

${ }^{\mathrm{a}}$ Inclusions of spinel-group minerals. $\mathrm{Fe}_{2} \mathrm{O}_{3}$ and $\mathrm{FeO}$ concentrations are calculated assuming spinel stoichiometry, $\mathrm{mg} \#_{\mathrm{sp}}=100 \times \mathrm{Mg} /$ $\left(\mathrm{Mg}+\mathrm{Fe}^{2+}\right), \mathrm{cr}_{\text {sp }}=100 \times \mathrm{Cr} /(\mathrm{Cr}+\mathrm{Al})$ given as atomic ratios. $\mathrm{Sp}=$ spinel $\left[\left(\mathrm{Fe}^{2+}, \mathrm{Mg}\right) \mathrm{Al}_{2} \mathrm{O}_{4}\right], \mathrm{Crt}=$ chromite $\left[\left(\mathrm{Fe}^{2+}, \mathrm{Mg}^{2} \mathrm{Cr}_{2} \mathrm{O}_{4}\right], \mathrm{Mt}^{2}=\mathrm{magnetite}\right.$ $\left[\left(\mathrm{Fe}^{2+}, \mathrm{Mg}\right) \mathrm{Fe}_{2} \mathrm{O}_{4}\right]$ and $\mathrm{Usp}=$ ulvöspinel $\left[\mathrm{Fe}_{2} \mathrm{TiO}_{4}\right]$ are end-member contents given in $\mathrm{mol} \%$. ND=not determined.

${ }^{\mathrm{b}}$ Composition of host mineral. In this table and Table 5, Ol=olivine, $\mathrm{Cpx}=$ clinopyroxene, Opx=orthopyroxene, $\mathrm{Pl}=\mathrm{plagioclase}$; $\mathrm{mg} \#=100 \times \mathrm{Mg} /\left(\mathrm{Mg}+\mathrm{Fe}_{\mathrm{tot}}\right)$, atomic ratio, for olivine, clinopyroxene and orthopyroxene, $\mathrm{An}=\mathrm{mol} \%$ anorthite for plagioclase.

and clinopyroxene hosts have the same rather wide range of An contents $\left(\mathrm{An}_{76-90}\right)$ as the phenocryst compositions. This is surprising because the host $\mathrm{Ol}$ and $\mathrm{Cpx}$ grains have nearly constant compositions, which argues against changes in the major element composition of melt during crystallization (Table 6). We believe the variable An content in plagioclase is related to changing $\mathrm{H}_{2} \mathrm{O}$ contents, a parameter that can strongly effect plagioclase composition (e.g., Sisson and Grove, 1993a) but has no effect on Ol or Cpx $m g$ - number. A few colorless to somewhat bluish Pl crystals of $A_{95-96}$, probably of xenocrystic origin, were noted in sample CHK13/1. They contain inclusions of greenish high-Al spinel and very rare glass inclusions.

The composition of Fe-Ti oxides was determined on grains included in $\mathrm{Ol}, \mathrm{Pl}, \mathrm{Cpx}$ and Opx phenocrysts, as well as those present in the groundmass. The oxides are dominated by magnetite $[\mathrm{Mt}$; $\left.\left(\mathrm{Fe}^{2+}, \mathrm{Mg}\right) \mathrm{Fe}_{2} \mathrm{O}_{4}\right]$, spinel $\left[\mathrm{Sp} ;\left(\mathrm{Fe}^{2+}, \mathrm{Mg}\right) \mathrm{Al}_{2} \mathrm{O}_{4}\right]$ and ulvöspinel [Usp; $\mathrm{Fe}_{2} \mathrm{TiO}_{4}$ ] components, with a full 
Table 6

Compositions of silicate crystal inclusions and their host minerals

\begin{tabular}{|c|c|c|c|c|c|c|c|c|c|c|c|c|}
\hline \multirow{2}{*}{$\frac{\text { Sample }}{\text { Grain }}$} & \multicolumn{2}{|c|}{ CHK3/4 } & \multicolumn{4}{|l|}{ CHK16 } & \multicolumn{6}{|c|}{ CHK13/1 } \\
\hline & $2-1$ & $5-1$ & $3-1$ & $2-1$ & $6-1$ & $7-1$ & $6-2$ & $7-2$ & $1-1$ & $7-1$ & $11-1$ & $13-1$ \\
\hline Inclusions & $\mathrm{Pl}$ & Pl & Opx & $\mathrm{Pl}$ & $\mathrm{Pl}$ & $\mathrm{P} 1$ & Opx & Opx & $\mathrm{P} 1$ & $\mathrm{P} 1$ & Pl & $\mathrm{Pl}$ \\
\hline $\mathrm{SiO}_{2}$ & 46.38 & 45.79 & 53.57 & 46.01 & 46.36 & 46.41 & 53.17 & 53.88 & 48.47 & 45.76 & 47.13 & 47.93 \\
\hline $\mathrm{TiO}_{2}$ & 0.05 & $<0.01$ & 0.22 & 0.01 & 0.02 & 0.02 & 0.17 & 0.24 & 0.03 & 0.02 & 0.01 & 0.02 \\
\hline $\mathrm{Al}_{2} \mathrm{O}_{3}$ & 31.87 & 34.24 & 2.04 & 33.81 & 33.15 & 32.87 & 1.36 & 1.74 & 31.33 & 33.37 & 33.81 & 32.87 \\
\hline $\mathrm{Cr}_{2} \mathrm{O}_{3}$ & ND & ND & $<0.01$ & ND & ND & ND & 0.01 & 0.01 & ND & ND & ND & ND \\
\hline $\mathrm{FeO}$ & 2.03 & 1.79 & 15.54 & 0.71 & 1.06 & 0.99 & 15.89 & 16.60 & 1.36 & 1.31 & 1.37 & 1.44 \\
\hline $\mathrm{MnO}$ & 0.06 & 0.11 & 0.49 & 0.01 & 0.03 & 0.01 & ND & $\mathrm{ND}$ & 0.04 & 0.03 & 0.02 & $<0.01$ \\
\hline $\mathrm{MgO}$ & 0.59 & 0.31 & 26.73 & 0.06 & 0.08 & 0.08 & 27.25 & 25.87 & 0.11 & 0.06 & 0.07 & 0.06 \\
\hline $\mathrm{CaO}$ & 16.85 & 17.44 & 1.70 & 17.92 & 17.44 & 17.32 & 1.65 & 1.93 & 15.42 & 18.46 & 17.32 & 16.59 \\
\hline $\mathrm{Na}_{2} \mathrm{O}$ & 1.58 & 1.29 & ND & 1.47 & 1.64 & 1.74 & ND & ND & 2.67 & 1.15 & 1.73 & 2.08 \\
\hline $\mathrm{K}_{2} \mathrm{O}$ & 0.07 & 0.03 & 0.02 & 0.02 & 0.03 & 0.04 & 0.01 & 0.06 & 0.03 & 0.02 & 0.04 & 0.03 \\
\hline $\mathrm{NiO}$ & ND & ND & 0.07 & ND & ND & ND & 0.01 & $<0.01$ & ND & ND & ND & ND \\
\hline Total & 99.48 & 101.00 & 100.38 & 100.02 & 99.81 & 99.48 & 99.52 & 100.33 & 99.46 & 100.18 & 101.50 & 101.02 \\
\hline mg\#, An & 85.1 & 88.0 & 75.4 & 87.0 & 85.3 & 84.4 & 75.4 & 73.5 & 76.0 & 89.8 & 84.5 & 81.4 \\
\hline Host mineral & $\mathrm{Ol}$ & $\mathrm{Ol}$ & $\mathrm{Ol}$ & $\mathrm{Ol}$ & $\mathrm{Cpx}$ & $\mathrm{Cpx}$ & Cpx & $\mathrm{Cpx}$ & $\mathrm{Ol}$ & $\mathrm{Ol}$ & $\mathrm{Ol}$ & $\mathrm{Ol}$ \\
\hline $\mathrm{SiO}_{2}$ & 38.54 & 38.72 & 37.84 & 37.68 & 50.98 & 51.27 & 51.08 & 51.62 & 37.74 & 37.91 & 37.56 & 37.62 \\
\hline $\mathrm{TiO}_{2}$ & 0.02 & 0.02 & 0.03 & $<0.01$ & 0.44 & 0.37 & 0.40 & 0.38 & 0.01 & $<0.01$ & 0.01 & 0.01 \\
\hline $\mathrm{Al}_{2} \mathrm{O}_{3}$ & 0.01 & 0.02 & 0.01 & 0.03 & 2.57 & 2.30 & 2.21 & 2.52 & 0.02 & 0.01 & 0.03 & 0.03 \\
\hline $\mathrm{Cr}_{2} \mathrm{O}_{3}$ & $<0.01$ & 0.02 & 0.01 & 0.01 & 0.02 & 0.02 & $<0.01$ & 0.01 & $<0.01$ & $<0.01$ & 0.01 & 0.01 \\
\hline $\mathrm{FeO}$ & 24.44 & 24.15 & 24.04 & 26.08 & 8.81 & 8.64 & 9.10 & 8.98 & 23.49 & 24.35 & 24.46 & 24.18 \\
\hline $\mathrm{MnO}$ & 0.55 & 0.53 & 0.55 & 0.57 & 0.33 & 0.32 & $\mathrm{ND}$ & $\mathrm{ND}$ & 0.51 & 0.44 & 0.46 & 0.45 \\
\hline $\mathrm{MgO}$ & 36.99 & 37.08 & 37.63 & 35.91 & 15.44 & 15.61 & 16.30 & 15.77 & 38.01 & 37.82 & 37.89 & 37.75 \\
\hline $\mathrm{CaO}$ & 0.08 & 0.09 & 0.19 & 0.14 & 19.91 & 19.68 & 19.75 & 20.11 & 0.09 & 0.10 & 0.16 & 0.17 \\
\hline $\mathrm{Na}_{2} \mathrm{O}$ & ND & ND & ND & ND & 0.25 & 0.25 & 0.19 & 0.28 & ND & ND & ND & ND \\
\hline $\mathrm{NiO}$ & 0.01 & 0.02 & 0.09 & 0.06 & 0.03 & $<0.01$ & 0.01 & 0.01 & 0.01 & 0.01 & 0.03 & $<0.01$ \\
\hline Total & 100.64 & 100.65 & 100.39 & 100.48 & 98.78 & 98.46 & 99.04 & 99.68 & 99.88 & 100.64 & 100.61 & 100.22 \\
\hline mg\#, An & 73.0 & 73.2 & 73.6 & 71.1 & 75.8 & 76.3 & 76.2 & 75.8 & 74.3 & 73.5 & 73.4 & 73.6 \\
\hline
\end{tabular}

For explanations see captions for Table 5 .

compositional range being $\mathrm{Mt}_{68-74} \mathrm{Sp}_{10-16} \mathrm{Usp}_{12-21}$. The chromite $\left[\mathrm{Crt} ;\left(\mathrm{Fe}^{2+}, \mathrm{Mg}\right) \mathrm{Cr}_{2} \mathrm{O}_{4}\right]$ component is negligible (0.1-0.3 mol\%; Table 5). The $\mathrm{Fe}^{2+} / \mathrm{Fe}^{3+}$ ratios calculated from spinel stoichiometry are from 0.6 to 0.7 and show no relation to the age of eruption.

\section{Glass composition}

\subsection{Major elements}

The compositions of glassy melt inclusions in $\mathrm{Ol}$, Cpx, Opx and Pl hosts, together with bulk-rock and groundmass glass compositions are given in Table 7 and important features are illustrated in Figs. 4 and 5. Before interpreting these data it is necessary to discuss corrections for changes in composition after trapping by equilibration with, or crystallization of, the host minerals during cooling. It is well known that melt inclusions in olivine can lose significant amounts of $\mathrm{Fe}$ after trapping due to $\mathrm{Fe}-\mathrm{Mg}$ exchange with the host followed by diffusion of $\mathrm{Fe}$ through the Ol matrix (e.g., Sobolev, 1983; Gurenko et al., 1991; Danyushevsky et al., 2000). Similar iron loss could also be observed in Cpx-hosted inclusions but the effect is weaker, probably due to the lower rate of $\mathrm{Fe}-\mathrm{Mg}$ inter-diffusion in clinopyroxene than in olivine (Chakraborty, 1997; Dimanov and Sautter, 2000; Azough and Freer, 2000). For inclusions in $\mathrm{Pl}$, the effect is negligible since there is nearly no capacity for $\mathrm{Fe}-\mathrm{Mg}$ exchange with the host mineral. For the samples analyzed in this study, the $\mathrm{FeO}$ contents of inclusions in $\mathrm{Ol}, \mathrm{Cpx}, \mathrm{Opx}$ and Pl host minerals are all very similar, so we conclude 
Table 7

Major elements, $\mathrm{H}_{2} \mathrm{O}, \mathrm{S}$ and $\mathrm{Cl}$ contents in glass inclusions

\begin{tabular}{|c|c|c|c|c|c|c|c|c|c|c|c|c|c|}
\hline \multirow{2}{*}{$\frac{\text { Sample }}{\text { Grain No }}$} & \multicolumn{10}{|c|}{ CHK3/4 } & \multicolumn{3}{|c|}{ CHK13/1 } \\
\hline & $1-1$ & $2-1$ & $4-1$ & $9-1$ & $18-1$ & $10-1$ & $11-1$ & $13-1$ & $14-1$ & $16-3$ & $1-1$ & $1-2$ & $2-1$ \\
\hline \multicolumn{14}{|c|}{ Glass inclusions $^{\mathrm{a}}$} \\
\hline $\mathrm{SiO}_{2}$ & 52.12 & 55.13 & 51.12 & 53.23 & 52.51 & 54.75 & 54.77 & 55.16 & 51.41 & 52.55 & 52.17 & 51.57 & 51.31 \\
\hline $\mathrm{TiO}_{2}$ & 1.34 & 1.00 & 0.50 & 1.26 & 0.98 & 1.00 & 1.08 & 0.73 & 0.84 & 0.95 & 0.98 & 0.99 & 0.93 \\
\hline $\mathrm{Al}_{2} \mathrm{O}_{3}$ & 16.72 & 15.76 & 16.46 & 16.70 & 15.98 & 16.26 & 14.84 & 17.01 & 16.79 & 16.45 & 17.40 & 16.45 & 16.54 \\
\hline $\mathrm{FeO}_{\text {tot }}$ & 9.89 & 10.06 & 11.32 & 9.83 & 10.05 & 8.81 & 11.07 & 7.78 & 9.51 & 8.84 & 10.87 & 10.96 & 11.21 \\
\hline $\mathrm{MnO}$ & 0.20 & 0.23 & 0.25 & 0.21 & 0.24 & 0.25 & 0.36 & 0.18 & 0.26 & 0.19 & 0.24 & 0.22 & 0.23 \\
\hline $\mathrm{MgO}$ & 4.52 & 4.20 & 4.87 & 4.11 & 4.14 & 3.50 & 3.51 & 3.22 & 4.13 & 3.60 & 4.21 & 4.62 & 4.94 \\
\hline $\mathrm{CaO}$ & 8.13 & 7.83 & 9.61 & 8.47 & 8.38 & 7.46 & 7.03 & 7.73 & 8.09 & 8.02 & 9.07 & 8.74 & 8.64 \\
\hline $\mathrm{Na}_{2} \mathrm{O}$ & 2.61 & 2.74 & 2.25 & 2.66 & 3.00 & 3.24 & 3.07 & 3.31 & 2.51 & 2.56 & 3.11 & 2.45 & 2.60 \\
\hline $\mathrm{K}_{2} \mathrm{O}$ & 0.80 & 0.77 & 0.40 & 0.80 & 0.76 & 0.88 & 0.75 & 0.99 & 0.78 & 0.90 & 0.76 & 0.67 & 0.71 \\
\hline $\mathrm{P}_{2} \mathrm{O}_{5}$ & 0.27 & 0.26 & 0.18 & 0.28 & 0.21 & 0.22 & 0.22 & 0.22 & 0.17 & 0.21 & 0.19 & 0.20 & 0.22 \\
\hline Total & 96.60 & 97.98 & 96.96 & 97.55 & 96.25 & 96.37 & 96.70 & 96.33 & 94.49 & 94.27 & 99.00 & 96.87 & 97.33 \\
\hline S & 0.15 & 0.13 & 0.29 & 0.15 & 0.12 & 0.10 & 0.11 & 0.08 & 0.18 & 0.11 & 0.17 & 0.13 & 0.15 \\
\hline $\mathrm{Cl}$ & 0.09 & 0.10 & 0.08 & 0.09 & 0.08 & 0.10 & 0.10 & 0.09 & 0.11 & 0.09 & 0.10 & 0.10 & 0.09 \\
\hline $\mathrm{H}_{2} \mathrm{O}_{\text {calc }}$ & 3.2 & 1.8 & 2.7 & 2.2 & 3.6 & 3.4 & 3.1 & 3.5 & 5.2 & 5.5 & 0.7 & 2.9 & 2.4 \\
\hline
\end{tabular}

\begin{tabular}{|c|c|c|c|c|c|c|c|c|c|c|c|c|c|}
\hline Host mineral $^{\mathrm{b}}$ & $\mathrm{Ol}$ & $\mathrm{Ol}$ & $\mathrm{Ol}$ & $\mathrm{Ol}$ & $\mathrm{Ol}$ & $\mathrm{Cpx}$ & Cpx & Pl & $\mathrm{Pl}$ & $\mathrm{Pl}$ & $\mathrm{O} 1$ & $\mathrm{Ol}$ & $\mathrm{Ol}$ \\
\hline $\mathrm{SiO}_{2}$ & 38.17 & 37.52 & 37.88 & 38.17 & 37.77 & 50.47 & 51.28 & 46.07 & 45.54 & 47.53 & 37.74 & 38.06 & 38.55 \\
\hline $\mathrm{TiO}_{2}$ & 0.02 & 0.02 & 0.01 & 0.02 & 0.01 & 0.50 & 0.50 & 0.02 & 0.08 & 0.17 & 0.01 & 0.03 & 0.01 \\
\hline $\mathrm{Al}_{2} \mathrm{O}_{3}$ & 0.02 & 0.03 & 0.02 & 0.02 & 0.06 & 3.60 & 2.21 & 33.87 & 32.19 & 30.83 & 0.02 & 0.02 & 0.03 \\
\hline $\mathrm{Cr}_{2} \mathrm{O}_{3}$ & $\mathrm{BDL}$ & $\mathrm{BDL}$ & $\mathrm{BDL}$ & 0.01 & $\mathrm{BDL}$ & 0.01 & 0.01 & ND & ND & ND & $\mathrm{BDL}$ & $\mathrm{BDL}$ & 0.01 \\
\hline $\mathrm{FeO}_{\text {tot }}$ & 23.22 & 25.25 & 24.10 & 23.04 & 24.39 & 8.01 & 10.68 & 0.74 & 1.48 & 1.98 & 23.49 & 23.72 & 23.69 \\
\hline $\mathrm{MnO}$ & 0.43 & 0.57 & 0.49 & 0.49 & 0.55 & 0.22 & 0.51 & 0.01 & 0.03 & 0.05 & 0.51 & 0.50 & 0.47 \\
\hline $\mathrm{MgO}$ & 38.94 & 37.20 & 37.42 & 39.00 & 37.39 & 15.00 & 15.43 & 0.06 & 0.37 & 0.58 & 38.01 & 38.23 & 37.88 \\
\hline $\mathrm{CaO}$ & 0.16 & 0.16 & 0.25 & 0.16 & 0.17 & 21.30 & 18.70 & 18.17 & 17.54 & 16.22 & 0.16 & 0.20 & 0.18 \\
\hline $\mathrm{Na}_{2} \mathrm{O}$ & ND & ND & ND & ND & ND & 0.24 & 0.31 & 1.34 & 1.33 & 1.89 & ND & ND & ND \\
\hline $\mathrm{K}_{2} \mathrm{O}$ & ND & ND & ND & ND & ND & ND & ND & 0.02 & 0.06 & 0.15 & ND & ND & ND \\
\hline $\mathrm{NiO}$ & 0.02 & BDL & 0.04 & 0.04 & 0.03 & 0.01 & 0.01 & ND & ND & ND & 0.01 & BDL & 0.04 \\
\hline Total & 100.98 & 100.75 & 100.21 & 100.95 & 100.37 & 99.36 & 99.64 & 100.30 & 98.62 & 99.40 & 99.95 & 100.76 & 100.86 \\
\hline mg\#, An & 74.9 & 72.4 & 73.5 & 75.1 & 73.2 & 76.9 & 72.0 & 88.1 & 87.6 & 81.8 & 74.3 & 74.2 & 74.0 \\
\hline $\mathrm{K}_{\mathrm{D}}$ & 0.273 & 0.283 & 0.277 & 0.247 & 0.269 & 0.212 & 0.219 & 5.82 & 4.11 & 2.75 & 0.239 & 0.262 & 0.276 \\
\hline
\end{tabular}

\begin{tabular}{|c|c|c|c|c|c|c|c|c|c|c|c|c|c|}
\hline \multicolumn{14}{|c|}{ Corrected compositions $^{\mathrm{c}}$} \\
\hline $\mathrm{SiO}_{2}$ & 52.1 & 55.1 & 51.1 & 53.0 & 52.5 & 54.4 & 54.5 & 55.2 & 51.5 & 52.6 & 51.9 & 51.4 & 51.3 \\
\hline $\mathrm{TiO}_{2}$ & 1.3 & 1.0 & 0.5 & 1.2 & 1.0 & 1.0 & 1.1 & 0.7 & 0.8 & 0.9 & 1.0 & 1.0 & 0.9 \\
\hline $\mathrm{Al}_{2} \mathrm{O}_{3}$ & 16.7 & 15.7 & 16.4 & 16.4 & 16.0 & 15.5 & 14.4 & 17.0 & 16.8 & 16.5 & 17.1 & 16.2 & 16.5 \\
\hline $\mathrm{Fe}_{2} \mathrm{O}_{3}$ & 2.7 & 2.6 & 2.7 & 2.7 & 2.7 & 2.1 & 3.3 & 1.6 & 1.9 & 1.7 & 3.2 & 2.8 & 2.9 \\
\hline $\mathrm{FeO}$ & 7.5 & 7.7 & 8.9 & 7.6 & 7.6 & 6.9 & 8.1 & 6.4 & 7.8 & 7.3 & 8.2 & 8.6 & 8.6 \\
\hline $\mathrm{MnO}$ & 0.2 & 0.2 & 0.3 & 0.2 & 0.2 & 0.2 & 0.4 & 0.2 & 0.3 & 0.2 & 0.2 & 0.2 & 0.2 \\
\hline $\mathrm{MgO}$ & 4.5 & 4.3 & 5.0 & 4.6 & 4.1 & 4.3 & 4.0 & 3.2 & 4.1 & 3.6 & 4.7 & 5.0 & 4.9 \\
\hline $\mathrm{CaO}$ & 8.1 & 7.8 & 9.6 & 8.3 & 8.4 & 8.2 & 7.5 & 7.7 & 8.1 & 8.0 & 8.9 & 8.6 & 8.6 \\
\hline $\mathrm{Na}_{2} \mathrm{O}$ & 2.6 & 2.7 & 2.2 & 2.6 & 3.0 & 3.0 & 2.9 & 3.3 & 2.5 & 2.6 & 3.1 & 2.4 & 2.6 \\
\hline $\mathrm{K}_{2} \mathrm{O}$ & 0.8 & 0.8 & 0.4 & 0.8 & 0.8 & 0.8 & 0.7 & 1.0 & 0.8 & 0.9 & 0.7 & 0.7 & 0.7 \\
\hline $\mathrm{P}_{2} \mathrm{O}_{5}$ & 0.3 & 0.3 & 0.2 & 0.3 & 0.2 & 0.2 & 0.2 & 0.2 & 0.2 & 0.2 & 0.2 & 0.2 & 0.2 \\
\hline $\mathrm{H}_{2} \mathrm{O}$ & 3.2 & 1.8 & 2.7 & 2.2 & 3.6 & 3.2 & 2.9 & 3.5 & 5.2 & 5.5 & 0.7 & 2.9 & 2.4 \\
\hline $\mathrm{S}$ & 0.2 & 0.1 & 0.3 & 0.1 & 0.1 & 0.1 & 0.1 & 0.1 & 0.2 & 0.1 & 0.2 & 0.1 & 0.2 \\
\hline $\mathrm{Cl}$ & 0.1 & 0.1 & 0.1 & 0.1 & 0.1 & 0.1 & 0.1 & 0.1 & 0.1 & 0.1 & 0.1 & 0.1 & 0.1 \\
\hline$P_{\text {cryst-melt }}, \mathrm{MPa}^{\mathrm{d}}$ & 330 & 200 & 0.1 & 240 & 80 & 90 & 130 & 0.1 & 150 & 0.1 & 230 & 240 & 270 \\
\hline$T_{\text {cryst-melt }},{ }^{\circ} \mathrm{C}^{\mathrm{d}}$ & 1018 & 1031 & 1003 & 1028 & 989 & 1008 & 1004 & 970 & 939 & 908 & 1055 & 1027 & 1032 \\
\hline$T_{\mathrm{S} \& \mathrm{G} 93},{ }^{\circ} \mathrm{C}^{\mathrm{d}}$ & 1077 & 1096 & 1086 & 1102 & 1039 & 1048 & 1038 & 1032 & 1001 & 974 & 1154 & 1085 & 1101 \\
\hline$T_{\text {mean }},{ }^{\circ} \mathrm{C}^{\mathrm{d}}$ & 1048 & 1064 & 1045 & 1065 & 1014 & 1028 & 1021 & 1001 & 970 & 941 & 1105 & 1056 & 1067 \\
\hline Host-add $(\%)^{\mathrm{e}}$ & 0 & 0.3 & 0.3 & 1.5 & 0 & 7.2 & 4.6 & 0 & 0 & 0 & 1.4 & 1.3 & 0 \\
\hline
\end{tabular}




\begin{tabular}{|c|c|c|c|c|c|c|c|c|c|c|c|}
\hline \multirow[b]{2}{*}{$4-1$} & \multirow[b]{2}{*}{$9-1$} & \multirow[b]{2}{*}{$10-1$} & \multirow[b]{2}{*}{$11-1$} & \multirow[b]{2}{*}{$19-1$} & \multirow[b]{2}{*}{$19-2$} & \multirow[b]{2}{*}{$18-1$} & \multicolumn{5}{|c|}{ CHK13/2 } \\
\hline & & & & & & & $10-1$ & $12-1$ & $12-3$ & $14-2$ & $20-1$ \\
\hline 53.79 & 51.38 & 50.85 & 53.08 & 53.00 & 53.76 & 56.35 & 51.80 & 54.74 & 55.17 & 53.77 & 51.41 \\
\hline 1.47 & 0.95 & 0.99 & 1.12 & 1.03 & 1.03 & 0.97 & 0.94 & 0.82 & 1.29 & 1.09 & 0.88 \\
\hline 16.25 & 16.76 & 16.16 & 17.58 & 15.64 & 15.92 & 14.47 & 16.62 & 15.95 & 13.66 & 15.72 & 16.32 \\
\hline 10.64 & 11.05 & 10.95 & 10.54 & 10.27 & 9.78 & 10.21 & 10.22 & 8.59 & 11.57 & 9.28 & 9.08 \\
\hline 0.22 & 0.23 & 0.24 & 0.25 & 0.21 & 0.24 & 0.22 & 0.25 & 0.23 & 0.30 & 0.18 & 0.19 \\
\hline 3.29 & 4.24 & 4.80 & 3.99 & 4.22 & 3.88 & 4.20 & 4.29 & 3.89 & 4.57 & 4.47 & 4.23 \\
\hline 8.40 & 8.92 & 8.45 & 8.81 & 8.11 & 7.89 & 6.62 & 8.30 & 7.12 & 7.59 & 7.54 & 7.45 \\
\hline 2.27 & 2.65 & 2.75 & 3.29 & 2.87 & 3.03 & 2.50 & 2.89 & 2.35 & 3.14 & 2.90 & 2.72 \\
\hline 0.86 & 0.62 & 0.69 & 0.83 & 0.80 & 0.82 & 1.23 & 0.78 & 1.01 & 1.08 & 0.97 & 0.96 \\
\hline 0.30 & 0.20 & 0.20 & 0.24 & 0.24 & 0.26 & 0.19 & 0.21 & 0.17 & 0.26 & 0.24 & 0.21 \\
\hline 97.49 & 97.00 & 96.08 & 99.73 & 96.39 & 96.61 & 96.96 & 96.30 & 94.87 & 98.63 & 96.16 & 93.45 \\
\hline 0.07 & 0.18 & 0.13 & 0.12 & 0.06 & 0.07 & 0.04 & 0.15 & 0.12 & 0.04 & 0.09 & 0.1 \\
\hline 0.11 & 0.10 & 0.09 & 0.12 & 0.10 & 0.11 & 0.13 & 0.11 & 0.10 & 0.13 & 0.11 & 0.1 \\
\hline 2.3 & 2.7 & 3.7 & 0.0 & 3.5 & 3.2 & 2.9 & 3.4 & 4.9 & 1.2 & 3.6 & 6.4 \\
\hline
\end{tabular}

\begin{tabular}{llllllllllll} 
Ol & Ol & Ol & Ol & Ol & Ol & Pl & Ol & Pl & Pl & Pl & Pl \\
\hline 38.61 & 37.59 & 37.64 & 37.47 & 37.40 & 37.40 & 46.91 & 37.85 & 46.15 & 46.28 & 45.33 & 45.87 \\
BDL & 0.01 & 0.01 & 0.01 & 0.02 & 0.02 & 0.01 & 0.01 & 0.03 & 0.03 & 0.03 & 0.02 \\
0.01 & 0.02 & 0.03 & 0.03 & 0.02 & 0.02 & 33.42 & 0.02 & 33.85 & 33.72 & 34.21 & 33.38 \\
BDL & 0.01 & BDL & 0.01 & 0.02 & 0.02 & ND & BDL & ND & ND & ND & ND \\
24.24 & 24.33 & 24.28 & 24.42 & 24.58 & 24.58 & 0.70 & 23.88 & 0.62 & 0.66 & 0.68 & 0.68 \\
0.44 & 0.47 & 0.46 & 0.49 & 0.45 & 0.45 & ND & 0.40 & BDL & 0.01 & BDL & BDL \\
37.41 & 37.57 & 37.39 & 37.73 & 37.32 & 37.32 & 0.08 & 37.60 & 0.07 & 0.07 & 0.05 & 0.09 \\
0.18 & 0.17 & 0.18 & 0.17 & 0.16 & 0.16 & 16.95 & 0.13 & 17.91 & 17.85 & 18.25 & 17.97 \\
ND & ND & ND & ND & ND & ND & 1.82 & ND & 1.38 & 1.46 & 1.20 & 1.43 \\
ND & ND & ND & ND & ND & ND & 0.04 & ND & 0.02 & 0.03 & 0.03 & 0.03 \\
0.01 & BDL & 0.04 & 0.04 & 0.01 & 0.01 & ND & BDL & ND & ND & ND & ND \\
100.90 & 100.17 & 100.03 & 100.37 & 99.98 & 99.98 & 99.93 & 99.89 & 100.03 & 100.11 & 99.78 & 99.47 \\
73.3 & 73.4 & 73.3 & 73.4 & 73.0 & 73.0 & 83.5 & 73.7 & 87.7 & 87.0 & 89.2 & 87.3 \\
0.200 & 0.248 & 0.285 & 0.245 & 0.271 & 0.261 & 3.51 & 0.267 & 4.28 & 4.30 & 5.87 & 4.58
\end{tabular}

\begin{tabular}{|c|c|c|c|c|c|c|c|c|c|c|c|}
\hline 52.8 & 51.1 & 50.8 & 53.0 & 52.9 & 53.6 & 56.3 & 51.8 & 54.8 & 55.1 & 53.8 & 51.4 \\
\hline 1.4 & 0.9 & 1.0 & 1.1 & 1.0 & 1.0 & 1.0 & 0.9 & 0.8 & 1.3 & 1.1 & 0.9 \\
\hline 15.3 & 16.5 & 16.2 & 17.5 & 15.6 & 15.8 & 14.5 & 16.6 & 16.0 & 13.6 & 15.7 & 16.3 \\
\hline 3.1 & 3.0 & 2.9 & 3.2 & 2.6 & 2.5 & 2.5 & 2.6 & 1.5 & 3.7 & 2.1 & 1.8 \\
\hline 8.7 & 8.6 & 8.3 & 7.7 & 7.7 & 7.9 & 7.9 & 7.9 & 7.3 & 8.3 & 7.4 & 7.5 \\
\hline 0.2 & 0.2 & 0.2 & 0.2 & 0.2 & 0.2 & 0.2 & 0.2 & 0.2 & 0.3 & 0.2 & 0.2 \\
\hline 5.0 & 4.8 & 4.8 & 4.2 & 4.3 & 4.1 & 4.2 & 4.4 & 3.9 & 4.6 & 4.5 & 4.2 \\
\hline 7.9 & 8.8 & 8.4 & 8.8 & 8.1 & 7.8 & 6.6 & 8.3 & 7.1 & 7.6 & 7.5 & 7.5 \\
\hline 2.1 & 2.6 & 2.7 & 3.3 & 2.9 & 3.0 & 2.5 & 2.9 & 2.4 & 3.1 & 2.9 & 2.7 \\
\hline 0.8 & 0.6 & 0.7 & 0.8 & 0.8 & 0.8 & 1.2 & 0.8 & 1.0 & 1.1 & 1.0 & 1.0 \\
\hline 0.3 & 0.2 & 0.2 & 0.2 & 0.2 & 0.3 & 0.2 & 0.2 & 0.2 & 0.3 & 0.2 & 0.2 \\
\hline 2.2 & 2.7 & 3.7 & 0.0 & 3.5 & 3.2 & 2.9 & 3.4 & 4.9 & 1.2 & 3.6 & 6.4 \\
\hline 0.1 & 0.2 & 0.1 & 0.1 & 0.1 & 0.1 & 0.0 & 0.2 & 0.1 & 0.0 & 0.1 & 0.1 \\
\hline 0.1 & 0.1 & 0.1 & 0.1 & 0.1 & 0.1 & 0.1 & 0.1 & 0.1 & 0.1 & 0.1 & 0.1 \\
\hline 330 & 190 & 280 & 170 & 150 & 190 & 420 & 230 & 240 & 250 & 380 & 380 \\
\hline 1046 & 1018 & 1017 & 1078 & 1002 & 1006 & 1003 & 1006 & 950 & 1035 & 993 & 931 \\
\hline 1098 & 1089 & 1056 & 1168 & 1042 & 1051 & 1051 & 1059 & 998 & 1092 & 1048 & 970 \\
\hline 1072 & 1054 & 1037 & 1123 & 1022 & 1029 & 1027 & 1033 & 974 & 1064 & 1021 & 951 \\
\hline 5.4 & 1.7 & 0 & 0.5 & 0.2 & 0.1 & 0 & 0.4 & 0 & 0 & 0 & 0 \\
\hline
\end{tabular}


Table 7 (continued)

\begin{tabular}{|c|c|c|c|c|c|c|c|c|c|c|c|c|c|c|}
\hline \multirow{2}{*}{$\frac{\text { Sample }}{\text { Grain }}$} & \multicolumn{10}{|l|}{ CHK16 } & \multicolumn{4}{|c|}{ Groundmass glasses } \\
\hline & $2-1$ & $2-2$ & $3-1$ & $6-1$ & $13-1$ & $13-2$ & $13-3$ & $14-2$ & $15-1$ & $15-2$ & 3/4Gl & 13/1Gl & $13 / 2 \mathrm{Gl}$ & $16 \mathrm{Gl}$ \\
\hline \multicolumn{15}{|l|}{ Glass inclusions $^{\mathrm{a}}$} \\
\hline $\mathrm{SiO}_{2}$ & 54.88 & 56.09 & 50.43 & 57.19 & 55.06 & 53.00 & 53.95 & 55.61 & 58.49 & 57.91 & 55.75 & 55.16 & 58.28 & 57.32 \\
\hline $\mathrm{TiO}_{2}$ & 1.10 & 0.99 & 1.09 & 0.88 & 1.22 & 1.04 & 0.97 & 0.93 & 0.81 & 0.87 & 1.13 & 1.13 & 1.18 & 1.00 \\
\hline $\mathrm{Al}_{2} \mathrm{O}_{3}$ & 16.64 & 16.31 & 17.81 & 16.87 & 13.45 & 15.85 & 15.62 & 16.25 & 16.38 & 15.56 & 15.18 & 16.76 & 15.63 & 16.28 \\
\hline $\mathrm{FeO}_{\text {tot }}$ & 8.87 & 9.64 & 9.05 & 7.02 & 12.05 & 9.71 & 8.89 & 8.10 & 7.04 & 7.48 & 10.54 & 9.66 & 8.84 & 9.11 \\
\hline $\mathrm{MnO}$ & 0.29 & 0.21 & 0.26 & 0.17 & 0.33 & 0.25 & 0.23 & 0.24 & 0.19 & 0.19 & 0.26 & 0.24 & 0.23 & 0.25 \\
\hline $\mathrm{MgO}$ & 3.87 & 3.59 & 2.14 & 2.35 & 5.33 & 4.08 & 3.75 & 3.32 & 2.76 & 3.05 & 4.11 & 2.94 & 2.63 & 3.32 \\
\hline $\mathrm{CaO}$ & 7.67 & 8.21 & 10.63 & 6.05 & 6.75 & 7.24 & 7.55 & 7.20 & 6.57 & 6.53 & 7.77 & 9.00 & 8.12 & 7.26 \\
\hline $\mathrm{Na}_{2} \mathrm{O}$ & 3.11 & 2.93 & 2.33 & 3.63 & 3.28 & 2.87 & 2.67 & 2.70 & 3.18 & 3.24 & 2.86 & 2.96 & 3.18 & 3.39 \\
\hline $\mathrm{K}_{2} \mathrm{O}$ & 0.85 & 1.13 & 0.51 & 1.42 & 1.16 & 1.11 & 1.09 & 1.16 & 1.42 & 1.36 & 0.94 & 0.94 & 1.09 & 1.13 \\
\hline $\mathrm{P}_{2} \mathrm{O}_{5}$ & 0.26 & 0.27 & 0.16 & 0.27 & 0.32 & 0.22 & 0.22 & 0.26 & 0.22 & 0.26 & 0.27 & 0.24 & 0.26 & 0.27 \\
\hline Total & 97.54 & 99.37 & 94.41 & 95.85 & 98.95 & 95.37 & 94.94 & 95.77 & 97.06 & 96.45 & 98.81 & 99.03 & 99.44 & 99.33 \\
\hline S & 0.11 & 0.13 & 0.28 & 0.15 & 0.09 & 0.16 & 0.17 & 0.13 & 0.10 & 0.12 & 0.01 & 0.01 & 0.01 & 0.02 \\
\hline $\mathrm{Cl}$ & 0.10 & 0.10 & 0.08 & 0.11 & 0.13 & 0.11 & 0.13 & 0.10 & 0.08 & 0.10 & 0.08 & 0.09 & 0.09 & 0.08 \\
\hline $\mathrm{H}_{2} \mathrm{O}_{\text {calc }}$ & 2.2 & 0.4 & 5.2 & 3.9 & 0.8 & 4.4 & 4.8 & 4.0 & 2.8 & 3.3 & 1.1 & 0.9 & 0.5 & 0.6 \\
\hline Host mineral $^{\mathrm{b}}$ & $\mathrm{Ol}$ & $\mathrm{Ol}$ & $\mathrm{Ol}$ & Cpx & $\mathrm{Pl}$ & Pl & Pl & Pl & Pl & Pl & - & - & - & - \\
\hline $\mathrm{SiO}_{2}$ & 37.68 & 37.68 & 37.81 & 50.84 & 46.33 & 46.43 & 46.43 & 46.45 & 47.25 & 47.17 & - & - & - & - \\
\hline $\mathrm{TiO}_{2}$ & BDL & BDL & BDL & 0.45 & 0.02 & BDL & 0.01 & 0.03 & 0.01 & 0.02 & - & - & - & - \\
\hline $\mathrm{Al}_{2} \mathrm{O}_{3}$ & 0.03 & 0.03 & 0.03 & 2.60 & 33.98 & 33.76 & 33.51 & 33.40 & 32.79 & 32.85 & - & - & - & - \\
\hline $\mathrm{Cr}_{2} \mathrm{O}_{3}$ & 0.01 & 0.01 & BDL & 0.01 & ND & ND & ND & ND & ND & ND & - & - & - & - \\
\hline $\mathrm{FeO}_{\text {tot }}$ & 26.08 & 26.08 & 23.04 & 9.28 & 0.68 & 0.66 & 0.71 & 0.73 & 0.73 & 0.72 & - & - & - & - \\
\hline $\mathrm{MnO}$ & 0.57 & 0.57 & 0.43 & 0.37 & 0.01 & BDL & ND & ND & ND & ND & - & - & - & - \\
\hline $\mathrm{MgO}$ & 35.91 & 35.91 & 37.96 & 15.59 & 0.07 & 0.07 & 0.07 & 0.07 & 0.08 & 0.08 & - & - & - & - \\
\hline $\mathrm{CaO}$ & 0.16 & 0.16 & 0.15 & 19.33 & 17.86 & 17.78 & 17.31 & 17.13 & 16.65 & 16.78 & - & - & - & - \\
\hline $\mathrm{Na}_{2} \mathrm{O}$ & ND & ND & ND & 0.24 & 1.43 & 1.49 & 1.63 & 1.68 & 1.95 & 1.96 & - & - & - & - \\
\hline $\mathrm{K}_{2} \mathrm{O}$ & ND & ND & ND & ND & 0.02 & 0.04 & 0.04 & 0.04 & 0.05 & 0.04 & - & - & - & - \\
\hline $\mathrm{NiO}$ & 0.06 & 0.06 & 0.02 & 0.04 & ND & ND & ND & ND & ND & ND & - & - & - & - \\
\hline Total & 100.50 & 100.50 & 99.44 & 98.75 & 100.40 & 100.23 & 99.71 & 99.53 & 99.51 & 99.62 & - & - & - & - \\
\hline mg\#, An & 71.1 & 71.1 & 74.6 & 75.0 & 87.2 & 86.6 & 85.2 & 84.7 & 82.3 & 82.4 & - & - & - & - \\
\hline $\mathrm{K}_{\mathrm{D}}$ & 0.317 & 0.270 & 0.144 & 0.199 & 6.09 & 4.75 & 3.76 & 3.83 & 4.13 & 4.25 & - & - & - & - \\
\hline \multicolumn{15}{|c|}{ Corrected compositions $^{\mathrm{c}}$} \\
\hline $\mathrm{SiO}_{2}$ & 54.9 & 56.1 & 49.3 & 56.5 & 55.0 & 53.0 & 54.0 & 53.8 & 58.5 & 58.0 & 55.6 & 55.1 & 58.2 & 57.2 \\
\hline $\mathrm{TiO}_{2}$ & 1.1 & 1.0 & 1.0 & 0.9 & 1.2 & 1.0 & 1.0 & 1.1 & 0.8 & 0.9 & 1.1 & 1.1 & 1.2 & 1.0 \\
\hline $\mathrm{Al}_{2} \mathrm{O}_{3}$ & 16.6 & 16.3 & 16.2 & 15.9 & 13.4 & 15.9 & 15.6 & 15.7 & 16.4 & 15.6 & 15.1 & 16.7 & 15.6 & 16.3 \\
\hline $\mathrm{Fe}_{2} \mathrm{O}_{3}$ & 2.0 & 2.4 & 2.4 & 1.5 & 3.8 & 2.3 & 1.7 & 2.0 & 1.3 & 1.5 & 2.9 & 2.6 & 2.3 & 2.4 \\
\hline $\mathrm{FeO}$ & 7.0 & 7.4 & 8.7 & 6.0 & 8.6 & 7.7 & 7.3 & 7.5 & 5.8 & 6.2 & 7.9 & 7.3 & 6.8 & 6.9 \\
\hline $\mathrm{MnO}$ & 0.3 & 0.2 & 0.2 & 0.2 & 0.3 & 0.3 & 0.2 & 0.2 & 0.2 & 0.2 & 0.3 & 0.2 & 0.2 & 0.3 \\
\hline $\mathrm{MgO}$ & 3.9 & 3.6 & 5.0 & 3.5 & 5.3 & 4.1 & 3.8 & 4.5 & 2.8 & 3.1 & 4.1 & 2.9 & 2.6 & 3.3 \\
\hline $\mathrm{CaO}$ & 7.7 & 8.2 & 9.7 & 7.2 & 6.7 & 7.2 & 7.6 & 7.5 & 6.6 & 6.5 & 7.8 & 9.0 & 8.1 & 7.2 \\
\hline $\mathrm{Na}_{2} \mathrm{O}$ & 3.1 & 2.9 & 2.1 & 3.3 & 3.3 & 2.9 & 2.7 & 2.9 & 3.2 & 3.2 & 2.9 & 3.0 & 3.2 & 3.4 \\
\hline $\mathrm{K}_{2} \mathrm{O}$ & 0.9 & 1.1 & 0.5 & 1.3 & 1.2 & 1.1 & 1.1 & 1.0 & 1.4 & 1.4 & 0.9 & 0.9 & 1.1 & 1.1 \\
\hline $\mathrm{P}_{2} \mathrm{O}_{5}$ & 0.3 & 0.3 & 0.1 & 0.2 & 0.3 & 0.2 & 0.2 & 0.2 & 0.2 & 0.3 & 0.3 & 0.2 & 0.3 & 0.3 \\
\hline $\mathrm{H}_{2} \mathrm{O}$ & 2.2 & 0.4 & 4.8 & 3.5 & 0.8 & 4.4 & 4.8 & 3.6 & 2.8 & 3.3 & 1.1 & 0.9 & 0.5 & 0.6 \\
\hline $\mathrm{S}$ & 0.1 & 0.1 & 0.3 & 0.1 & 0.1 & 0.2 & 0.2 & 0.1 & 0.1 & 0.1 & 0.0 & 0.0 & 0.0 & 0.0 \\
\hline $\mathrm{Cl}$ & 0.1 & 0.1 & 0.1 & 0.1 & 0.1 & 0.1 & 0.1 & 0.1 & 0.1 & 0.1 & 0.1 & 0.1 & 0.1 & 0.1 \\
\hline$P_{\text {cryst-melt }}, \mathrm{MPa}^{\mathrm{d}}$ & 230 & 0.1 & 0.1 & 180 & 680 & 380 & 100 & 370 & 150 & 210 & 130 & 0.1 & 0.1 & 150 \\
\hline$T_{\text {cryst-melt }},{ }^{\circ} \mathrm{C}^{\mathrm{d}}$ & 1014 & 1027 & 981 & 1000 & 1093 & 976 & 936 & 999 & 982 & 980 & 1036 & 967 & 983 & 1034 \\
\hline$T_{\mathrm{S} \& \mathrm{G} 93},{ }^{\circ} \mathrm{C}^{\mathrm{d}}$ & 1087 & 1127 & 1023 & 1029 & 1123 & 1020 & 991 & 1047 & 1043 & 1023 & 1104 & 1136 & 1106 & 1115 \\
\hline$T_{\text {mean }},{ }^{\circ} \mathrm{C}^{\mathrm{d}}$ & 1051 & 1077 & 1002 & 1015 & 1108 & 998 & 964 & 1023 & 1013 & 1002 & 1070 & 1052 & 1045 & 1075 \\
\hline Host-add $(\%)^{\mathrm{e}}$ & 0 & 0 & 8.9 & 9.8 & 0 & 0 & 0 & 0 & 0 & 0 & 0 & 0 & 0 & 0 \\
\hline
\end{tabular}


that Fe-loss has been minor. Also, inclusions trapped by $\mathrm{Ol}, \mathrm{Cpx}$ and $\mathrm{Pl}$ have similar major element compositions at a given $\mathrm{MgO}$ content (Fig. 4), which suggests that effects of post-entrapment crystallization of the host minerals are minor. Nevertheless, these effects can readily be corrected for using appropriate crystal-melt equilibrium models given robust estimates of trapping temperature. We used the PETROLOG program of Danyushevsky (2001) to correct for post-trapping Ol- and Cpx-crystallization, and the Opx-melt model of Ariskin et al. (1993) to account for any Opx crystallization. In all cases, the calculated corrections involved adding less than $10 \mathrm{wt} . \%$ of host mineral, and in most cases less than 3 wt.\% (Table 7). Fig. 4 shows that the correction procedure improves the coherency of inclusion data from different mineral hosts, plotted on major element variation diagrams of $\mathrm{MgO}$ vs. $\mathrm{CaO} / \mathrm{Al}_{2} \mathrm{O}_{3}$. Note that an equivalent correction for post-trapping crystallization in Plhosted inclusions was not made because of the large uncertainty in existing Pl-melt models (discussed in Danyushevsky et al., 1996). However, since plagioclase is commonly the last phase on the liquidus of $\mathrm{H}_{2} \mathrm{O}$-rich, high- $\mathrm{Al}_{2} \mathrm{O}_{3}$ basaltic magma (e.g., Kersting and Arculus, 1994), postentrapment effects should be low compared to other minerals. This is born out by the fact that Pl-hosted inclusions in our study have very similar compositions to those trapped in $\mathrm{Ol}, \mathrm{Cpx}$ and $\mathrm{Opx}$ (Table 7; Fig. 4). Finally it is worth emphasizing that the recalculation procedure employed can only significantly affect the concentrations and ratios of those elements in the melt which are also compatible in the host mineral, and it represents merely a dilution factor for most incompatible minor, trace and volatile elements.

The corrected compositions of glass inclusions and groundmass glasses are plotted in Fig. 5 along with whole-rock data for volcanic rocks from the Kurile island arc taken from the literature (Bailey et al., 1987a,b; Ikeda, 1998; Ikeda et al., 2000; Takagi et al., 1999). Based on their glass compositions, the erupted melts classify as medium- to low-K tholeiitic basalt to andesite, typical for magmas from the Kurile arc (Fig. 5a,b). The compositions of melts from the different eruptive events show considerable overlap, with the 1853 eruption standing out slightly from the others due to more evolved compositions (higher $\mathrm{SiO}_{2}, \mathrm{~K}_{2} \mathrm{O}$ and lower $\mathrm{FeO}$ ). Variations diagrams with $\mathrm{SiO}_{2}$ as a differentiation index show that inclusions and groundmass glasses follow simple trends of decreasing $\mathrm{FeO}, \mathrm{CaO}$ (Fig. 5c,d) and increasing $\mathrm{Na}_{2} \mathrm{O}$ and $\mathrm{K}_{2} \mathrm{O}$ (not shown), which are similar to the whole-rock variation trends defined by the Kurile Island arc data. These differentiation trends can be successfully modelled as liquid lines of descent. Least-squares mixing calculations (not shown) reproduce the range of melt inclusion compositions displayed by any one eruption event by removal of the common phenocryst phases in the proportions: $0-3$ wt.\% of olivine, $7-9$ wt.\% of clinopyroxene, 0-6 wt.\% of orthopyroxene, 10-17 wt. $\%$ of plagioclase and $4-5$ wt. $\%$ of magnetite

\footnotetext{
Notes to Table 7:

${ }^{a}$ Concentrations of major elements in glass inclusions analyzed by electron microprobe; $\mathrm{FeO}_{\text {tot }}=$ total iron as $\mathrm{Fe}^{2+}, \mathrm{H}_{2} \mathrm{O}_{\text {calc }}=$ water contents calculated "by difference" from 100 of the sum of major elements, $\mathrm{S}$ and $\mathrm{Cl}$.

${ }^{\mathrm{b}}$ Composition of host mineral; $\mathrm{Ol}=$ olivine, $\mathrm{Opx}=$ orthopyroxene, $\mathrm{Cpx}=$ clinopyroxene, $\mathrm{Pl}=$ plagioclase; $\mathrm{mg} \#=\mathrm{mol} \% \mathrm{Fo}$ for $\mathrm{Ol}$ and $100 \times \mathrm{Mg} /$ $\left(\mathrm{Mg}+\mathrm{Fe}_{\mathrm{tot}}\right)$, atomic ratio for Opx and $\mathrm{Cpx} ; \mathrm{An}=\mathrm{mol} \%$ anorthite for Pl. $\mathrm{K}_{\mathrm{D}}=$ distribution coefficient between glass inclusion and host. For Ol, Opx and $\mathrm{Cpx} \mathrm{K}_{\mathrm{D}}=(\mathrm{Fe} / \mathrm{Mg})_{\text {cryst }} /(\mathrm{Fe} / \mathrm{Mg})_{\text {melt }}$ calculated using the contents of $\mathrm{FeO}_{\text {tot }}$ in both coexisting phases; for plagioclase, $\mathrm{K}_{\mathrm{D}=}(\mathrm{Ca} /$ $\mathrm{Na})_{\text {plag }} /(\mathrm{Ca} / \mathrm{Na})_{\text {melt }} . \mathrm{ND}=$ not determined, $\mathrm{BDL}=$ below detection limit, $-=$ no value.

${ }^{c}$ Composition of glass inclusions in O1, Cpx and Opx are corrected for post-entrapment crystallization of host mineral; compositions of glass inclusions in $\mathrm{Pl}$ and groundmass are not corrected (see text) but normalized to $100 \%$ after calculation of $\mathrm{Fe}_{2} \mathrm{O}_{3}$ and $\mathrm{FeO}$ contents in the melt, based on the $\mathrm{Fe}^{2+} / \mathrm{Fe}^{3+}$ ratios in inclusions of magnetite coexisting with melt using experimental data of Maurel and Maurel (1982) and the model of Borisov and Shapkin (1990).

${ }^{\mathrm{d}} T_{\text {cryst-melt }}$ given in ${ }^{\circ} \mathrm{C}$ and $P_{\text {cryst-melt }}$ given in MPa-temperature and pressure calculated from the Ol-melt geothermometer of Ford et al. (1983), Cpx-melt and Pl-melt geothermometers of Danyushevsky (2001) and Opx-melt geothermometer of Ariskin et al. (1993) assuming $\mathrm{H}_{2} \mathrm{O}-$ saturated conditions for inclusions in $\mathrm{Ol}, \mathrm{Cpx}$ and $\mathrm{Pl}$ (Danyushevsky, 2001), and dry conditions for Opx; $T_{\mathrm{S} \& \mathrm{G} 93},{ }^{\circ} \mathrm{C}$ - temperature calculated using empirical equation from Sisson and Grove (1993b). $T_{\text {mean }},{ }^{\circ} \mathrm{C}=$ average value for $T_{\text {cryst-melt }}$ and $T_{\mathrm{S}}$ \& G93 presented in Fig. 8 .

${ }^{\mathrm{e}}$ Host-add=amounts of olivine or orthopyroxene given in wt.\% added to melt in order to correct the inclusion compositions for postentrapment crystallization (see text).
} 
(a) The 1986 eruption
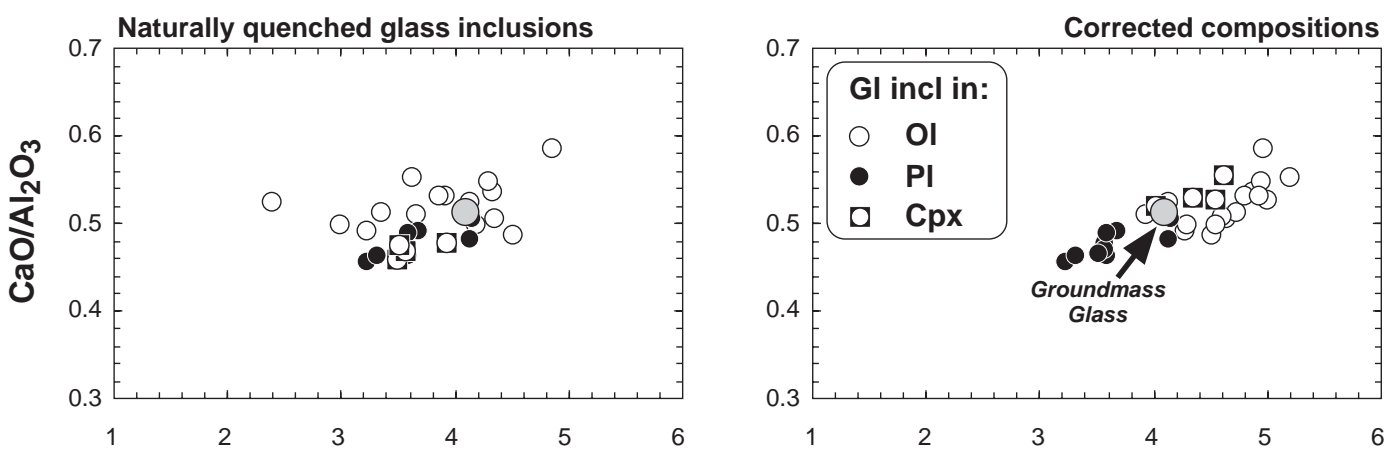

(b) The 1853 eruption
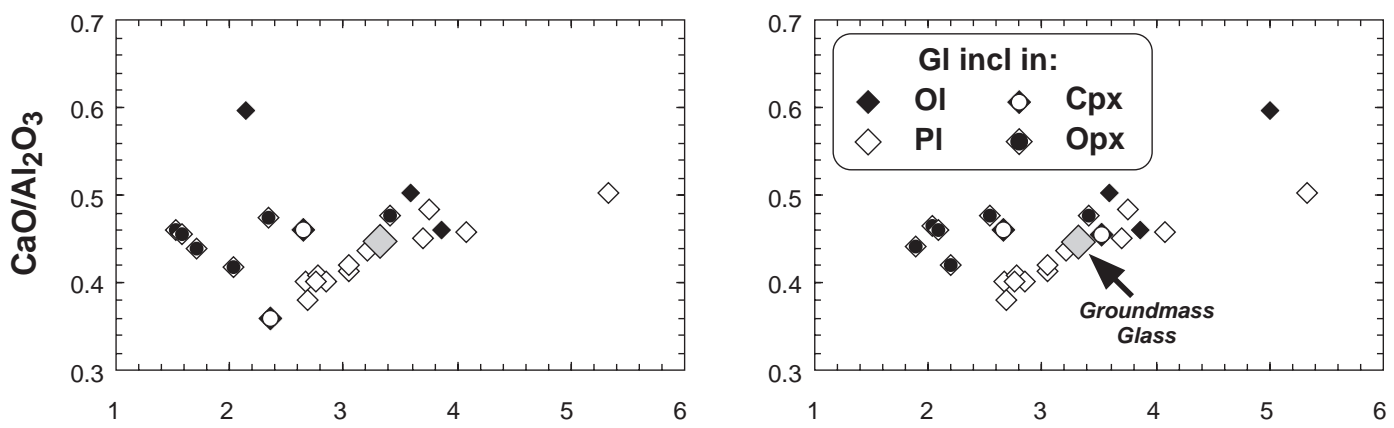

(c) Prehistoric eruption
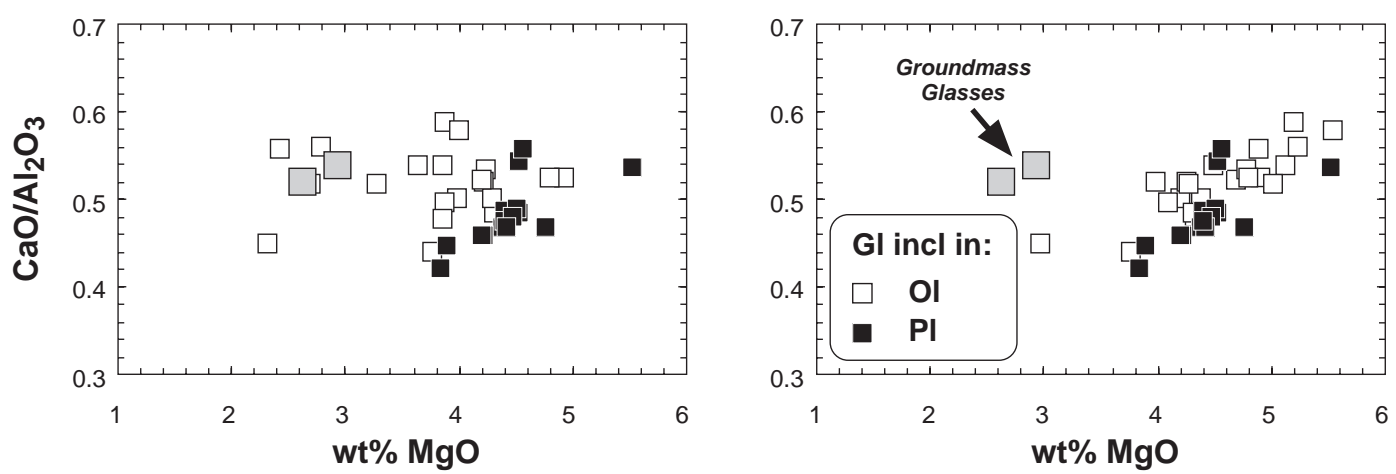

Fig. 4. $\mathrm{CaO} / \mathrm{Al}_{2} \mathrm{O}_{3}$ ratios vs. $\mathrm{MgO}$ contents of glass inclusions in coexisting Ol, $\mathrm{Pl}, \mathrm{Cpx}$ and Opx phenocrysts. The left panels show uncorrected compositions, the right panels present analyses corrected for post-entrapment crystallization of Ol, Cpx and Opx (see text).

(using mineral compositions from Tables 4 and 5, sum of squared residuals $=0.05-0.06$ ).

\subsection{Volatile concentrations}

The concentrations of $\mathrm{H}_{2} \mathrm{O}, \mathrm{S}$ and $\mathrm{Cl}$ in glass inclusions and groundmass glasses are listed in Tables
7 and 8 and selected features are shown in Fig. 6. The results show no systematic differences in volatile contents for the separate eruption events. The volatile concentrations in groundmass glass, which may be taken as a measure for the degassed melt, are 0.31.1 wt. $\% \mathrm{H}_{2} \mathrm{O},<350$ ppm S and 750-930 ppm Cl. The glassy inclusions have a wide range of volatile 

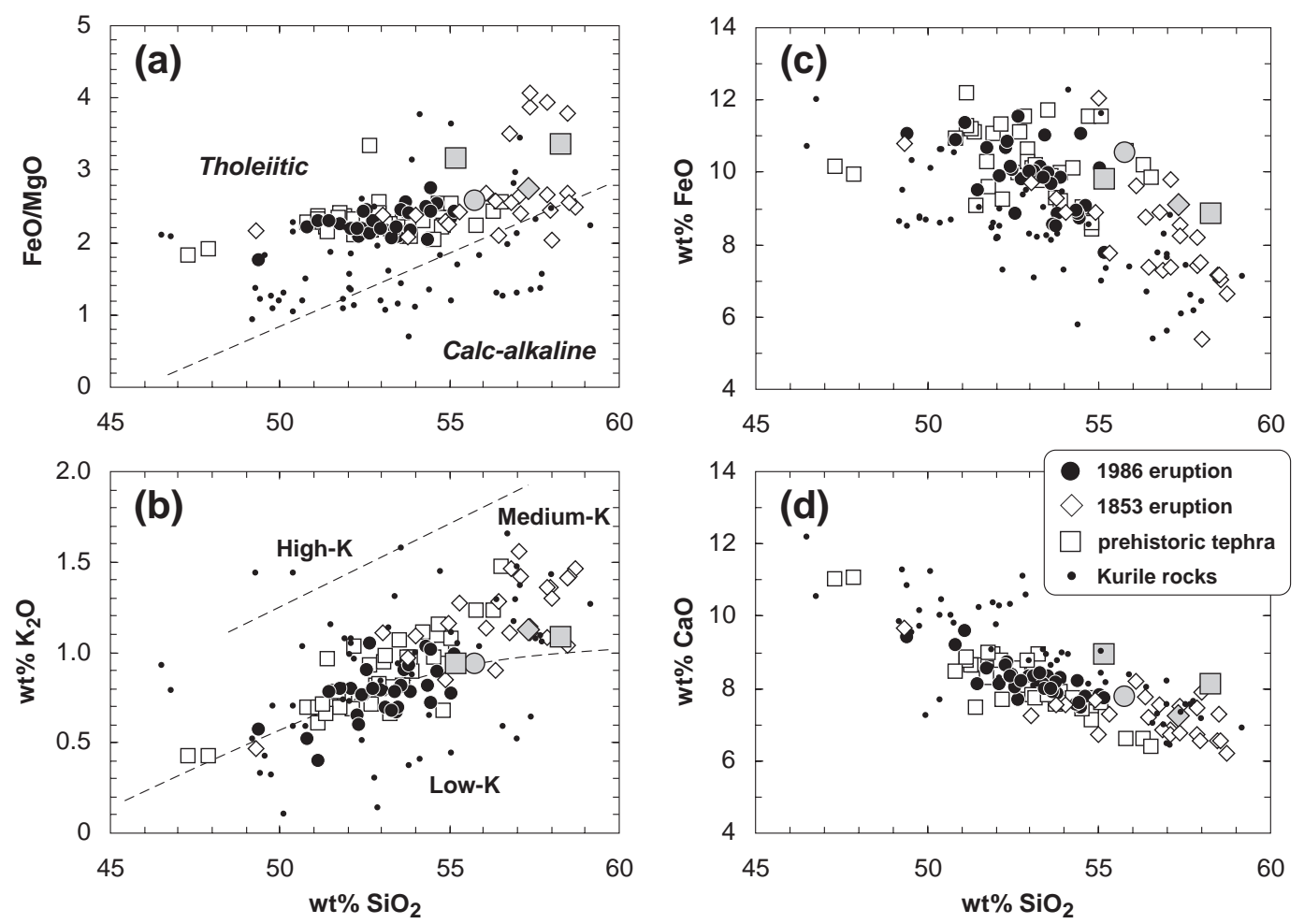

Fig. 5. Major element variations in glass inclusions and groundmass glasses; (a) $\mathrm{FeO} / \mathrm{MgO}$, (b) $\mathrm{K}_{2} \mathrm{O}$, (c) $\mathrm{FeO}$ and (d) $\mathrm{CaO}$ vs. $\mathrm{SiO}$. Field boundaries are from Miyashiro (1974), Tatsumi and Eggins (1995), Arculus (2003). Kurile rocks in the legend are whole-rock data for basalts to andesites (MgO> 2.5 wt.\%) from Bailey et al. (1987a,b), Ikeda (1998), Ikeda et al. (2000) and Takagi et al. (1999). The compositions of glass inclusions, except those trapped in Pl, were corrected for post-entrapment crystallization of host mineral (see Table 7). In this figure and Figs. 6 and 9, small symbols in the legend represent melt inclusions; large shaded symbols represent groundmass glass compositions.

concentrations $\left(0-6.4\right.$ wt.\% $\mathrm{H}_{2} \mathrm{O}, 390-2920$ ppm S, $760-1360 \mathrm{ppm} \mathrm{Cl}$ ) which overlaps at its low end with the groundmass glass, and extends to high values that, especially for $\mathrm{H}_{2} \mathrm{O}$, match those estimated for undegassed magmas from other subduction zone settings (e.g., Sisson and Grove, 1993a,b; Danyushevsky et al., 1993; Sobolev and Chaussidon, 1996; Khubunaya and Sobolev, 1998; Straub and Layne, 2003). There is no correlation between the concentration of $\mathrm{H}_{2} \mathrm{O}$ in the inclusion glasses and incompatible major elements such as $\mathrm{K}_{2} \mathrm{O}$ (Fig. 6a) or $\mathrm{TiO}_{2}$, and $\mathrm{P}_{2} \mathrm{O}_{5}$ (not shown), which suggests that the range in water contents in the trapped melts is not controlled by fractional crystallization but instead is due to variable degrees of degassing in the ambient magma at the time and place of trapping (see discussion). Chlorine also shows no correlation with $\mathrm{K}_{2} \mathrm{O}$ (Fig. 6b), and there appears to be little or no systematic relationships of the volatile components with each other, as indicated by the scatter of $\mathrm{H}_{2} \mathrm{O} / \mathrm{Cl}$ (Fig. 6c) and $\mathrm{H}_{2} \mathrm{O} / \mathrm{S}$ ratios (not shown).

The highest $\mathrm{H}_{2} \mathrm{O}$ contents were found in melt inclusions hosted by plagioclase, with up to 6.4 wt. $\% \mathrm{H}_{2} \mathrm{O}$. It is possible to test whether this value represents the melt composition present at the time of trapping by comparing the $\mathrm{Ca} / \mathrm{Na}$ ratios of plagioclase and melt inclusions with experimentally-determined partitioning of $\mathrm{Ca}$ and $\mathrm{Na}$ between $\mathrm{Pl}$ and coexisting melt as a function of water content. As determined by Sisson and Grove (1993a), the equilibrium constant for $\mathrm{Ca}-\mathrm{Na}$ exchange between plagioclase and melt $\left[\mathrm{K}_{\mathrm{D}}^{\mathrm{Ca}-\mathrm{Na}}=(\mathrm{Ca} / \mathrm{Na})_{\text {plag }} /(\mathrm{Ca} / \mathrm{Na})_{\text {melt }}\right]$ increases from approximately 1.7 for melts with 2 wt. $\% \mathrm{H}_{2} \mathrm{O}$ to 5.5 for melts with 6 wt. $\% \mathrm{H}_{2} \mathrm{O}$ in the pressure range between 100 and $500 \mathrm{MPa}$. Our plagioclase and glass inclusion compositions yield an 
Table 8

Water and trace element concentrations in Ol-hosted glass inclusions analyzed by SIMS

\begin{tabular}{|c|c|c|c|c|c|c|c|c|c|c|c|c|c|c|c|c|c|c|c|c|c|}
\hline \multirow{2}{*}{$\frac{\text { Sample }}{\text { Grain }}$} & \multicolumn{7}{|c|}{ CHK3/4 } & \multicolumn{10}{|c|}{ CHK13-1 } & \multicolumn{2}{|c|}{ CHK13/2 } & \multicolumn{2}{|l|}{ CHK16 } \\
\hline & $1-1$ & $2-1$ & $4-1$ & $9-1$ & $13-1$ & $18-1$ & $\begin{array}{l}3 / \\
4 \mathrm{GL}\end{array}$ & $1-1$ & $1-2$ & $2-1$ & $4-1$ & $9-1$ & $10-1$ & $11-1$ & $19-1$ & $19-2$ & $\begin{array}{l}13 / \\
1 \mathrm{GL}\end{array}$ & $10-1$ & $\begin{array}{l}13 / \\
2 \mathrm{GL}\end{array}$ & $15-1$ & $16 \mathrm{GL}$ \\
\hline$N$ & 1 & - & 2 & 1 & - & - & - & 2 & 1 & 2 & 1 & 2 & 2 & 2 & 1 & 1 & 2 & - & - & - & - \\
\hline $\mathrm{H}_{2} \mathrm{O}$ & 3.42 & ND & 3.27 & 2.57 & ND & ND & ND & 1.47 & 3.61 & 3.28 & 3.1 & 3.30 & 3.40 & 0.94 & 3.40 & 3.50 & 0.27 & ND & ND & ND & ND \\
\hline $1 \sigma$ & 0.02 & - & 0.05 & 0.01 & - & - & - & 0.09 & 0.11 & 0.02 & 0.10 & 0.12 & 0.03 & 0.02 & 0.05 & 0.09 & 0.11 & - & - & - & - \\
\hline$N$ & 1 & 1 & - & 1 & - & 1 & - & 1 & - & 1 & - & 1 & 1 & 1 & - & - & - & 1 & - & - & - \\
\hline $\mathrm{Li}$ & 5.8 & 6.4 & ND & 5.5 & ND & 6.6 & ND & 8.3 & ND & 8.0 & ND & 7.6 & 7.5 & 9.2 & ND & ND & ND & 7.6 & ND & ND & ND \\
\hline $1 \sigma$ & 0.1 & 0.1 & - & 0.1 & - & 0.1 & - & 0.1 & - & 0.1 & - & 0.1 & 0.1 & 0.1 & - & - & - & 0.1 & - & - & - \\
\hline $\mathrm{Be}$ & 0.41 & 0.49 & ND & 0.43 & ND & 0.41 & ND & 0.58 & ND & 0.53 & ND & 0.55 & 0.53 & 0.57 & ND & ND & ND & 0.52 & ND & ND & ND \\
\hline $1 \sigma$ & 0.01 & 0.02 & - & 0.01 & - & 0.01 & - & 0.02 & - & 0.02 & - & 0.02 & 0.02 & 0.02 & - & - & - & 0.02 & - & - & - \\
\hline B & 16.9 & 17.6 & ND & 17.2 & ND & 16.1 & ND & 18.5 & ND & 17.0 & ND & 17.4 & 16.9 & 18.7 & ND & ND & ND & 18.1 & ND & ND & ND \\
\hline $1 \sigma$ & 0.1 & 0.2 & - & 0.2 & - & 0.1 & - & 0.2 & - & 0.2 & - & 0.2 & 0.2 & 0.2 & - & - & - & 0.2 & - & - & - \\
\hline$N$ & 1 & 1 & - & - & 1 & 1 & 2 & 2 & 1 & 1 & 1 & 1 & 1 & 1 & 1 & 1 & 2 & 1 & 1 & 1 & 2 \\
\hline K & 6656 & 7788 & ND & ND & 8095 & 6090 & 7380 & 6034 & 6020 & 6342 & 7617 & 5566 & 5545 & 6897 & 7612 & 7032 & 7370 & 6436 & 8304 & 11,055 & 8761 \\
\hline $\mathrm{Ti}$ & 6756 & 6977 & ND & ND & 3968 & 5481 & 6810 & 5816 & 5834 & 5802 & 8320 & 5642 & 5501 & 6344 & 5810 & 6209 & 8434 & 5602 & 6594 & 4254 & 5309 \\
\hline V & 476 & 408 & ND & ND & 226 & 369 & 400 & 384 & 411 & 423 & 676 & 369 & 375 & 431 & 314 & 360 & 687 & 356 & 339 & 213 & 247 \\
\hline $\mathrm{Cr}$ & 22.0 & 21.1 & ND & ND & 10.4 & 15.9 & 18.4 & 16.7 & 16.7 & 18.4 & 22.7 & 16.0 & 18.5 & 17.9 & 81.6 & 13.4 & 15.2 & 14.4 & 10.6 & 9.5 & 9.7 \\
\hline $\mathrm{Sr}$ & 402 & 467 & ND & ND & 447 & 443 & 389 & 448 & 425 & 423 & 386 & 448 & 417 & 459 & 369 & 385 & 407 & 430 & 418 & 382 & 449 \\
\hline $\mathrm{Ba}$ & 214 & 215 & ND & ND & 237 & 199 & 227 & 207 & 194 & 206 & 229 & 204 & 200 & 225 & 210 & 206 & 222 & 215 & 246 & 278 & 252 \\
\hline Y & 25.8 & 31.2 & ND & ND & 19.8 & 25.0 & 30.8 & 23.7 & 22.6 & 22.7 & 26.0 & 24.3 & 22.6 & 24.4 & 23.0 & 26.3 & 26.1 & 24.5 & 28.9 & 22.1 & 26.3 \\
\hline $\mathrm{Zr}$ & 64.5 & 70.8 & ND & ND & 57.2 & 55.2 & 74.8 & 55.8 & 56.7 & 54.3 & 67.6 & 55.6 & 52.9 & 62.6 & 63.3 & 69.5 & 65.5 & 58.5 & 72.3 & 72.3 & 71.7 \\
\hline $\mathrm{Nb}$ & 1.40 & 1.96 & ND & ND & 1.27 & 1.40 & 1.53 & 1.28 & 1.34 & 1.35 & 1.44 & 1.32 & 1.19 & 1.32 & 1.39 & 1.42 & 1.41 & 1.40 & 1.92 & 1.60 & 1.70 \\
\hline Hf & 2.05 & 0.71 & ND & ND & 2.03 & 2.74 & 2.01 & 2.66 & 0.56 & 3.11 & 2.41 & 1.78 & 1.34 & 2.01 & 1.05 & 3.23 & 0.49 & 1.02 & 2.51 & 2.52 & 1.62 \\
\hline Th & 1.33 & 1.11 & ND & ND & 1.93 & 1.05 & 1.55 & 1.31 & 1.04 & 1.05 & 1.08 & 1.05 & 1.19 & 1.13 & 1.25 & 1.27 & 1.02 & 1.09 & 1.22 & 2.47 & 2.00 \\
\hline $\mathrm{La}$ & 6.83 & 7.23 & ND & ND & 7.0 & 6.11 & 7.27 & 6.92 & 6.35 & 6.23 & 7.10 & 6.81 & 6.55 & 7.28 & 6.6 & 6.7 & 7.17 & 6.92 & 7.51 & 7.66 & 7.38 \\
\hline $\mathrm{Ce}$ & 17.5 & 17.2 & ND & ND & 16.73 & 15.5 & 18.9 & 16.5 & 15.5 & 15.4 & 18.1 & 16.5 & 15.8 & 17.5 & 17.42 & 18.33 & 17.3 & 15.7 & 19.1 & 17.9 & 18.7 \\
\hline $\operatorname{Pr}$ & 2.50 & 2.37 & ND & ND & 2.7 & 2.59 & 3.09 & 2.52 & 2.54 & 2.52 & 2.42 & 2.31 & 2.47 & 2.71 & 2.3 & 2.8 & 2.48 & 2.49 & 2.76 & 2.54 & 2.85 \\
\hline $\mathrm{Nd}$ & 12.2 & 12.9 & ND & ND & 10.93 & 11.9 & 14.04 & 11.3 & 11.0 & 10.9 & 11.8 & 12.5 & 11.3 & 12.2 & 10.19 & 11.62 & 12.0 & 12.1 & 12.8 & 11.4 & 13.3 \\
\hline $\mathrm{Sm}$ & 3.70 & 3.65 & ND & ND & 3.03 & 3.07 & 4.27 & 3.43 & 3.10 & 3.35 & 3.84 & 2.95 & 2.90 & 3.46 & 2.95 & 3.24 & 3.38 & 3.39 & 3.88 & 3.4 & 2.97 \\
\hline $\mathrm{Eu}$ & 1.32 & 1.09 & ND & ND & 0.90 & 0.91 & 1.43 & 1.15 & 0.90 & 1.17 & 1.12 & 1.21 & 1.15 & 1.22 & 0.77 & 1.21 & 1.15 & 1.01 & 1.36 & 0.98 & 0.93 \\
\hline $\mathrm{Gd}$ & 4.15 & 4.85 & ND & ND & 4.33 & 3.82 & 5.21 & 4.49 & 2.85 & 3.97 & 5.32 & 3.78 & 3.89 & 3.57 & 3.95 & 4.30 & 3.68 & 4.58 & 4.24 & 3.26 & 4.06 \\
\hline $\mathrm{Tb}$ & 0.70 & 0.68 & ND & ND & 0.94 & 0.78 & 0.78 & 0.57 & 0.48 & 0.54 & 0.83 & 0.58 & 0.49 & 0.54 & 0.60 & 0.52 & 0.53 & 0.79 & 0.62 & 0.52 & 0.78 \\
\hline Dy & 4.44 & 4.21 & ND & ND & 3.54 & 4.35 & 4.99 & 4.58 & 4.35 & 3.60 & 5.33 & 3.96 & 3.72 & 4.03 & 4.71 & 4.83 & 5.03 & 3.96 & 4.99 & 4.04 & 4.34 \\
\hline Но & 1.02 & 0.93 & ND & ND & 0.72 & 0.87 & 1.14 & 0.87 & 1.01 & 0.82 & 0.89 & 0.91 & 0.76 & 0.91 & 0.87 & 0.99 & 0.89 & 0.92 & 1.06 & 0.80 & 1.02 \\
\hline $\mathrm{Er}$ & 2.71 & 2.81 & ND & ND & 2.35 & 2.56 & 3.24 & 2.71 & 2.33 & 2.40 & 3.80 & 2.39 & 2.34 & 2.63 & 3.31 & 3.28 & 3.27 & 2.73 & 3.14 & 2.27 & 2.21 \\
\hline $\mathrm{Tm}$ & 0.36 & 0.61 & ND & ND & 0.38 & 0.34 & 0.53 & 0.34 & 0.48 & 0.36 & 0.34 & 0.38 & 0.24 & 0.41 & 0.17 & 0.36 & 0.32 & 0.35 & 0.46 & 0.38 & 0.46 \\
\hline $\mathrm{Yb}$ & 2.98 & 2.80 & ND & ND & 2.25 & 2.89 & 3.53 & 2.46 & 2.69 & 2.59 & 2.88 & 2.66 & 2.31 & 2.90 & 2.57 & 2.91 & 3.22 & 2.75 & 3.65 & 2.33 & 2.80 \\
\hline $\mathrm{Lu}$ & 0.39 & 0.53 & ND & ND & 0.43 & 0.36 & 0.76 & 0.51 & 0.46 & 0.40 & 0.73 & 0.34 & 0.37 & 0.37 & 0.75 & 0.65 & 0.95 & 0.30 & 0.60 & 0.32 & 0.30 \\
\hline$(\mathrm{La} / \mathrm{Sm})_{n}$ & 1.94 & 2.08 & - & - & 2.43 & 2.09 & 1.79 & 2.12 & 2.15 & 1.96 & 1.95 & 2.43 & 2.38 & 2.21 & 2.35 & 2.18 & 2.23 & 2.15 & 2.04 & 2.34 & 2.61 \\
\hline$(\mathrm{Nb} / \mathrm{La})_{n}$ & 0.22 & 0.29 & - & - & 0.19 & 0.25 & 0.23 & 0.20 & 0.23 & 0.23 & 0.22 & 0.21 & 0.19 & 0.19 & 0.23 & 0.23 & 0.21 & 0.22 & 0.27 & 0.22 & 0.25 \\
\hline$(\mathrm{Zr} / \mathrm{Sm})_{n}$ & 0.62 & 0.69 & - & - & 0.67 & 0.64 & 0.62 & 0.58 & 0.65 & 0.58 & 0.63 & 0.67 & 0.65 & 0.64 & 0.76 & 0.76 & 0.69 & 0.61 & 0.66 & 0.75 & 0.86 \\
\hline$(\mathrm{Ti} / \mathrm{Gd})_{n}$ & 0.79 & 0.70 & - & - & 0.44 & 0.69 & 0.63 & 0.63 & 0.99 & 0.71 & 0.76 & 0.72 & 0.68 & 0.86 & 0.71 & 0.70 & 1.11 & 0.59 & 0.75 & 0.63 & 0.63 \\
\hline$(\mathrm{Ba} / \mathrm{Nb})_{n}$ & 56.5 & 40.6 & - & - & 69.0 & 52.6 & 54.9 & 59.8 & 53.5 & 56.4 & 58.8 & 57.2 & 62.2 & 63.0 & 55.9 & 53.7 & 58.2 & 56.8 & 47.4 & 64.3 & 54.8 \\
\hline$(\mathrm{Th} / \mathrm{Nb})_{n}$ & 18.4 & 11.0 & - & - & 29.5 & 14.6 & 19.7 & 19.9 & 15.1 & 15.1 & 14.6 & 15.4 & 19.4 & 16.6 & 17.5 & 17.4 & 14.0 & 15.1 & 12.3 & 30.0 & 22.8 \\
\hline$(\mathrm{K} / \mathrm{Nb})_{n}$ & 18.5 & 15.4 & - & - & 24.8 & 16.9 & 18.7 & 18.3 & 17.4 & 18.2 & 20.5 & 16.4 & 18.1 & 20.3 & 21.3 & 19.2 & 20.3 & 17.9 & 16.8 & 26.8 & 20.0 \\
\hline$(\mathrm{Sr} / \mathrm{Ce})_{n}$ & 1.91 & 2.26 & - & - & 2.23 & 2.38 & 1.72 & 2.26 & 2.28 & 2.29 & 1.78 & 2.26 & 2.20 & 2.19 & 1.77 & 1.75 & 1.96 & 2.28 & 1.82 & 1.78 & 2.00 \\
\hline
\end{tabular}

Glass inclusions were analyzed for $\mathrm{Li}, \mathrm{Be}$ and $\mathrm{B}$ (given in parts per million) using the Cameca ims 6f ion microprobe at GFZ Potsdam, and for $\mathrm{H}_{2} \mathrm{O}$ (given in weight percent) and trace elements (given in parts per million) using the Cameca ims $3 \mathrm{f}$ ion microprobe at MPI for Chemistry (Mainz); $N=$ number of replicates, $1 \sigma$ represents internal analytical precision in case of one measurement (each measurement involves 6 cycles (water) or 5 cycles (traces), thus the internal precision is the $1 \sigma \mathrm{SD}$ of the cycles relative to the mean value) and indicates a deviation from the mean in case of two measurements. Element ratios are given normalized to N-MORB composition (Hofmann, 1988). 

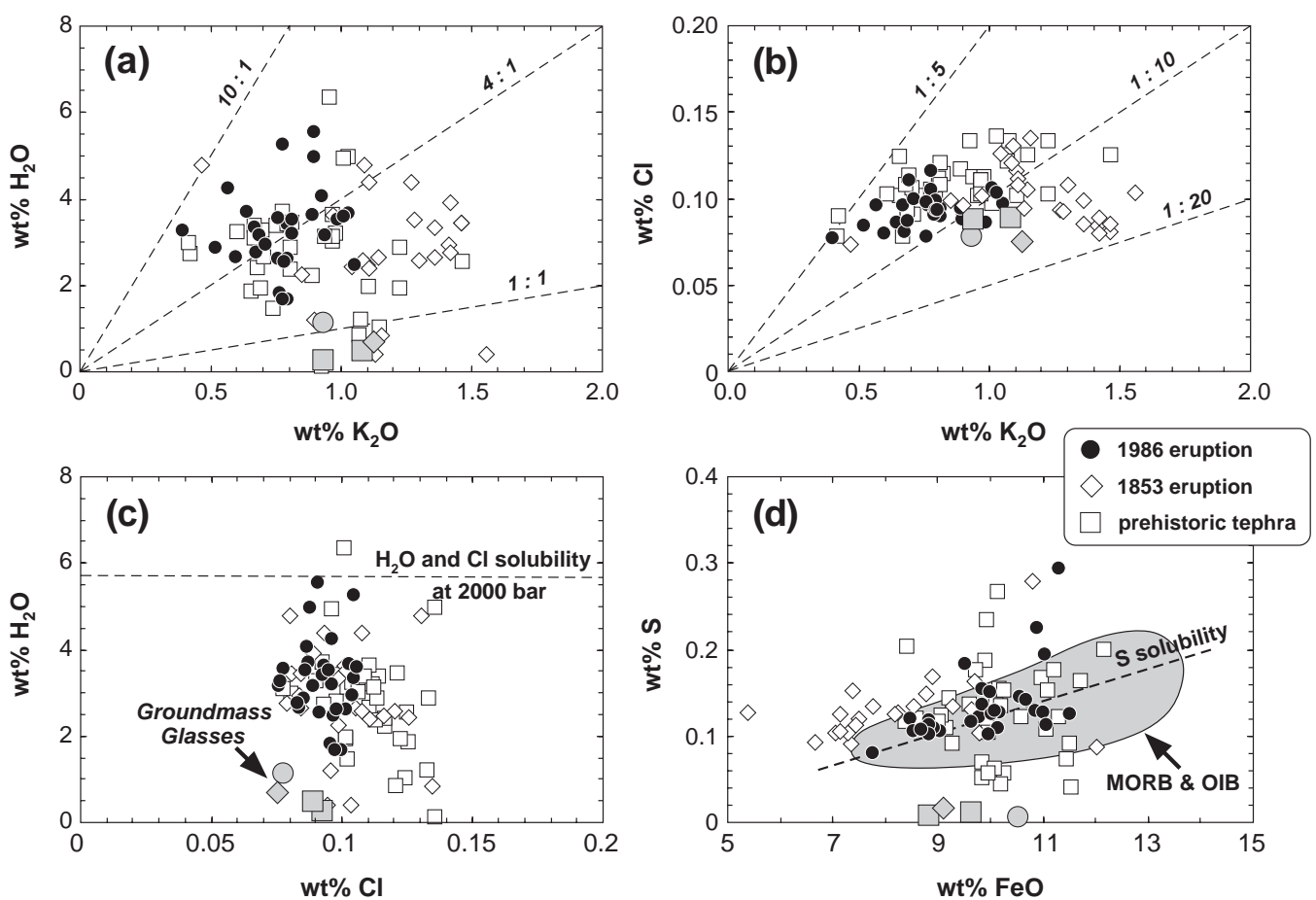

Fig. 6. $\mathrm{H}_{2} \mathrm{O}, \mathrm{S}$ and $\mathrm{Cl}$ concentrations in glass inclusions and glassy matrix. (a) $\mathrm{H}_{2} \mathrm{O}$ vs. $\mathrm{K}_{2} \mathrm{O}$, (b) $\mathrm{Cl}$ vs. $\mathrm{H}_{2} \mathrm{O}$, (c) $\mathrm{H}_{2} \mathrm{O}$ vs. $\mathrm{Cl}$ and (d) $\mathrm{S}$ vs. $\mathrm{FeO}$. The $\mathrm{H}_{2} \mathrm{O}$ data were obtained by SIMS and electron microprobe "by difference" method. The latter are reported only for samples lacking SIMS data. The $\mathrm{H}_{2} \mathrm{O}$ and $\mathrm{Cl}$ solubility isobar for $200 \mathrm{MPa}$ in basaltic melts (panel c) is from Webster et al. (1999). Concentrations of $\mathrm{H}_{2} \mathrm{O}$ and $\mathrm{Cl}$ dissolved in glass inclusions suggest minimum pressure of phenocryst crystallization and melt inclusion trapping corresponding to $200 \mathrm{MPa}$. The MORB and OIB shaded field (panel d) contains data of Wallace and Carmichael (1992 and references therein). Solid line represents saturation of basaltic magmas with sulfide sulfur (Mathez, 1976).

overall mean value for $\mathrm{K}_{\mathrm{D}}^{\mathrm{Ca}-\mathrm{Na}}$ of $4.5 \pm 0.9$ (Table 7), which agrees well with the experimental $\mathrm{K}_{\mathrm{D}}$-values for relatively $\mathrm{H}_{2} \mathrm{O}$-rich melts. This indicates that relatively high $\mathrm{H}_{2} \mathrm{O}$ contents of Pl-hosted inclusions were not a result of post-entrapment crystallization but represent real $\mathrm{H}_{2} \mathrm{O}$ contents in the magma plumbing system.

Our data for $\mathrm{S}$ and $\mathrm{FeO}$ in Chikurachki glasses are plotted together with literature data for MORB and OIB magmas for comparison on Fig. 6d. Sulfur solubility in basaltic magmas has been shown to be a function of iron content if $\mathrm{S}$ is dissolved as sulfide, and the corresponding solubility limit from Mathez (1976) is also shown on Fig. 6d. Many of the Chirurachki glass inclusions have higher $\mathrm{S}$ concentrations than the MORB or OIB field, whereas the groundmass glasses are consistently much lower in $\mathrm{S}(<350 \mathrm{ppm})$, consistent with loss of $\mathrm{S}$ by eruption degassing. There is no correlation of $\mathrm{S}$ with $\mathrm{FeO}$ in the inclusion glasses as would be expected for S dissolved mostly as sulfide, and also, many of the measured $\mathrm{S}$ concentrations exceed the solubility curve of Mathez (1976). We interpret this to indicate a higher proportion of sulfur is dissolved as sulfate than in MORB or OIB magmas, which is consistent with the higher oxidation state of subduction-zone magmas. Estimated oxygen fugacities in the Chikurachki magmas are $\Delta \mathrm{NNO}+1$ to +2 (see below), and in accordance with data of Wallace and Carmichael (1994 and references therein), around $60 \%$ to $80 \%$ of sulfur dissolved in the melt under these conditions could be present as $\mathrm{SO}_{4}^{2-}$. Like $\mathrm{H}_{2} \mathrm{O}$, the range of $\mathrm{S}$ values in melt inclusions is large, and we interpret inclusions with lower $\mathrm{S}$ concentrations than the MORB or OIB field to have partially lost S by degassing.

Measured chlorine concentrations in melt inclusions range from about 0.08 to 0.13 wt.\% (Fig. 6), and concentrations in the groundmass glass are at the 
low end of this range (0.07-0.09 wt.\%). The poor correlations of $\mathrm{Cl}$ with $\mathrm{H}_{2} \mathrm{O}$ and $\mathrm{S}$ in the Chikurachki melt inclusions, and the low to negligible differences in $\mathrm{Cl}$ concentrations between melt inclusions and groundmass glasses, indicates that degassing of $\mathrm{Cl}$ appears to have been much less effective in these magmas than water or sulfur. The solubility of $\mathrm{Cl}$ in silicate melts is a complex function of $\mathrm{P}-\mathrm{T}$ and melt composition (e.g., Metrich and Rutherford, 1992; Webster, 1992; Carroll and Webster, 1994; Webster et al., 1999; Signorelli and Carroll, 2001). Whereas chlorine solubility in hydrous, alkaline-poor, siliceous melts is low, leading to the potential for $\mathrm{Cl}$ oversaturation and loss during crystallization, basaltic and andesitic magmas may remain undersaturated in $\mathrm{Cl}$, at least until water saturation is achieved (Webster et al., 1999; Signorelli and Carroll, 2001). However, as shown by Stix and Layne (1996), the distribution coefficient for $\mathrm{Cl}$ between melt and aqueous vapor is moderate, so even after water saturation there will not be a strong depletion of $\mathrm{Cl}$ from the melt.

\subsection{Trace elements}

Trace elements in melt inclusions and groundmass glasses are listed in Table 8 and selected features are presented in Fig. 7. There are no significant differences in trace element concentrations between glass inclusions and groundmass glasses, nor do the separate eruption events from Chikurachki stand out in the trace element plots. Overall, the glass inclusions are slightly enriched in light rare earth elements (LREE) relative to the heavy rare-earths (HREE); e.g. $[\mathrm{La} / \mathrm{Sm}]_{n}=1.8-2.6$, where the subscript $n$ means normalized to N-MORB composition from Hofmann (1988). They also show significant depletions in high-field-strength elements (HFSE) relative to N-MORB (e.g., $[\mathrm{Nb} /$ $\mathrm{La}]_{n}=0.2-0.3, \quad[\mathrm{Zr} / \mathrm{Sm}]_{n}=0.6-0.9, \quad[\mathrm{Ti} / \mathrm{Gd}]_{n}=0.4-$ 1.1) and enrichments in Th and the large-ion lithophile elements (LILE) (e.g., $[\mathrm{Ba} / \mathrm{Nb}]_{n}=41-69$, [Th/ $\mathrm{Nb}]_{n}=11-30,[\mathrm{~K} / \mathrm{Nb}]_{n}=15-27$ and $[\mathrm{Sr} / \mathrm{Ce}]_{n}=1.7-$ 2.4) (Fig. 7). The high field-strength elements $\mathrm{Y}, \mathrm{Zr}$, $\mathrm{Nb}$ and $\mathrm{Yb}$ show good positive correlations with each other $(\mathrm{R}>0.7)$. These compositional features are typical for subduction zone-related magmas and are widely attributed to the selective addition of elements released from the subducted slab and introduced by melt and/or fluid transport into the depleted mantle wedge (e.g., Arculus, 1994; Pearce and Peate, 1995; Tatsumi and Eggins, 1995).

Concentrations of fluid-mobile trace elements $\mathrm{Li}$ (5.8-9.2 ppm), Be (0.41-0.58 ppm) and B (16.1$18.7 \mathrm{ppm}$ ) in the glasses (Table 8) are in the mid range of concentrations reported for Kurile arc magmas with $\mathrm{SiO}_{2}<60$ wt.\% (1.0-17 ppm Li, 0.19$1.26 \mathrm{ppm}$ Be and 4.5-36.6 ppm B; Bailey et al., 1987a,b; Ryan et al., 1995; Ishikawa and Tera,

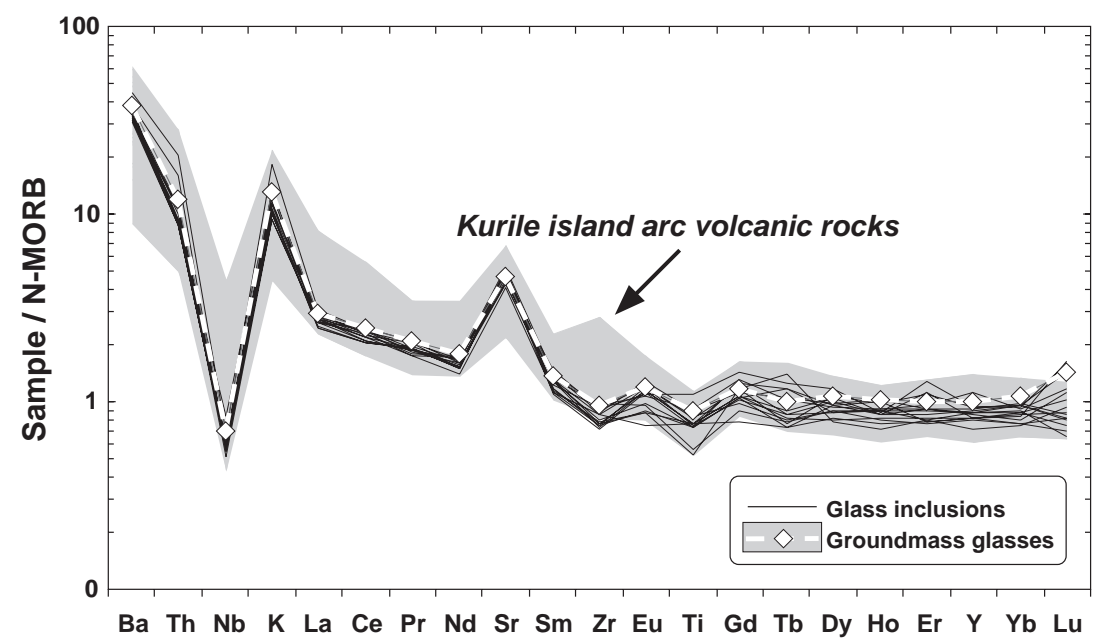

Fig. 7. Trace element distribution in glass inclusions normalized to N-MORB composition (Hofmann, 1988), and shown in comparison to whole-rock data (shaded field) of the Kurile Arc basalts to andesites (data sources as in Fig. 5). 
1997). Ishikawa and Tera (1997) found very similar concentrations of B in the Chikurachki lavas (14.4$15.5 \mathrm{ppm})$ to our results. In general, the concentrations of $\mathrm{Be}, \mathrm{B}$ and $\mathrm{Li}$ show good positive correlations with each other and with $\mathrm{Cl}$, and this group also tends to show positive correlations with other potentially fluid-mobile elements $\mathrm{Ba}, \mathrm{Sr}$ and even La (correlation coefficients $R>0.7$ ). These correlations are greatly improved if, instead of element concentrations, ratios to a fluid-immobile incompatible element (e.g., $\mathrm{Nb}$ in Fig. 8) are used to minimize effects of variable partial melting or fractionation. In the geologic setting of island arc magmatism relevant to Chikurachki, it is reasonable to assume that the main source of $\mathrm{Cl}$ and other fluid-mobile incompatible elements $(\mathrm{Li}, \mathrm{Be}, \mathrm{B}, \mathrm{Ba}$, $\mathrm{Sr}$ ) is ultimately the subducting slab and not the mantle wedge (e.g., Brenan et al., 1995; Ayers et al., 1997; Stalder et al., 1998). The strong positive correlations of $\mathrm{Cl} / \mathrm{Nb}$ with $(\mathrm{Li}, \mathrm{Be}, \mathrm{B}, \mathrm{Ba}, \mathrm{Sr}) / \mathrm{Nb}$ ratios in the inclusion glasses suggests that these elements have a common source (slab component) and that their distribution in the erupted magmas is controlled by transport by a Cl-rich fluid.

\section{Intensive parameters of magma crystallization}

\subsection{Temperature}

The temperature range of magma crystallization was estimated using (1) mineral geothermometry based on equilibria among Ol, Opx, Cpx and Fe-Ti oxides, with calibrations incorporated in the QUILF program (Andersen et al., 1993); (2) mineral-melt geothermometers using compositions of melt inclusions and host crystals, using expressions of Ford et al. (1983) for inclusions in Ol, and Danyushevsky (2001) for inclusions in Pl and Cpx, realized in the PETROLOG program (Danyushevsky, 2001); and (3)
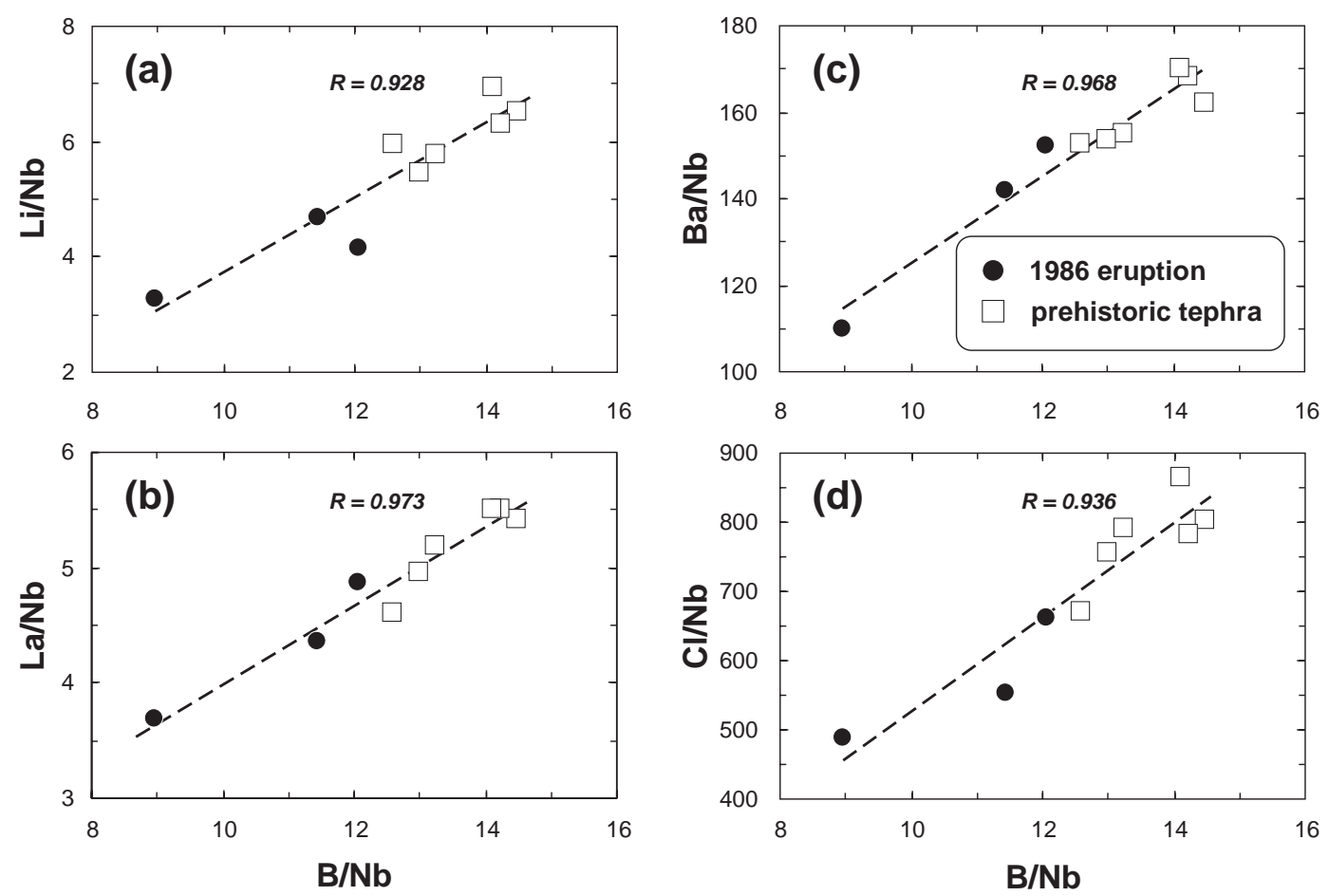

Fig. 8. Variation diagram of selected fluid-mobile trace elements and La normalized to $\mathrm{Nb}$ and plotted against $\mathrm{B} / \mathrm{Nb}$ ratio. (a) $\mathrm{Li} / \mathrm{Nb}$, (b) $\mathrm{La} / \mathrm{Nb}$, (c) $\mathrm{Ba} / \mathrm{Nb}$ and (d) $\mathrm{Cl} / \mathrm{Nb}$ vs. $\mathrm{B} / \mathrm{Nb}$ ratios. Normalization to $\mathrm{Nb}$ minimizes effects of differential partial melting and fractional crystallization on element concentrations, and reveals strong positive correlation for these elements (see text). 
empirical equation of Sisson and Grove (1993b) relating temperature, pressure, liquid composition and melt $\mathrm{H}_{2} \mathrm{O}$ contents (Fig. 9, Table 9).

From experimental data of Sisson and Grove (1993a,b) and Grove et al. (2003), we selected 27 glasses whose compositions are similar to those of

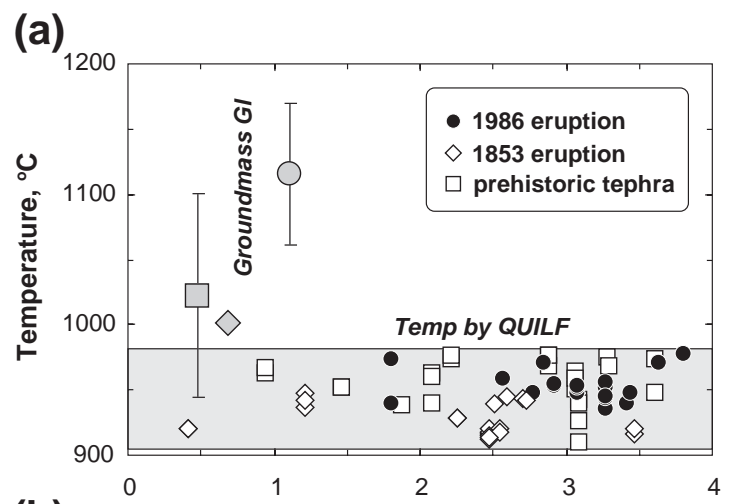

(b)

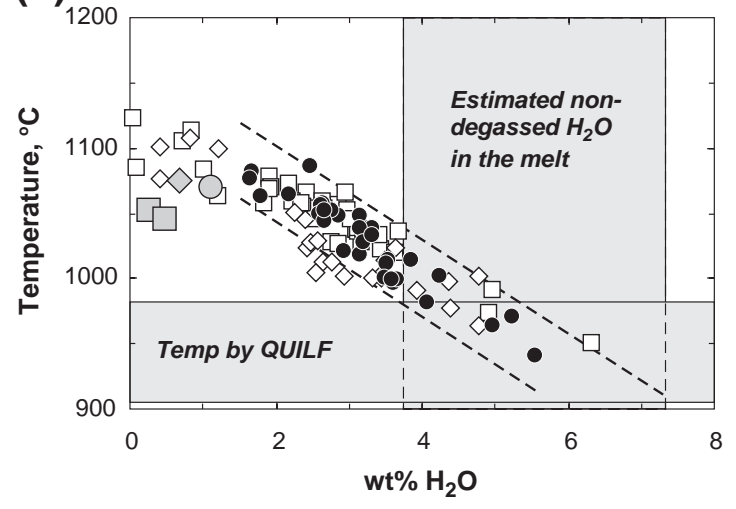

Fig. 9. Temperature of magma crystallization vs. $\mathrm{H}_{2} \mathrm{O}$ contents in the melt. (a) Temperatures calculated using the QUILF program (Andersen et al., 1993) and compositions of Ol, Cpx, Opx phenocrysts, $\mathrm{Sp}$ inclusions and microphenocrysts in groundmass glasses. The $\mathrm{H}_{2} \mathrm{O}$ contents on the $X$-axis are compositions of phenocryst-hosted melt inclusions and groundmass glasses. Error bars on the groundmass glasses represent $\mathrm{a} \pm 1$ sigma standard deviation (Table 9). (b) Temperatures calculated from mineralmelt geothermometers for inclusion glass-host crystal pairs (Danyushevsky, 2001; Sisson and Grove, 1993b). These temperatures show strong inverse correlation with $\mathrm{H}_{2} \mathrm{O}$ contents of glass inclusions, and agree with QUILF results only for the water-rich glasses. The temperatures calculated for water-poor inclusions are thought to be overestimates due to $\mathrm{H}_{2} \mathrm{O}$ degassing and using water-dependent geothermometers (see text). Intersection of the $\mathrm{H}_{2} \mathrm{O}-T$ array from mineral-melt thermometry with the QUILF field gives an estimate of non-degassed water contents in the melt between approximately 4 and 7 wt.\% $\mathrm{H}_{2} \mathrm{O}$ (see text). the Chikurachki inclusions and calculated temperatures using the geothermometers employed in our study. The temperatures calculated from the Sisson and Grove (1993b) equation agreed with the experimental temperatures within $30-40{ }^{\circ} \mathrm{C}$. The plagioclase-melt geothermometer of Danyushevsky (2001) yielded a greater deviation from experimental temperatures (average $70{ }^{\circ} \mathrm{C}$ ), with both over- and underestimation of the experimental temperature, while the two-pyroxene geothermometer (QUILF) systematically overestimated the experimental temperatures by an average of $\sim 60{ }^{\circ} \mathrm{C}$. The test of QUILF geothermometer used in single-pyroxene mode provided an uncertainty of $\pm 15-35^{\circ} \mathrm{C}$ for most glasses, or for some cases, as high as $\pm 70{ }^{\circ} \mathrm{C}$.

It is instructive to compare the temperatures calculated from methods that are independent of melt water contents and those where water is explicitly included. The QUILF temperatures are based on exchange equilibria among coexisting $\mathrm{H}_{2} \mathrm{O}$-free phases $(\mathrm{Ol}, \mathrm{Cpx}$, Pig, Opx, Sp), and the temperatures obtained range from 910 to $980{ }^{\circ} \mathrm{C}$. When individual QUILF temperatures are plotted against $\mathrm{H}_{2} \mathrm{O}$ contents of melt inclusions hosted by the phenocrysts used for calculations (Fig. 9a), there is no relationship between temperature and melt water content. By contrast, temperatures calculated from mineral-melt equilibria of Sisson and Grove (1993b) and Danyushevsky (2001) show a strong and systematic variation with melt water contents (Fig. 9b). Examination of Table 9 shows that the Sisson and Grove (1993a,b) temperatures are systematically higher than those calculated from Danyushevsky (2001), probably because the effect of $\mathrm{H}_{2} \mathrm{O}$ on crystallization temperature is handled differently in these two models. However, the deviation is typically $40-60{ }^{\circ} \mathrm{C}$, which is on the same order of a realistic standard error for the temperature calculations of $\pm 40{ }^{\circ} \mathrm{C}$, and therefore the difference between the methods is insignificant at the $95 \%$ confidence level. We emphasize that the inverse correlation of temperatures with melt $\mathrm{H}_{2} \mathrm{O}$ contents showed by mineral-melt geothermometry (Fig. 9b) does not necessarily reflect real variations in crystallization temperatures. The water-independent QUILF results agree with mineral-melt geothermometers only for inclusions with high melt water contents. For the others, we believe that the mineral-melt temperatures are overestimated because inclusions have 
Table 9

Estimated temperature, pressure and redox conditions of magma crystallization

\begin{tabular}{|c|c|c|c|c|c|c|c|}
\hline \multirow[t]{2}{*}{ Sample } & \multirow[t]{2}{*}{$\begin{array}{l}\text { Geothermo/ } \\
\text { barometer, Ref. }\end{array}$} & \multicolumn{2}{|c|}{ CHK3/4 1986 eruption } & \multicolumn{2}{|c|}{ CHK16 1853 eruption } & \multicolumn{2}{|c|}{$\begin{array}{l}\text { CHK13/1\&CHK13/2 } \\
\text { Prehistoric eruption }\end{array}$} \\
\hline & & Interval & Mean $\pm 1 \sigma$ & Interval & Mean $\pm 1 \sigma$ & Interval & Mean $\pm 1 \sigma$ \\
\hline \multicolumn{8}{|c|}{ Temperature $\left({ }^{\circ} \mathrm{C}\right)^{\mathrm{a}}$} \\
\hline \multirow[t]{2}{*}{$\mathrm{Incl}$ in $\mathrm{Ol}$} & Ol-melt, DN2001 & $989-1037$ & - & $981-1027$ & - & $979-1078$ & - \\
\hline & S\&G93 & $1033-1127$ & - & $1023-1127$ & - & $1042-1168$ & - \\
\hline \multirow[t]{2}{*}{ Incl in $\mathrm{Pl}$} & Pl-melt, DN2001 & $914-1019$ & - & 937-999 & - & $939-1055$ & - \\
\hline & S\&G93 & $974-1072$ & - & $991-1045$ & - & $969-1098$ & - \\
\hline \multirow[t]{2}{*}{ Incl in $\mathrm{Cpx}$} & Cpx-melt, DN2001 & $1003-1017$ & - & $947-1000$ & - & - & - \\
\hline & S\&G93 & $1026-1049$ & - & $1030-1061$ & - & - & - \\
\hline Incl in Opx & S\&G93 & - & - & $1036-1045$ & - & - & - \\
\hline \multirow[t]{2}{*}{ Matrix Gl } & Ol-melt, DN2001 & 1030 & - & 1027 & - & $967-983$ & - \\
\hline & S\&G93 & 1097 & - & 1110 & - & $1106-1116$ & - \\
\hline \multirow[t]{3}{*}{ QUILF $_{\text {phen }}$} & $\mathrm{Sp}+\mathrm{Ol}+\mathrm{Cpx}$ & $912-978$ & $949 \pm 21$ & $912-939$ & $920 \pm 20$ & 909-976 & $951 \pm 23$ \\
\hline & $\mathrm{Sp}+\mathrm{Ol}+\mathrm{Opx}$ & - & - & $936-948$ & $942 \pm 28$ & - & - \\
\hline & $\mathrm{Cpx}-\mathrm{Opx}$ & 1095 & - & $1044-1124$ & $1080 \pm 38$ & - & - \\
\hline \multirow[t]{2}{*}{ QUILF $_{\text {grm }}$} & $\mathrm{Sp}+\mathrm{Ol}+\mathrm{Pig}+\mathrm{Cpx}$ & $1069-1178$ & $1116 \pm 54$ & $999-1009$ & $1002 \pm 4$ & - & - \\
\hline & $\mathrm{Sp}+\mathrm{Ol}+\mathrm{Pig}$ & - & - & - & - & $934-1138$ & $1022 \pm 78$ \\
\hline \multicolumn{8}{|c|}{ Pressure $(\mathrm{MPa})^{\mathrm{b}}$} \\
\hline Incl in $\mathrm{Ol}$ & Ol-Cpx-melt, DN96 & $0.1-330$ & $180 \pm 90$ & $0.1-230$ & $80 \pm 130$ & $0.1-330$ & $180 \pm 110$ \\
\hline Incl in Pl & O1-Cpx-melt, DN96 & $0.1-290$ & $80 \pm 100$ & $70-670$ & $220 \pm 170$ & $240-590$ & $380 \pm 100$ \\
\hline Incl in Cpx & Ol-Cpx-melt, DN96 & $80-190$ & $120 \pm 50$ & $0.1-180$ & $90 \pm 90$ & - & - \\
\hline \multicolumn{8}{|c|}{ Oxygen fugacity $(\Delta N N O)^{\mathrm{c}}$} \\
\hline Sp-melt & D\&S96 & $1.7-2.1$ & $1.9 \pm 0.1$ & $1.1-1.8$ & $1.3 \pm 0.1$ & $1.3-2.1$ & $1.6 \pm 0.2$ \\
\hline QUILF & $\mathrm{Sp}+\mathrm{Ol}+\mathrm{Opx}$ & $1.1-1.7$ & $1.4 \pm 0.1$ & $1.2-1.6$ & $1.3 \pm 0.1$ & $1.2-1.6$ & $1.5 \pm 0.1$ \\
\hline
\end{tabular}

${ }^{a}$ Temperatures calculated using empirical models of crystal-melt equilibria corrected for $\mathrm{H}_{2} \mathrm{O}$ in the melt using the program PETROLOG (Danyushevsky, 2001) and model of Sisson and Grove (1993b), with glass inclusion compositions corrected for post-entrapment host crystallization (Table 7); temperature calculations take the pressure estimates into account (b); Incl in Ol, Pl, Cpx=inclusions in olivine, plagioclase, clinopyroxene. QUILF temperatures calculated using the program QUILF (Andersen et al., 1993) using compositions of coexisting Ol, Opx, Cpx and $\mathrm{Fe}-\mathrm{Ti}$ oxides, and pressures of magma crystallization (subscripts phen=phenocrysts, grm=groundmass. References: DN2001=Danyushevsky (2001), S\&G93=Sisson and Grove (1993b), DN96=Danyushevsky et al. (1996), D\&S96=Danyushevsky and Sobolev (1996).

${ }^{b}$ Pressure of crystallization calculated from method of Danyushevsky et al. (1996) under assumption of multiple saturation of melt with O1 and Cpx; similar assumption was done for Pl-and Opx-hosted inclusions (see text).

${ }^{c}$ Oxygen fugacity (given in log unit deviation from NNO buffer) calculated from the compositions of spinel coexisting with melt (Sp-melt), QUILF=redox conditions calculated from olivine-orthopyroxene-magnetite equilibrium using the QUILF program.

partially lost $\mathrm{H}_{2} \mathrm{O}$, either from degassing of the magma or by $\mathrm{H}_{2} \mathrm{O}$ dissociation and loss of $\mathrm{H}$ after trapping, as suggested by Sobolev and Danyushevsky (1994). If this is true, comparison of the $\mathrm{H}_{2} \mathrm{O}-T$ relationships shown in Fig. 9b with water-independent temperature estimates can be used to reconstruct the original undegassed $\mathrm{H}_{2} \mathrm{O}$ contents in the magmas (see below).

The QUILF temperatures calculated from groundmass mineral compositions are considerably higher than those calculated from phenocryst data, although there is a quite large uncertainty (Table 9, Fig. 9a). This temperature difference is contrary to expectation if phenocrysts formed at an earlier stage of crystallization than the groundmass. We ascribe this apparent temperature increase to magma degassing and rapid crystallization at shallow depths. Loss of $\mathrm{H}_{2} \mathrm{O}$ will cause abundant crystallization of minerals because their liquidus temperatures are directly related to $\mathrm{H}_{2} \mathrm{O}$ contents in the melt. As a result of fast and abundant crystallization, the temperature in the degassing magma could rise locally due to the latent heat of crystallization.

\subsection{Pressure}

Pressures were estimated from the empirical method described in detail by Danyushevsky et al. (1996). 
The method employs the experimentally determined difference in $\mathrm{Ol}$ and $\mathrm{Cpx}$ temperatures of crystallization as a function of pressure, and has an estimated error of $\pm 250 \mathrm{MPa}$ at the $95 \%$ confidence level. The necessary condition for its use is multiple saturation of melt with olivine and clinopyroxene (i.e., cotectic crystallization). This is justified in our case by petrographic evidence for coexisting phenocrysts of $\mathrm{Ol}$, $\mathrm{Cpx}$ and $\mathrm{Pl}$ in the tephra. Also, because co-precipitation of plagioclase is indicated by the presence of $\mathrm{Pl}$ inclusions in both $\mathrm{Ol}$ and $\mathrm{Cpx}$ phenocrysts, we could use this approach to estimate crystallization pressure based on compositions of inclusions in $\mathrm{Pl}$ as well. The samples yielded a range of pressures from nearly atmospheric to a few hundred MPa (average 100$200 \mathrm{MPa}$ for the 1986 and 1853 eruptions and 200$400 \mathrm{MPa}$ for the prehistoric tephra; Table 9). Considering that these values are of the same order as the uncertainty of the method, we can conclude only that the pressure of crystallization was low. An independent pressure constraint was obtained by comparison of measured $\mathrm{H}_{2} \mathrm{O}$ and $\mathrm{Cl}$ contents in inclusion glasses with solubility isobars from Webster et al. (1999). As shown in Fig. 6c, the data suggest a maximum pressure of $200 \mathrm{Mpa}$ for $\mathrm{H}_{2} \mathrm{O}-\mathrm{Cl}$ solubility. Pressures can also be estimated from experimental $\mathrm{H}_{2} \mathrm{O}-\mathrm{CO}_{2}$ solubilities (Newman and Lowenstern, 2002 and references therein). Estimates are between 95 and 300 $\mathrm{MPa}$ if we assume that $\mathrm{H}_{2} \mathrm{O}$ was the dominant fluid component in equilibrium with melt containing 3.57.5 wt. $\% \mathrm{H}_{2} \mathrm{O}$. These are minimum estimates for the true crystallization pressures since $\mathrm{CO}_{2}$ is assumed to be negligible. Higher values for $\mathrm{CO}_{2}$ would shift the pressure to higher values as well.

\subsection{Redox conditions}

The redox conditions of magma crystallization were estimated using the equilibrium composition of spinel coexisting with melt after Danyushevsky and Sobolev (1996) and using the Ol-Opx-Mt equilbria incorporated in the QUILF program (Anderson et al., 1993). The oxygen fugacity values calculated by both methods agree within the uncertainties of the methods (approximately $\pm 0.6 \mathrm{log}$ units) and correspond to $\mathrm{NNO}+1$ to $\mathrm{NNO}+2$ for all samples studied (Table 9). These estimates are very close to those obtained from compositionally similar basalts from the Klyu- chevskoi volcano, Kamchatka, by Mironov et al. (2001) but are significantly higher than those proposed by Khubunaya and Sobolev (1998) for more primitive calc-alkaline magmas of the same volcano (FMQ).

\section{Heterogeneity of trapped melts as evidence for pre-eruption degassing}

Several lines of evidence suggest pre-eruptive degassing of $\mathrm{H}_{2} \mathrm{O}$ and $\mathrm{S}$ from the magma at the stage of phenocryst crystallization (trapping). First, there is the disparity between temperatures obtained by anhydrous mineral equilibria (QUILF) and meltmineral thermometry that depends on melt water contents (Fig. 9). If we equate the temperatures obtained from these two methods, we can estimate nondegassed $\mathrm{H}_{2} \mathrm{O}$ contents of the Chikurachki magmas at about 4-7 wt.\% $\mathrm{H}_{2} \mathrm{O}$, which corresponds to the upper range of values actually measured in melt inclusions. Similar high water concentrations (up to $6 \mathrm{wt} . \%$ $\mathrm{H}_{2} \mathrm{O}$ ) were reported from melt inclusion studies of other subduction-related basaltic to andesitic magmas (Izu-Bonin arc: Straub and Layne, 2003; Cerro Negro, Nicaragua: Roggensack et al., 1997). Second, the water and sulfur concentrations in primary glass inclusions extend over wide ranges, from high values reasonable for non-degassed subduction zone magmas based on other studies (e.g., Danyushevsky et al., 1993; Nilsson and Peach, 1993; Metrich and Clocchiatti, 1996; Sobolev and Chaussidon, 1996; Straub and Layne, 2003) to much lower values that approach the compositions of groundmass glass. If all degassing took place at eruption, one would expect more homogeneous melt inclusion compositions and a sharp drop in concentrations in the quenched groundmass glass that is not observed. Third, there is a range in An contents $\left(\mathrm{An}_{76-90}\right)$ of plagioclase inclusions in $\mathrm{Ol}$ and Cpx crystals that show no corresponding variations in their $m g$-number (Table 6). We interpret these variations in $\mathrm{Pl}$ composition to changes in $\mathrm{H}_{2} \mathrm{O}$ contents in the melt, implying some degassing took place during growth of the major phenocrysts. In principle, some of the $\mathrm{H}_{2} \mathrm{O}$ variations in glass inclusions could also be ascribed to post-entrapment loss of $\mathrm{H}_{2} \mathrm{O}$ due to dissociation and diffusion of $\mathrm{H}$ through the host mineral, as suggested by Sobolev and Danyushevsky (1994). We consider this process to be less important than 
partial degassing of $\mathrm{H}_{2} \mathrm{O}$ from the melt in our study because $\mathrm{H}_{2} \mathrm{O}$ dissociation and $\mathrm{H}$-loss produces oxidation of the inclusion glass, with the appearance of micrometer-sized magnetite crystals in Fe-rich melts. Some of the Chikurachki glass inclusions in Ol- and Cpx hosts do contain tiny magnetite crystals, suggesting loss of water by this mechanism. However, there are a number of inclusions with varying to low $\mathrm{H}_{2} \mathrm{O}$ contents that are composed of clear, fresh glass, and we interpret these inclusions as representing melts which have partially lost volatiles before trapping. The mechanism by which local volumes of melt can be partially degassed and trapped in growing phenocrysts to produce the heterogeneous suite of inclusions encountered in our samples is probably quite complex in detail. Most sulfur, like $\mathrm{H}_{2} \mathrm{O}$, is expected to exsolve at pressures as low as $20-30$ bars (corresponding to $<100-150 \mathrm{~m}$ of lithostatic thickness; e.g., Gerlach, 1986). It is therefore unlikely that significant amounts of sulfur and water could have been lost directly from the magma chamber under $80-380 \mathrm{MPa}$ crystallization pressure. We suggest a scenario involving vertical cycling of magma in pre-eruptive reservoir(s) that is similar to the magma drainback phenomenon described from Kilauea Volcano (e.g., Wallace and Anderson, 1998). Thus, the $\mathrm{H}_{2} \mathrm{O}-$ and S-poor inclusions may reflect melt domains that degassed as they ascended near the surface and were then entrained in downward flow as magmatic overpressure declined, to be mixed into fresher magmas at depth.

The possible implications of partial degassing for eruption style was discussed by Roggensack et al. (1997), among others, in their study of Cerro Negro volcano (Nicaragua). If magma degassing at shallow crustal depth is effective, eventual eruptions may be non-explosive, whereas rapid ascent of volatile-rich magma without the shallow degassing stage resulted in strong volatile release and highly explosive eruptions from the same volcano. The range of volatile contents displayed by melt inclusion populations can possibly indicate whether the erupted magma underwent an important stage of relatively passive degassing during phenocryst growth (mixed melt inclusion populations, variable $\mathrm{H}_{2} \mathrm{O}$ and $\mathrm{S}$ contents that approach groundmass values) or whether degassing was essentially a one-stage process linked to the eruption itself (homogeneous populations, sharp contrast to groundmass glass). It should be remem- bered that most of the historic eruptions from Chikurachki have been of the non-explosive character, possibly linked to episodes where volatiles were able to dissipate in a relatively shallow reservoir. Explosive eruptions, in contrast, occur when access to shallow levels is blocked or when the flux of magma from depth is too rapid for shallow degassing to prevent a catastrophic decompression of volatiles and explosive fragmentation of magma.

\section{Estimations of eruptive volatile release}

Using values for the total volumes of magma involved in the Chikurachki eruptions and the measured difference in volatile concentration between degassed groundmass glasses and the $\mathrm{H}_{2} \mathrm{O}$-rich melt inclusions, we can estimate the total mass of volatiles released during the eruptions. The total volumes of erupted magmas for the 1986 and 1853 events (dry-rock equivalents, DRE) are placed at 0.04 to $0.03 \mathrm{~km}^{3}$, respectively, taken from Belousov et al. (2003), and the resulting values for volatile emission $(E)$ were calculated from the following equation after Gerlach et al. (1994):

$E=10^{-3} \times \Delta_{\text {volatile }} \times \rho_{\text {melt }} \times \phi_{\text {melt }} \times V_{\text {melt }}$,

where $E=$ total volatile emission (the constant $10^{-3}$ gives $E$ in grams since $\Delta_{\text {volatile }}$ is in parts per million) of $\mathrm{H}_{2} \mathrm{O}, \mathrm{SO}_{2}$ and $\mathrm{Cl}, \Delta_{\text {volatile }}=$ in volatile content between glass inclusions and groundmass glasses (in parts per million); $\rho_{\text {melt }}=$ average density of the melt calculated from Lange and Carmichael (1987), in kg/ $\mathrm{km}^{3}, \phi_{\text {melt }}=$ melt volume fraction without crystals; $V_{\text {melt }}=$ the volume of erupted magma (in cubic kilometers DRE). We used estimates of $\Delta_{\text {volatile }}$ for water obtained from the maximum measured values of 5.5 and 4.8 wt. $\% \mathrm{H}_{2} \mathrm{O}$ in glass inclusions and in groundmass glasses from the 1986 and 1853 eruptions, respectively (minimum and maximum $\mathrm{H}_{2} \mathrm{O}$ limits in Table 10 take into account a 2-sigma standard error of \pm 1.2 wt. $\% \mathrm{H}_{2} \mathrm{O}$ ) and the $\mathrm{H}_{2} \mathrm{O}$ contents in the groundmass glasses. Our estimates for $\Delta_{\text {volatile }}$ for sulfur and chlorine are based on the measured groundmass glass values and a few glass inclusions (4 and 3 inclusions from the 1986 and 1853 eruptions, respectively; Table 10) having the highest concentrations of 
Table 10

Estimated syn-eruptive emissions of $\mathrm{H}_{2} \mathrm{O}, \mathrm{SO}_{2}$ and $\mathrm{Cl}$ during the 1986 and 1853 eruptions of the Chikurachki volcano

\begin{tabular}{|c|c|c|c|c|c|c|c|c|c|c|c|c|}
\hline \multirow[t]{2}{*}{ Sample } & \multirow{2}{*}{$\begin{array}{l}V_{\mathrm{DRE}} \\
\left(\mathrm{km}^{3}\right)\end{array}$} & \multirow{2}{*}{$\begin{array}{l}\rho_{\text {melt }} \\
\left(\mathrm{kg} / \mathrm{km}^{3}\right)\end{array}$} & \multirow[t]{2}{*}{$\phi_{\text {melt }}$} & \multirow{2}{*}{$\begin{array}{l}\mathrm{H}_{2} \mathrm{O} \\
\text { (wt.\%) }\end{array}$} & \multirow{2}{*}{$\begin{array}{l}\mathrm{S} \\
(\mathrm{ppm})\end{array}$} & \multirow{2}{*}{$\begin{array}{l}\mathrm{Cl} \\
(\mathrm{ppm})\end{array}$} & $\Delta \mathrm{H}_{2} \mathrm{O}$ & $\Delta \mathrm{S}$ & $\Delta \mathrm{Cl}$ & $\underline{E_{\mathrm{H} 2 \mathrm{O}}}$ & $E_{\mathrm{SO} 2}$ & $E_{\mathrm{Cl}}$ \\
\hline & & & & & & & \multicolumn{3}{|c|}{$\overline{\text { Melt volatile-loss (ppm) }}$} & \multicolumn{3}{|c|}{ Volatile emission $(\mathrm{g})$} \\
\hline The1986 eruption & 0.04 & $2.64 \mathrm{E}+12$ & 0.73 & - & - & - & & & & & & \\
\hline Glass inclusions $(N)^{\mathrm{a}}$ & & & & 1 & 4 & 30 & & & & & & \\
\hline Min & - & $2.59 \mathrm{E}+12$ & - & 4.3 & 1800 & 800 & 32,000 & 1450 & 0 & $2.5 \mathrm{E}+12$ & $2.2 \mathrm{E}+11$ & 0 \\
\hline Max & - & $2.72 \mathrm{E}+12$ & - & 6.7 & 2900 & 1200 & 56,000 & 2550 & 400 & $4.3 E+12$ & $3.9 \mathrm{E}+12$ & $3.1 \mathrm{E}+10$ \\
\hline Mean & - & $2.64 \mathrm{E}+12$ & - & 5.5 & 2200 & 900 & 44,000 & 1850 & 100 & $3.4 \mathrm{E}+12$ & $2.8 \mathrm{E}+11$ & $7.7 \mathrm{E}+09$ \\
\hline $1 \sigma$ error & - & - & - & 0.6 & 220 & 90 & 8500 & 230 & 120 & $9.4 \mathrm{E}+11$ & $6.7 \mathrm{E}+10$ & $9.4 \mathrm{E}+09$ \\
\hline Matrix Gl $(N=2)$ & - & $2.63 \mathrm{E}+12$ & - & 1.1 & 350 & 800 & & & & & & \\
\hline $1 \sigma$ error & - & - & - & 0.6 & 70 & 80 & & & & & & \\
\hline The1853 eruption & 0.03 & $2.59 \mathrm{E}+12$ & 0.77 & - & - & - & & & & & & \\
\hline Glass inclusions $(N)$ & & & & 1 & 3 & 22 & & & & & & \\
\hline Min & - & $2.54 \mathrm{E}+12$ & - & 3.6 & 1600 & 800 & 29,300 & 1250 & 0 & $1.8 \mathrm{E}+12$ & $1.5 \mathrm{E}+11$ & 0 \\
\hline Max & - & $2.70 \mathrm{E}+12$ & - & 6.0 & 2600 & 1300 & 53,300 & 2.250 & 500 & $3.2 \mathrm{E}+12$ & $2.7 \mathrm{E}+11$ & $3.0 \mathrm{E}+10$ \\
\hline Mean & - & $2.59 \mathrm{E}+12$ & - & 4.8 & 2000 & 1000 & 41,300 & 1650 & 200 & $2.5 \mathrm{E}+12$ & $2.0 \mathrm{E}+11$ & $1.2 \mathrm{E}+10$ \\
\hline $1 \sigma$ error & - & - & - & 2.0 & 200 & 100 & 8500 & 210 & 130 & $7.1 \mathrm{E}+11$ & $4.7 \mathrm{E}+10$ & $8.1 \mathrm{E}+09$ \\
\hline Matrix Gl $(N=4)$ & - & $2.59 \mathrm{E}+12$ & - & 0.7 & 350 & 800 & & & & & & \\
\hline $1 \sigma$ error & - & - & - & 0.6 & 70 & 80 & & & & & & \\
\hline \multicolumn{13}{|l|}{ Redoubt Volcano ${ }^{\mathrm{b}}$} \\
\hline Dec 14-22, 1989 & 0.015 & $2.3 \mathrm{E}+12$ & 0.75 & - & - & - & - & 750 & 800 & - & $3.9 \mathrm{E}+10$ & $2.1 \mathrm{E}+10$ \\
\hline Dome growth & 0.09 & $2.3 \mathrm{E}+12$ & 0.55 & - & - & - & - & 80 & 1000 & - & $1.8 \mathrm{E}+10$ & $1.1 \mathrm{E}+11$ \\
\hline \multicolumn{13}{|l|}{ Baitoushan Volcano ${ }^{\mathrm{b}}$} \\
\hline Comenditic rhyolite & 22 & $2.3 \mathrm{E}+12$ & 0.97 & - & - & - & 36,600 & 205 & 910 & $1.8 \mathrm{E}+15$ & $2.0 \mathrm{E}+12$ & $4.5 \mathrm{E}+13$ \\
\hline
\end{tabular}

$V_{\mathrm{DRE}}=$ volume (dense rock equivalent) of erupted magma (in cubic kilometers) from Belousov et al. (2003); $\rho_{\text {melt }}=$ melt density (in $\mathrm{kg} / \mathrm{km}^{3}$ ) calculated from matrix glass and melt inclusion compositions (Table 6) using the model of Lange and Carmichael (1987); $\phi_{\text {melt }}=$ melt volume fraction without crystals estimated from mass balance calculations using bulk-rock composition from Belousov et al. (2003), composition of matrix glasses (Table 6) and averaged compositions of Ol, Pl, Cpx and Opx phenocrysts; $-=$ no value.

$\Delta \mathrm{H}_{2} \mathrm{O}, \Delta \mathrm{S}$ and $\Delta \mathrm{Cl}$ (given in parts per million) represent melt volatile-loss calculated as the difference between melt inclusions and matrix glasses volatile contents (see text for details); the $1 \sigma$ error gives uncertainty of calculations defined from error propagation for independent random errors (Taylor, 1982).

$E_{\mathrm{H} 2 \mathrm{O}}, E_{\mathrm{SO} 2}$ and $E_{\mathrm{Cl}}$ are total emission values (given in grams) calculated from text Eq. (1) after Gerlach et al. (1994).

${ }^{\mathrm{a}} \mathrm{N}=$ number of inclusion values used to calculate minimum (Min), maximum (Max) and average (Mean) concentrations of volatiles in undegassed magma (see text for details).

${ }^{\mathrm{b}}$ Comparison of estimated total volatile emission calculated for Redoubt Volcano (Alaska) by Gerlach et al. (1994) and Baitoushan Volcano (China/North Korea) by Horn and Schmincke (2000) using the similar method.

sulfur and chlorine and also lying within the 4-sigma standard error range from the maximum values taken for a given eruption (done to account for $\mathrm{S}$ and $\mathrm{Cl}$ variations due to analytical uncertainty). The approach used by Gerlach et al. (1994) and in the present study assumes that there was no significant degassing of magma prior to eruption (probably incorrect for $\mathrm{H}_{2} \mathrm{O}$ and $\mathrm{S}$ as described above), so these estimates for syneruptive volatile loss must be considered as minima. The results (Table 10) indicate similar total volatile emissions for the 1986 and 1853 explosive eruptions of Chikurachki. Each of the eruptions released be- tween about 2000 and 3000 metric tons $( \pm 800)$ of $\mathrm{H}_{2} \mathrm{O}$, and 200 to 300 tons $( \pm 60)$ of $\mathrm{SO}_{2}$ into the atmosphere. The values for chlorine release $(8-12$ tons, \pm 9 ) are highly uncertain because of the only slight difference in $\mathrm{Cl}$ concentrations between glass inclusions and the groundmass glasses compared with the scatter within each group. Our estimated total uncertainties using error propagation (Taylor, 1982) for the terms in Eq. (1) are about $30 \%$ for $\mathrm{H}_{2} \mathrm{O}$ and $24 \%$ for $\mathrm{SO}_{2}$, but nearly $70 \%$ for $\mathrm{Cl}$ (Table 10 ). Some comparative estimates for total volalile release during explosive eruptions are given on Table 10, from which 
it can be seen that the Chikurachki eruptions released about ten times more $\mathrm{SO}_{2}$ and ten times less $\mathrm{Cl}$ than the 1989-1990 eruptions of Redoubt volcano (Gerlach et al., 1994), and significantly lower amounts of sulfur and chlorine than the plinian eruptions of rhyolite from Baitoushan volcano (Horn and Schmincke, 2000).

\section{Conclusions}

(1) The high concentrations of water (up to 6.4 wt.\%) determined by SIMS analysis of basaltic melt inclusions may be the cause for the highly explosive character of the Chikurachki 1986, 1853 and prehistoric plinian eruptions.

(2) Wide ranges of volatile contents in melt inclusions indicate heteogeneity of pre-aggregated melts in the Chikurachki magma system. The most $\mathrm{H}_{2} \mathrm{O}$ - and S-poor inclusions have compositions similar to degassed groundmass glass and probably represent melt fractions that were degassed near the summit reservoir and than drained back and mixed with undegassed or less degassed magmas at depth. The strombolian character of many eruptions from Chikurachki most probably reflect eruption of such degassed melts from shallow depths. This suggests the existence of periodically (during 100-200 year cycle) replenishing magma reservoir at depths of $>6 \mathrm{~km}$ beneath the Chikuracki volcano, which was able to supply compositionally similar volatile-rich magmas under similar conditions of pressure buildup and release to cause explosive style of eruptions.

(3) Crystallization of the Chikurachki water-rich basalt to basaltic andesite magmas occurred in the temperature range between 910 and $1180{ }^{\circ} \mathrm{C}$ at less than $400 \mathrm{MPa}$ total pressure and the redox conditions of $\mathrm{NNO}+1$ to $\mathrm{NNO}+2$. There is no substantial difference in pre-eruption conditions for the 1986, 1853 and prehistoric plinian eruption events.

(4) A comparison of volatile contents dissolved in glass inclusions and groundmass glasses permits minimum estimates of atmospheric loading during the 1986 and 1853 eruptions of
3400 and 2500 metric tons of $\mathrm{H}_{2} \mathrm{O}, 300$ and 200 tons of $\mathrm{SO}_{2}$, and 8 and 12 tons $\mathrm{Cl}$, respectively. These are about ten times higher for $\mathrm{SO}_{2}$ and ten times lower for $\mathrm{Cl}$ than for the 1989-1990 eruptions of Redoubt volcano (Gerlach et al., 1994).

\section{Acknowledgments}

We thank Marlina Elburg, Gerhard Worner, Maxim Portnyagin and Vadim Kamenetsky for fruitful discussions. Thorough reviews of three anonymous referees significantly improved this paper. The study was started during AAG's research stay in Potsdam supported by GFZ. Analytical work and manuscript preparation was completed at the MPI in Mainz, where AAG was supported by the Alexander von Humboldt Foundation (Wolfgang Paul Award to AVS). We acknowledge the Museum of Natural History, Washington DC, which provided us with the standards for electron microprobe analysis. Help and advice from Dieter Rhede, Oona Appelt, Michael Wiedenbeck and Elmar Groner during EPMA and SIMS analyses are very much appreciated.

\section{References}

Andersen, D.J., Lindsley, D.H., Davidson, P.M., 1993. QUILF: a PASCAL program to assess equilibria among $\mathrm{Fe}-\mathrm{Mg}-\mathrm{Ti}$ oxides, pyroxenes, olivine and quartz. Comput. Geosci. 19, $1333-1350$.

Arculus, R.J., 1994. Aspects of magma genesis in arcs. Lithos 33, 189-208.

Arculus, R.J., 2003. Use and abuse of the terms calcalkaline and calcalkalic. J. Petrol. 44, 929-935.

Ariskin, A.A., Frenkel, M.Ya., Barmina, G.S., Nielsen, R.L., 1993. COMAGMAT: a FORTRAN program to model magma differentiation processes. Comput. Geosci. 19, 1155-1170.

Ayers, J.C., Dittmer, S.K., Layne, G., 1997. Partitioning of elements between peridotite and $\mathrm{H}_{2} \mathrm{O}$ at $2.0\left(3.0 \mathrm{GPa}\right.$ and $900-1100{ }^{\circ} \mathrm{C}$, and application to models of subduction zone processes. Earth Planet. Sci. Lett. 150, 381-398.

Azough, F., Freer, R., 2000. Iron diffusion in single-crystal diopside. Phys. Chem. Miner. 27, 732-740.

Bailey, J.C., Frolova, T.I., Burikova, I.A., 1987. Mineralogy, geochemistry and petrogenesis of Kurile Island Arc basalts. Contrib. Mineral. Petrol. 102, 265-280.

Bailey, J.C., Larsen, O., Frolova, T.I., 1987. Strontium isotope variations in lower Tertiary-Quaternary volcanic rocks from the Kurile Island Arc. Contrib. Mineral. Petrol. 95, 155-165. 
Belousov, A.B., Belousova, M.G., Grushin, S.Yu., Krestov, P.B., 2003. Historic eruptions of the Chikurachki volcano (Paramushir, Kurile Islands). Volcanol. Seismol. 3, 15-34 (in Russian).

Borisov, A.A., Shapkin, A.I., 1990. A new empirical equation rating $\mathrm{Fe}^{3+} / \mathrm{Fe}^{2+}$ in magmas to their composition, oxygen fugacity, and temperature. Geochem. Int. 27, 111-116.

Brasseur, G., Granier, C., 1992. Mount Pinatubo aerosols, chlorofluorocarbons and ozone depletion. Science 257, 1239-1242.

Brenan, J.M., Shaw, H.F., Ryerson, F.J., Phinney, D.L., 1995. Mineral-aqueous fluid partitioning of trace elements at $900{ }^{\circ} \mathrm{C}$ and $2.0 \mathrm{GPa}$ : constraints on the trace element chemistry of mantle and deep crustal fluids. Geochim. Cosmochim. Acta $59,3331-3350$.

Carey, S., Sparks, R.S.J., 1986. Quantitative models of the fallout and dispersal of tephra from volcanic eruption columns. Bull. Volcanol. 48, 109-125.

Carroll, M.R., Webster, J.D., 1994. Solubilities of sulfur, noble gases, nitrogen, chlorine, and fluorine in magmas. In: Carroll, M.R., Holloway, J.R. (Eds.), Volatiles in Magmas, Rev. Mineral., vol. 30. Mineral. Soc. Am., Washington, D.C, pp. 231-279.

Chakraborty, S., 1997. Rates and mechanisms of Fe-Mg interdiffusion in olivine at $980-1300{ }^{\circ} \mathrm{C}$. J. Geophys. Res. 102, $12317-12331$

Chaussidon, M., Libourel, G., 1993. Boron partitioning in the upper mantle: an experimental and ion probe study. Geochim. Cosmochim. Acta 57, 5053-5062.

Chaussidon, M., Jambon, A., 1994. Boron content and isotopic composition of oceanic basalts: geochemical and cosmochemical implications. Earth Planet. Sci. Lett. 121, 277-291.

Danyushevsky, L.V., 2001. The effect of small amounts of $\mathrm{H}_{2} \mathrm{O}$ on crystallization of mid-ocean ridge and backarc basin magmas. J. Volcanol. Geotherm. Res. 110, 265-280.

Danyushevsky, L.V., Sobolev, A.V., 1996. Ferric-ferrous ratio and oxygen fugacity calculations for primitive mantle-derived melts: calibration of an empirical technique. Mineral. Petrol. 57, 229-241.

Danyushevsky, L.V., Falloon, T.J., Sobolev, A.V., Crawford, A.J., Carroll, M., Price, R.C., 1993. The $\mathrm{H}_{2} \mathrm{O}$ content of basalt glasses from Southwest Pacific back-arc basins. Earth Planet. Sci. Lett. $117,347-362$

Danyushevsky, L.V., Sobolev, A.V., Dmitriev, L.V., 1996. Estimation of the pressure of crystallization and $\mathrm{H}_{2} \mathrm{O}$ content of MORB and BABB glasses: calibration of an empirical technique. Mineral. Petrol. 57, 185-204.

Danyushevsky, L.V., Della-Pasqua, F.N., Sokolov, S., 2000. Reequilibration of melt inclusions trapped by magnesian olivine phenocrysts from subduction-related magmas: petrological implications. Contrib. Mineral. Petrol. 138, 68-83.

Devine, J.D., Gardner, J.E., Brack, H.P., Layne, G.D., Rutherford, M.J., 1995. Comparison of microanalytical methods for estimating $\mathrm{H}_{2} \mathrm{O}$ contents of silicic volcanic glasses. Am. Mineral. 80, 319-328.

Dimanov, A., Sautter, V., 2000. "Average" interdiffusion of (Fe, $\mathrm{Mn})-\mathrm{Mg}$ in natural diopside. Eur. J. Mineral. 12, 749-760.

Dixon, J.E., Clague, D.A., Stolper, E.M., 1991. Degassing history of water, sulfur and carbon in submarine lavas from Kilauea volcano, Hawaii. J. Geol. 99, 371-394.
Fahey, A.J., Zinner, E.K., Crozaz, G., Kornacki, A.S., 1987. Microdistributions of $\mathrm{Mg}$ isotopes and REE abundances in a Type A calcium-aluminum-rich inclusion from Efremovka. Geochim. Cosmochim. Acta 51, 3215-3229.

Fedotov, S.A., Markhinin, Ye.K., 1983. The Great Tolbachik Fissure Eruption. Cambridge Univ. Press, New York.

Ford, C.E., Russel, D.G., Craven, J.A., Fisk, M.R., 1983. Olivineliquid equilibria: temperature, pressure and composition dependence of the crystal/liquid cation partition coefficients for $\mathrm{Mg}$, $\mathrm{Fe}^{2+}, \mathrm{Ca}$ and Mn. J. Petrol. 24, 256-265.

Gerlach, T.M., 1986. Exsolution of $\mathrm{H}_{2} \mathrm{O}, \mathrm{CO}_{2}$, and $\mathrm{S}$ during eruptive episodes at Kilauea Volcano, Hawaii. J. Geophys. Res. 91, $12177-12185$.

Gerlach, T.M., Westrich, H.R., Casadevall, T.J., Finnegan, D.L., 1994. Vapor saturation and accumulation in magmas of the 1989-1990 eruption of Redoubt Volcano, Alaska. J. Volcanol. Geotherm. Res. 62, 317-337.

Gill, J., Torssander, P., Lapierre, H., Taylor, R., Kaiho, K., Koyama, M., Kusakabe, M., Aitchison, J., Cisowski, S., Dadey, K., Fujioka, K., Klaus, A., Lovell, M., Marsaglia, K., Pezard, P., Taylor, B., Tazaki, K., 1990. Explosive deep-water basalt in the Sumisu Backarc Rift. Science 248, 1214-1217.

Gladney, E.S., Roelandts, I., 1987. Compilation of boron concentration data for NBS, USG and CCRMP reference materials. Geostand. Newsl. 11, 167-185.

Gorshkov, G.S., 1967. Volcanism of the Kurile Island Arc. Nauka, Moscow. (in Russian).

Grove, T.L., Elkins-Tanton, L.T., Parman, S.W., Chatterjee, N., Müntener, O., Gaetani, G.A., 2003. Fractional crystallization and mantle-melting controls on calc-alkaline differentiation trends. Contrib. Mineral. Petrol. 145, 515-533.

Gurenko, A.A., Schmincke, H.-U., 2002. Orthopyroxene-bearing tholeiites of the Iblean Plateau (Sicily): constraints on magma origin and evolution from glass inclusions in olivine and orthopyroxene. Chem. Geol. 183, 305-331.

Gurenko, A.A., Sobolev, A.V., Kononkova, N.N., 1991. Petrology of the primary magma of the Reykjanes Peninsula rift tholeiites. Geochem. Int. 28, 58-71.

Gurenko, A.A., Hansteen, T.H., Schmincke, H.-U., 1998. Melt, crystal, and fluid inclusions in olivine and clinopyroxene phenocrysts from the submarine shield stage hyaloclastites of Gran Canaria, Sites 953 and 956. In: Weaver, P.P.E., Schmincke, H.U., Firth, J.V., Duffield, W. (Eds.), Proceedings of the Ocean Drilling Program, Scientific Results. College Station, TX (Ocean Drilling Program), vol. 157, pp. 375-401.

Hofmann, A.W., 1988. Chemical differentiation of the Earth: the relationship between mantle, continental crust and oceanic crust. Earth Planet. Sci. Lett. 90, 297-314.

Holtz, F., Berhens, H., Dingwell, D.B., Taylor, R.P., 1992. Water solubility in aluminosilicate melts of haplogranite composition at 2 kbar. Chem. Geol. 96, 289-302.

Holtz, F., Johannes, W., Tamic, N., Behrens, H., 2001. Maximum and minimum water contents of granitic melts generated in the crust: a reevaluation and implications. Lithos 56, 1-14.

Horn, S., Schmincke, H.-U., 2000. Volatile emission during the eruption of Baitoushan Volcano (China/North Korea) ca. 969 AD. Bull. Volcanol. 61, 537-555. 
Houghton, B.F., Wilson, C.J.N., Del Carlo, P., Coltelli, M., Sable, J.E., Carey, R., 2004. The influence of conduit processes on changes in style of basaltic Plinian eruptions: Tarawera 1886 and Etna 122 BC. J. Volcanol. Geotherm. Res. 137, 1-14.

Ikeda, Y., 1998. Geochemistry of Miocene back-arc basin basalts from northeast Hokkaido, Japan. J. Geol. Soc. Jpn. 104, 99-106.

Ikeda, Y., Stern, R.J., Kagami, H., Sun, C.-H., 2000. Pb, Nd, and Sr isotopic constraints on the origin of Miocene basaltic rocks from northeast Hokkaido, Japan: implications for opening of the Kurile back-arc basin. Isl. Arc 9, 161-172.

Ishikawa, T., Tera, F., 1997. Source, composition and distribution of the fluid in the Kurile mantle wedge: constraints from across-arc variations of $\mathrm{B} / \mathrm{Nb}$ and $\mathrm{B}$ isotopes. Earth Planet. Sci. Lett. 152, $123-138$.

Jarosewich, E.J., Nelen, J.A., Norberg, J.A., 1980. Reference samples for electron microprobe analyses. Geostand. Newsl., J. Geostand. Geoanalys. 4, 43-47.

Jochum, K.P., Dingwell, D.B., Rocholl, A., Stoll, B., Hofmann, A.W., Becker, S., Besmehn, A., Bessette, D., Dietze, H.-J., Dulski, P., Erzinger, J., Hellebrand, E., Hoppe, P., Horn, I., Janssens, K., Jenner, G.A., Klein, M., McDonough, W.F., Maetz, M., Mezger, K., Münker, C., Nikogosian, I.K., Pickhardt, C., Raczek, I., Rhede, D., Seufert, H.M., Simakin, S.G., Sobolev, A.V., Spettel, B., Straub, S., Vincze, L., Wallianos, A., Weckwerth, G., Weyer, S., Wolf, D., Zimmer, M., 2000. The preparation and preliminary characterization of eight geological MPI-DING reference glasses for in-situ microanalysis. Geostand. Newsl., J. Geostand. Geoanalys. 24, $87-133$.

Kamenetsky, V.S., Everard, J.L., Crawford, A.J., Varne, R., Eggins, S.M., Lanyon, R., 2000. Enriched end-member of primitive MORB melts: petrology and geochemistry of glasses from Macquarie Island (SW Pacific). J. Petrol. 41, 411-430.

Kersting, A.B., Arculus, R.J., 1994. Klyuchevskoy volcano, Kamchatka, Russia: the role of high-flux recharged, tapped, and fractionated magma chamber(s) in the genesis of high- $\mathrm{Al}_{2} \mathrm{O}_{3}$ from high-MgO basalt. J. Petrol. 35, 1-41.

Khubunaya, S.A., Sobolev, A.V., 1998. Primary melts of calcalkaline magnesian basalts from the Klyuchevskoi volcano, Kamchatka. Dokl. Earth Sci. 360, 537-539.

Lange, R.A., Carmichael, I.S.E., 1987. Densities of $\mathrm{Na}_{2} \mathrm{O}-\mathrm{K}_{2} \mathrm{O}-$ $\mathrm{CaO}-\mathrm{MgO}-\mathrm{FeO}-\mathrm{Fe}_{2} \mathrm{O}_{3}-\mathrm{Al}_{2} \mathrm{O}_{3}-\mathrm{TiO}_{2}-\mathrm{SiO}_{2}$ liquids: new measurements and derived partial molar properties. Geochim. Cosmochim. Acta 53, 2195-2204.

Mathez, E.A., 1976. Sulfur solubility and magmatic sulfides in submarine basalt glasses. J. Geophys. Res. 81, 4269-4276.

Maurel, C., Maurel, P., 1982. Etude expérimentale de l'equilibre $\mathrm{Fe}^{2+}-\mathrm{Fe}^{3+}$ dans les spinelles chromifères et les liquides silicatés basiques coexistants, à 1 atm. C.R. Acad. Sci. Paris 295, $209-212$.

Metrich, N., Rutherford, M.J., 1992. Experimental study of chlorine behavior in hydrous silicic melts. Geochim. Cosmochim. Acta $56,60 \tilde{7} 616$.

Metrich, N., Clocchiatti, R., 1996. Sulfur abundance and its speciation in oxidized alkaline melts. Geochim. Cosmochim. Acta $60,415 \tilde{1} 4160$.
Mironov, N.L., Portnyagin, M.V., Plechov, P.Yu., Khubunaya, S.A., 2001. Late evolution stages of magmas of the Klyuchevskoi volcano (Kamchatka) based on the results of study of melt inclusions in minerals from high-alumina basalts. Petrology 9, 51-69 (in Russian).

Miyashiro, A., 1974. Volcanic rock series in Isl. Arcs and active continental margins. Am. J. Sci. 274, 321-355.

Mosbah, M., Metrich, N., Massiot, P., 1991. PIGME fluorine determination using a nuclear microprobe with application to glass inclusions. Nucl. Instrum. Methods Phys. Res., B Beam Interact. Mater. Atoms 58, 227-231.

Newman, S., Lowenstern, J.B., 2002. VolatileCalc: a silicate melt$\mathrm{H}_{2} \mathrm{O}-\mathrm{CO}_{2}$ solution model written in Visual Basic for Excel. Comput. Geosci. 28, 597-604.

Nilsson, K., Peach, C.L., 1993. Sulfur speciation, oxidation state, and sulfur concentration in backarc magmas. Geochim. Cosmochim. Acta 57, $380 \tilde{7} 3813$.

Ovsyannikov, A.A., Muravyev, Y.D., 1992. Eruptions of the Chikurachki volcano. Volcanol. Seismol. 5-6, 3-21 (in Russian).

Pearce, J.A., Peate, D.W., 1995. Tectonic implications of the composition of volcanic arc magmas. Annu. Rev. Earth Plan. Sci. 24, 251-285.

Pearce, N.J.G., Perkins, W.T., Westgate, J.A., Gorton, M.P., Jackson, S.E., Neal, C.R., Chenery, S.P., 1997. A compilation of new and published major and trace element data for NIST SRM 610 and SRM 612 partially certified glass reference materials. Geostand. Newsl. 21, 115-144.

Pichavant, M., 1987. Effects of $\mathrm{B}$ and $\mathrm{H}_{2} \mathrm{O}$ on liquidus phase relations in the haplogranite system at $1 \mathrm{kbar}$. Am. Mineral. 72, 1056-1070.

Rautenschlein, M., Jenner, G.A., Hertogen, J., Hofmann, A.W., Kerrich, R., Schmincke, H.-U., White, W.M., 1985. Isotopic and trace element composition of volcanic glasses from the Akaki Canyon, Cyprus: implications for the origin of the Troodos ophiolite. Earth Planet. Sci. Lett. 75, 369-383.

Robin, C., Eissen, J.P., Monzier, M., 1993. Giant tuff cone and 12km-wide associated caldera at Ambrym Volcano (Vanuatu, New Hebrides Arc). J. Volcanol. Geotherm. Res. 55, 225-238.

Roggensack, K., Hervig, R.L., McKnight, S.B., Williams, S.N., 1997. Explosive basaltic volcanism from Cerro Negro volcano: influence of volatiles on eruptive style. Science 277, $1639-1642$.

Ryan, J.G., Morris, J., Tera, F., Leeman, W.P., Tsvetkov, A., 1995. Cross-arc geochemical variations in the Kurile Arc as a function of slab depth. Science 270, 625-627.

Signorelli, S., Carroll, M.R., 2001. Experimental constraints on the origin of chlorine emissions at the Soufrière Hills volcano, Montserrat. Bull. Volcanol. 62, 431-440.

Sisson, T.W., Grove, T.L., 1993a. Experimental investigations of the role of $\mathrm{H}_{2} \mathrm{O}$ in calc-alkaline differentiation and subduction zone magmatism. Contrib. Mineral. Petrol. 113, 143-166.

Sisson, T.W., Grove, T.L., 1993b. Temperatures and $\mathrm{H}_{2} \mathrm{O}$ contents of low-MgO high-alumina basalts. Contrib. Mineral. Petrol. $113,167-184$.

Sobolev, A.V., 1983. Origin of Siberian Meimechites in relation to the general problem of ultramafic magma. Ph. D. thesis, Vernadsky Inst. of Geochemistry Moscow, USSR (in Russian). 
Sobolev, A.V., Danyushevsky, L.V., 1994. Petrology and geochemistry of boninites from the north termination of the Tonga Trench: constraints on the generation conditions of primary high-Ca boninite magmas. J. Petrol. 35, 1183-1213.

Sobolev, A.V., Chaussidon, M., 1996. $\mathrm{H}_{2} \mathrm{O}$ concentrations in primary melts from supra-subduction zones and mid-ocean ridges: implications for $\mathrm{H}_{2} \mathrm{O}$ storage and recycling in the mantle. Earth Planet. Sci. Lett. 137, 45-55.

Sobolev, A.V., Portnyagin, M.V., Dmitriev, L.V., Tsameryan, O.P., Danyushevsky, L.V., Kononkova, N.N., Shimizu, N., Robinson, P.T., 1993. Petrology of ultramafic lavas and associated rocks of the Troodos massif, Cyprus. Petrology 1, 331-361.

Stalder, R., Foley, S.F., Brey, G.P., Horn, I., 1998. Mineral-aqueous fluid partitioning of trace elements at $900-1200{ }^{\circ} \mathrm{C}$ and $3.0-5.7$ $\mathrm{GPa}$ : new experimental data for garnet, clinopyroxene, and rutile, and implications for mantle metasomatism. Geochim. Cosmochim. Acta 62, 1781-1801.

Stix, J., Layne, G.D., 1996. Gas saturation and evolution of volatile and light lithophile elements in the Bandelier magma chamber between two caldera-forming eruptions. J. Geophys. Res. 101, $25181-25196$

Straub, S.M., Layne, G.D., 2003. The systematics of chlorine, fluorine, and water in Izu arc front volcanic rocks: implications for volatile recycling in subduction zones. Geochim. Cosmochim. Acta 67, 4179-4203.

Takagi, T., Orihashi, Y., Naito, K., Watanabe, Y., 1999. Petrology of a mantle-derived rhyolite, Hokkaido, Japan. Chem. Geol. 160, $425-445$.

Tatsumi, Y., Eggins, S., 1995. Subduction Zone Magmatism. Blackwell, Cambridge.

Tatsumi, Y., Furukawa, Y., Kogiso, T., Yamada, Y., Yokoyama, T., Fedotov, S.A., 1994. A third volcanic chain in Kamchatka: thermal anomaly at transform/convergence plate boundary. Geophys. Res. Lett. 21, 537-540.
Taylor, J.R., 1982. An Introduction to Error Analysis. Oxford Univ. Press, Oxford.

Thordarson, T., Self, S., Oskarsson, N., Hulsebosch, T., 1996. Sulfur, chlorine, and fluorine degassing and atmospheric loading by the 1783-1784 AD Laki (Skaftár Fires) eruption in Iceland. Bull. Volcanol. 58, 205-225.

Walker, G.P.L., 1973. Explosive volcanic eruptions - a new classification scheme. Geol. Rundsch. 62, 431-446.

Walker, G.P.L., 1980. The Taupo pumice: product of the most powerful known (ultraplinian) eruption? J. Volcanol. Geotherm. Res. 8, 69-94.

Wallace, P., Carmichael, I.S.E., 1992. Sulfur in basaltic magmas. Geochim. Cosmochim. Acta 56, 1863-1874.

Wallace, P.J., Carmichael, I.S.E., 1994. S speciation in submarine basaltic glasses as determined by measurements of $\mathrm{SK}_{\alpha} \mathrm{X}$-ray wavelength shifts. Am. Mineral. 79, 1611167.

Wallace, P.J., Anderson, A.T.J., 1998. Effects of eruption and lava drainback on the $\mathrm{H}_{2} \mathrm{O}$ contents of basaltic magmas at Kilauea Volcano. Bull. Volcanol. 59, 327-344.

Webster, J.D., 1992. Fluid-melt interactions involving Cl-rich granites: experimental study from 2 to 8 kbar. Geochim. Cosmochim. Acta. 56, 659̃678.

Webster, J.D., Kinzler, R.J., Mathez, E.A., 1999. Chloride and water solubility in basalt and andesite melts and implications for magmatic degassing. Geochim. Cosmochim. Acta 63, 729-738.

Williams, S.N., 1983. Plinian airfall deposits of basaltic composition. Geology 11, 211-214.

Wilson, L., Walker, G.P.L., 1987. Explosive volcanic eruptions VI. Ejecta dispersal in plinian eruptions: the control of eruption conditions and atmospheric properties. Geophys. J. R. Astron. Soc. 89, 657-679.

Zinner, E., Crozaz, G., 1986. A method for the quantitative measurement of rare earth elements in the ion microprobe. Int. J. Mass Spectrom. Ion Process. 69, 17-38. 